\title{
Tandem Incorporation of Enantiomeric Residues Engenders Discrete Peptoid Structures
}

\author{
Benjamin C. Gorske*, Emily M. Mumford, and Rebecca R. Conry. \\ Department of Chemistry, Bowdoin College, 6600 College Station, \\ Brunswick, ME 04011-8466 \\ E-mail:bgorske@bowdoin.edu
}

Supporting Information.

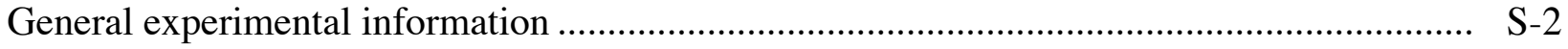

Peptoid synthesis and characterization .................................................................... S-3

High-resolution mass spectrometry data ............................................................ S-4

Analytical RP-HPLC data .......................................................................................... S-5

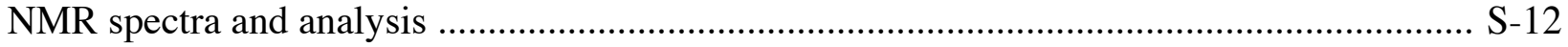

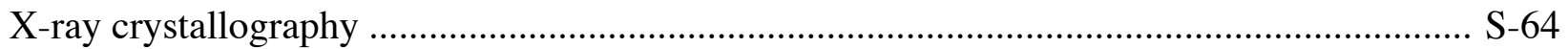

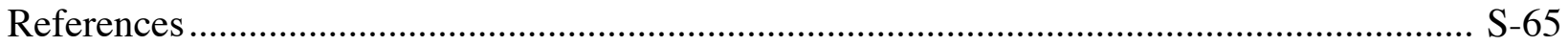

${ }^{*}$ Corresponding author. 
General. Unless otherwise noted, all reagents were 99\% purity, ACS-grade or HPLC-grade, and obtained from Sigma-Aldrich Chemicals (St. Louis, MO). Solvents were purchased from Fisher Scientific (Fair Lawn, New Jersey) and were not subjected to distillation. Thin layer chromatography (TLC) was performed on glass silica gel plates (EMD Chemicals, Inc. Gibbstown, NJ), 250 micron, with UV254. Silica gel chromatography was performed with silica gel from Acros Organics (New Jersey). TLC plates were visualized using an ultraviolet lamp and heat-activated staining with potassium permanganate, ceric ammonium molybdate, and $p$ anisaldehyde.

Instrumentation. All ${ }^{1} \mathrm{H}(400 \mathrm{MHz})$ Nuclear Magnetic Resonance (NMR) spectra were collected on a Bruker $400 \mathrm{MHz}$ DPX NMR Spectrometer. All samples were prepared as $\sim 25 \mathrm{mM}$ solutions in $\mathrm{CDCl}_{3}$, unless otherwise noted. Chemical shifts were recorded in parts per million (ppm), using tetramethyl silane (TMS) as the $0.0 \mathrm{ppm}$ reference, unless otherwise noted. Twodimensional NMR spectra were analyzed using Sparky. ${ }^{1}$

High-resolution mass spectrometric analysis was performed using a 6530 quadrupole time-of-flight (Q-TOF) mass analyzer (Agilent Technologies, Santa Clara CA), equipped with a 1260 Chip Cube nano-electrospray ionization (nanoESI) source. Chromatographic separation and nanoESI was performed using a ProtID-chip with a $40 \mathrm{~nL}$ enrichment column and a $43 \mathrm{~mm}$ x $75 \mu \mathrm{m}$ analytical column (Agilent Technologies), both packed with $300 \AA$, $5 \mu \mathrm{m}$ particles with C18 stationary phase. The mobile phases were $0.1 \%$ formic acid $/ \mathrm{H}_{2} \mathrm{O}(\mathrm{A})$ and $0.1 \%$ formic acid/acetonitrile (B). Samples were loaded using 98:2 (A:B) and eluted using a linear gradient with 30:70 (A:B) at 12 min. Mass spectra were collected in positive ion mode; spectra were internally calibrated using methyl stearate $\left(\mathrm{C}_{17} \mathrm{H}_{35} \mathrm{CO}_{2} \mathrm{CH}_{3}\right)$ and hexakis $(1 \mathrm{H}, 1 \mathrm{H}, 4 \mathrm{H}-$ hexafluorobutyloxy)phosphazine (HP-1221; $\mathrm{C}_{24} \mathrm{H}_{18} \mathrm{O}_{6} \mathrm{~N}_{3} \mathrm{P}_{3} \mathrm{~F}_{36}$ ), continuously introduced and 
detected as $[\mathrm{M}+\mathrm{H}]^{+}$.

Analytical reversed phase high performance liquid chromatography (RP-HPLC) was used to assess the purity of peptoid products. Samples were prepared in acetonitrile and loaded onto an Agilent 1100 HPLC system (Agilent Technologies) equipped with a Vydac C18 monomeric column (Grace, Deerfield, IL) and a UV absorbance detector (Agilent, Santa Clara, CA). Water containing $0.1 \%$ TFA (solvent A; Fisher-Scientific) and methanol containing $0.1 \%$ TFA (solvent B; Fisher-Scientific) were employed as the two mobile phase components. Unless otherwise noted, products were analyzed with one of two gradient elutions at $2.0 \mathrm{ml} / \mathrm{min}$ : Method 1 (95\% A $/ 5 \%$ B for $2 \min , 95 \%$ A $/ 5 \%$ B to $100 \%$ B over 6 min, $100 \%$ B for 7 min) or Method $2(50 \%$ $\mathrm{A} / 50 \% \mathrm{~B}$ for $2 \mathrm{~min}, 50 \% \mathrm{~A} / 50 \% \mathrm{~B}$ to $100 \%$ B over $5 \mathrm{~min}, 100 \% \mathrm{~B}$ for $11 \mathrm{~min}$ ).

Preparative HPLC was used to purify the peptoids and was performed using an Agilent 1100 HPLC system equipped with an Agilent Zorbex SB-C18 column (Agilent Technologies). The crude peptoids were dissolved in acetonitrile, and methanol containing 0.1\% TFA (FisherScientific) was employed as the mobile phase for the isocratic separations at a flow rate of 5.0 $\mathrm{mL} / \mathrm{min}$. The solvent was removed from the combined fractions in vacuo and the residue was placed under high vacuum prior to analysis.

Peptoid synthesis and characterization. All peptoids were synthesized according to previously reported procedures ${ }^{2,3}$ All compounds were purified to $>95 \%$ homogeneity by RP-HPLC prior to analytical HPLC, NMR, and high-resolution mass spectrometric analysis. Full characterization and proof-of-purity data for each peptoid are provided below. 
High-resolution mass spectrometry data.

Table S-1. Calculated and observed masses for peptoids 1-14. .

\begin{tabular}{ccc}
\hline compound & calculated mass & observed mass \\
$\mathbf{1}$ & 539.2904 & $539.2901[\mathrm{M}+\mathrm{H}]^{+}$ \\
$\mathbf{2}$ & 750.3901 & $750.3835[\mathrm{M}+\mathrm{H}]^{+}$ \\
$\mathbf{3}$ & 961.4899 & $961.4899[\mathrm{M}+\mathrm{H}]^{+}$ \\
$\mathbf{4}$ & 1172.5896 & $1172.5800[\mathrm{M}+\mathrm{H}]^{+}$ \\
$\mathbf{5}$ & 721.3748 & $721.3767[\mathrm{M}+\mathrm{H}]^{+}$ \\
$\mathbf{6}$ & 779.4167 & $779.4069[\mathrm{M}+\mathrm{H}]^{+}$ \\
$\mathbf{7}$ & 425.2224 & $425.2282[\mathrm{M}+\mathrm{H}]^{+}$ \\
$\mathbf{8}$ & 636.3221 & $636.3256[\mathrm{M}+\mathrm{H}]^{+}$ \\
$\mathbf{9}$ & 847.4218 & $847.4221[\mathrm{M}+\mathrm{H}]^{+}$ \\
$\mathbf{1 0}$ & 1058.5216 & $1058.5209[\mathrm{M}+\mathrm{H}]^{+}$ \\
$\mathbf{1 1}$ & 486.2751 & $486.2830[\mathrm{M}+\mathrm{H}]^{+}$ \\
$\mathbf{1 2}$ & 847.4218 & $847.4208[\mathrm{M}+\mathrm{H}]^{+}$ \\
$\mathbf{1 3}$ & 905.4636 & $905.4693[\mathrm{M}+\mathrm{H}]^{+}$ \\
$\mathbf{1 4}$ & 1116.5634 & $1116.5577[\mathrm{M}+\mathrm{H}]^{+}$ \\
& & \\
\hline
\end{tabular}




\section{Analytical RP-HPLC data (220 nm)}

1: Ac-r1npe-s1npe-OtBu (Method 1)

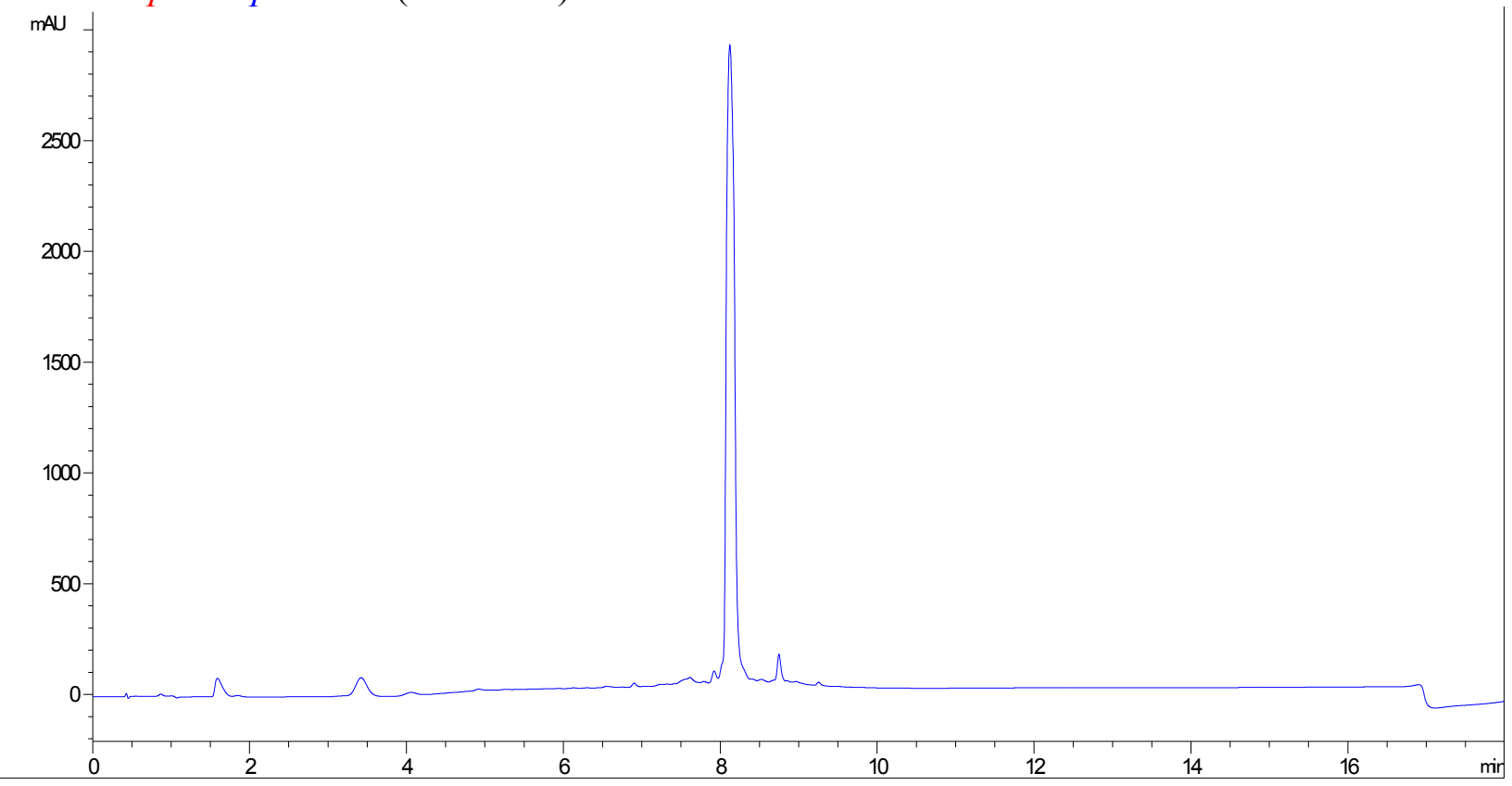

2: Ac-s1npe-r1npe-s1npe-OtBu (Method 2)

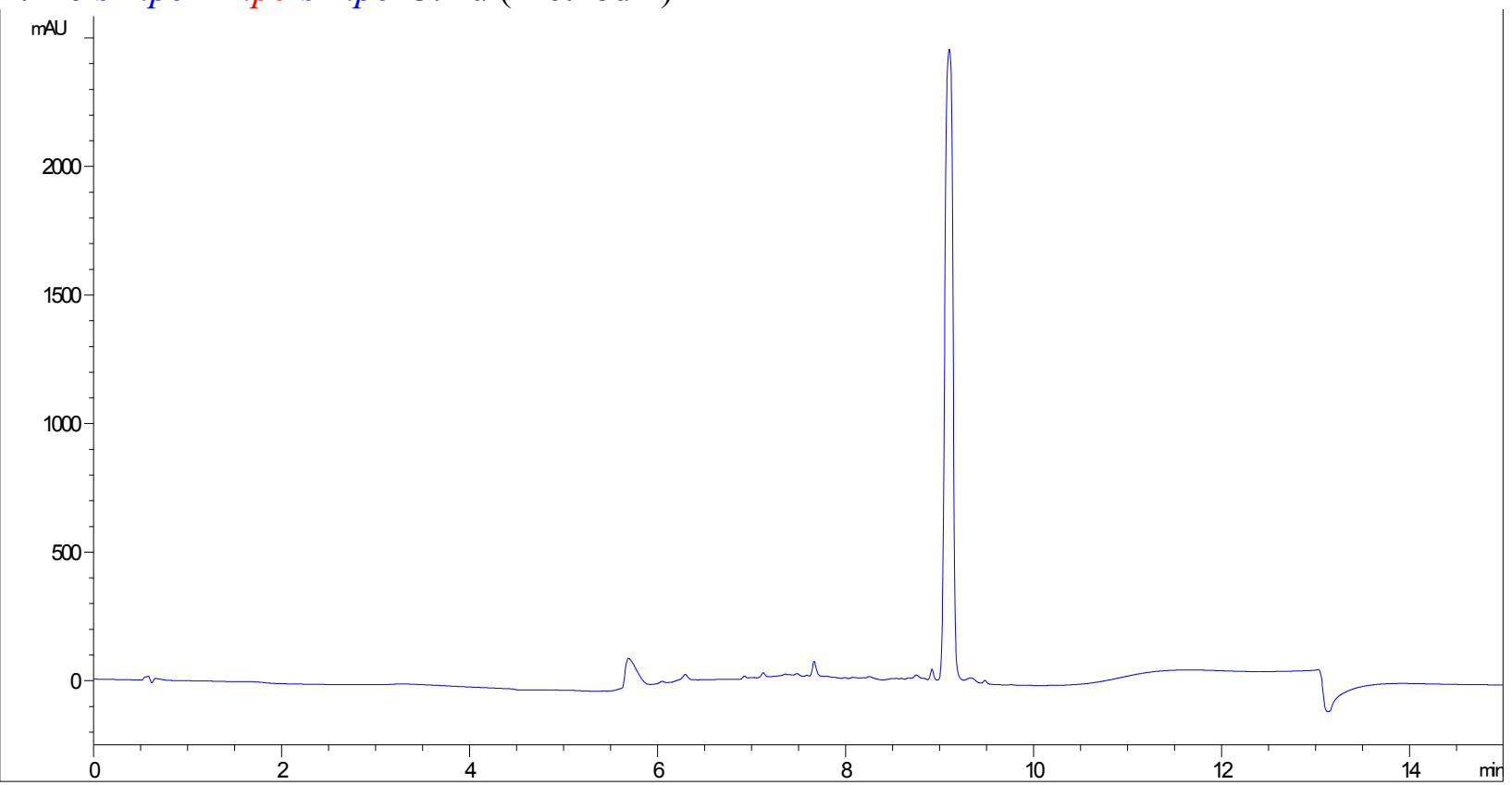


3: Ac-r1npe-s1npe-r1npe-s1npe- $\mathrm{O} t \mathrm{Bu}$ (Gradient elution: $50 \% \mathrm{~A} / 50 \% \mathrm{~B}$ for $3 \mathrm{~min}, 50 \% \mathrm{~A} / 50 \%$ to $100 \%$ B over $15 \mathrm{~min}, 100 \%$ B for $10 \mathrm{~min}$; flow rate of $1.0 \mathrm{~mL} / \mathrm{min}$.)

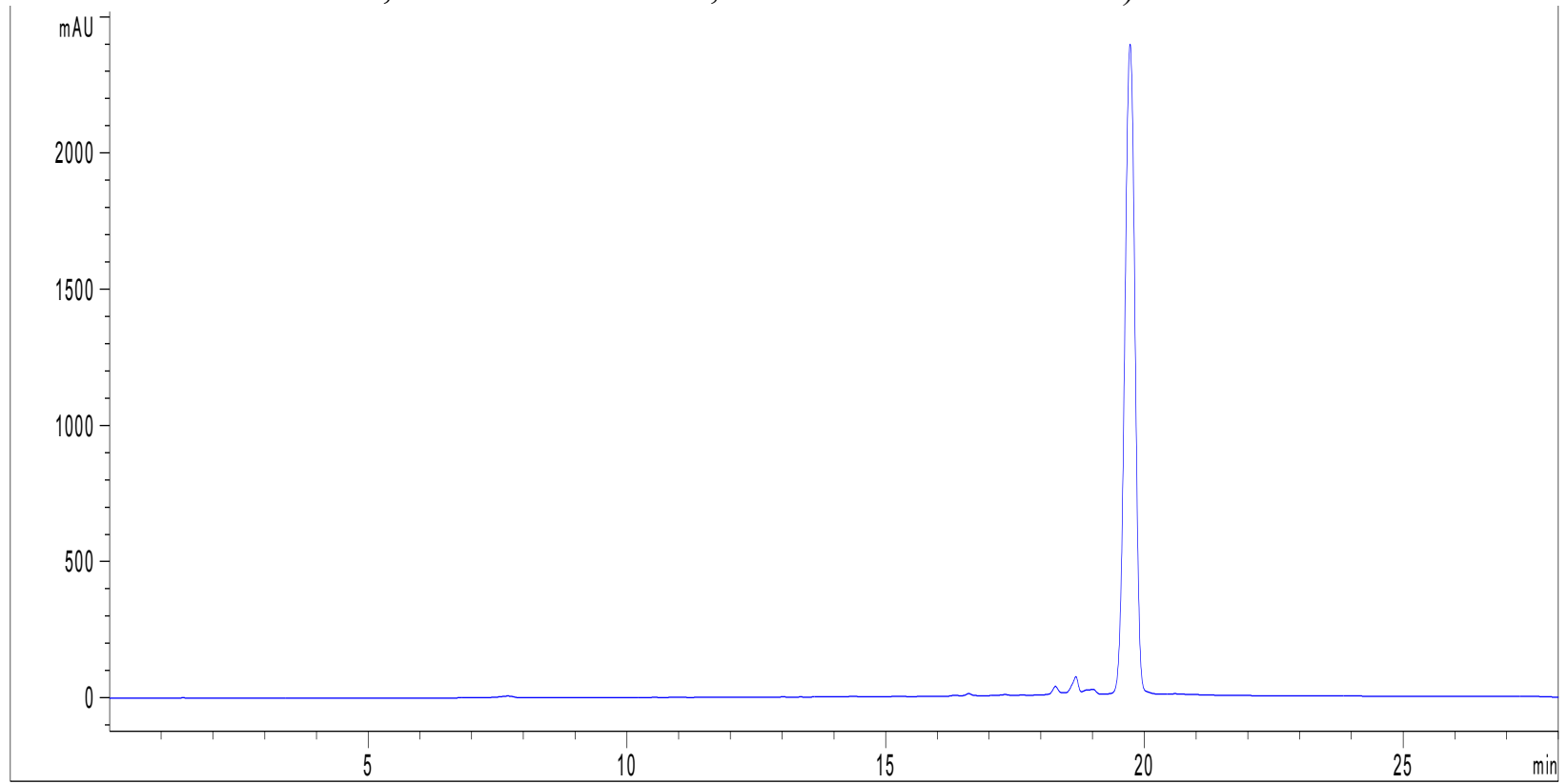

4: Ac-s1npe-r1npe-s1npe-r1npe-s1npe-O $t \mathrm{Bu}$ (Gradient elution: $20 \% \mathrm{~A} / 80 \% \mathrm{~B}$ for $3 \mathrm{~min}, 20 \%$ $\mathrm{A} / 80 \%$ to $100 \% \mathrm{~B}$ over $7 \mathrm{~min}, 100 \% \mathrm{~B}$ for $8 \mathrm{~min}$; flow rate of $1.0 \mathrm{~mL} / \mathrm{min}$.)

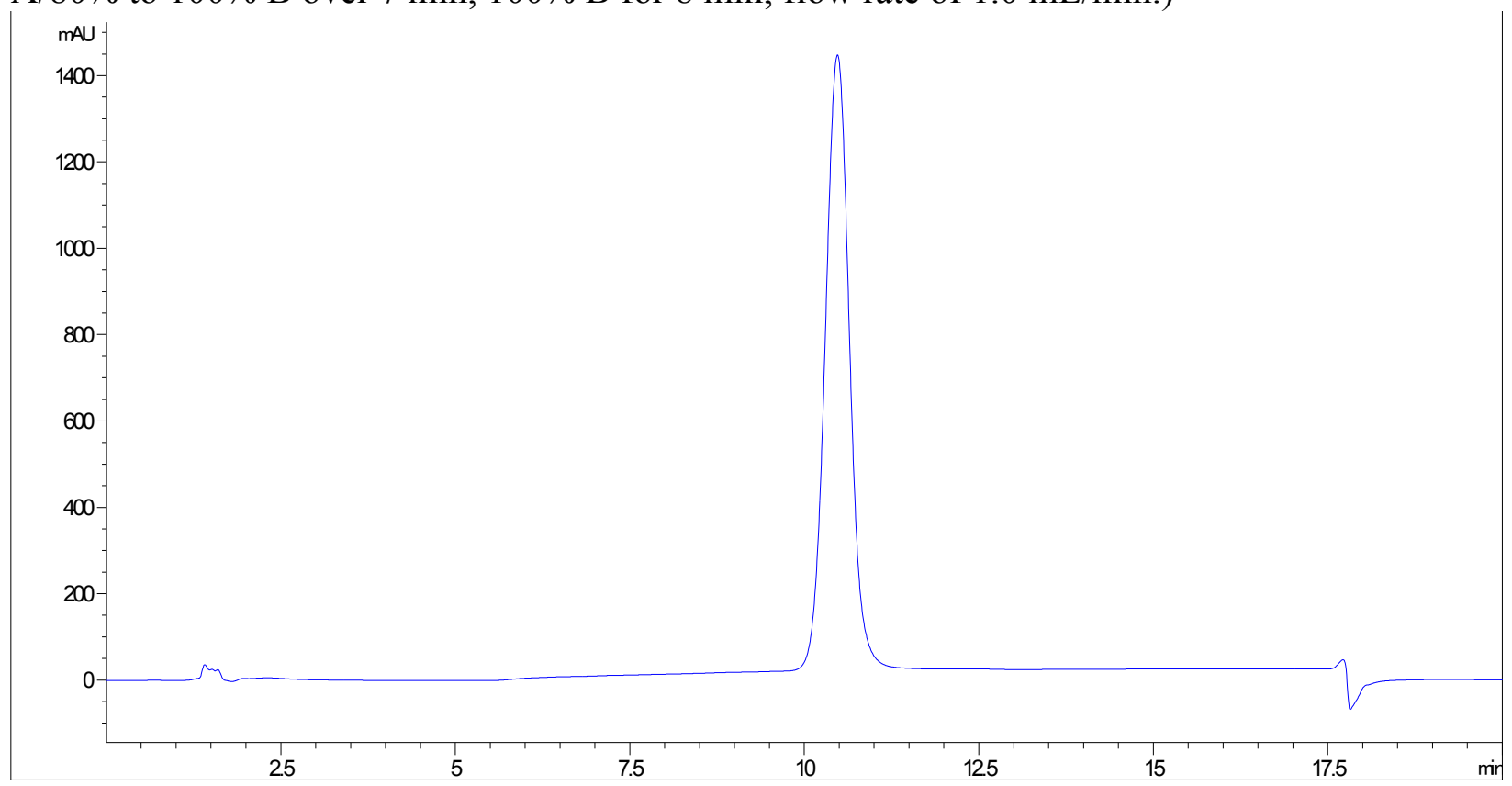


5: Ac-s1npe-r1npe-s1npe-NMe $\mathrm{NM}_{2}$ (Isocratic elution at 100\% B for $45 \mathrm{~min}$; flow rate of 1.0 $\mathrm{mL} / \mathrm{min}$.)

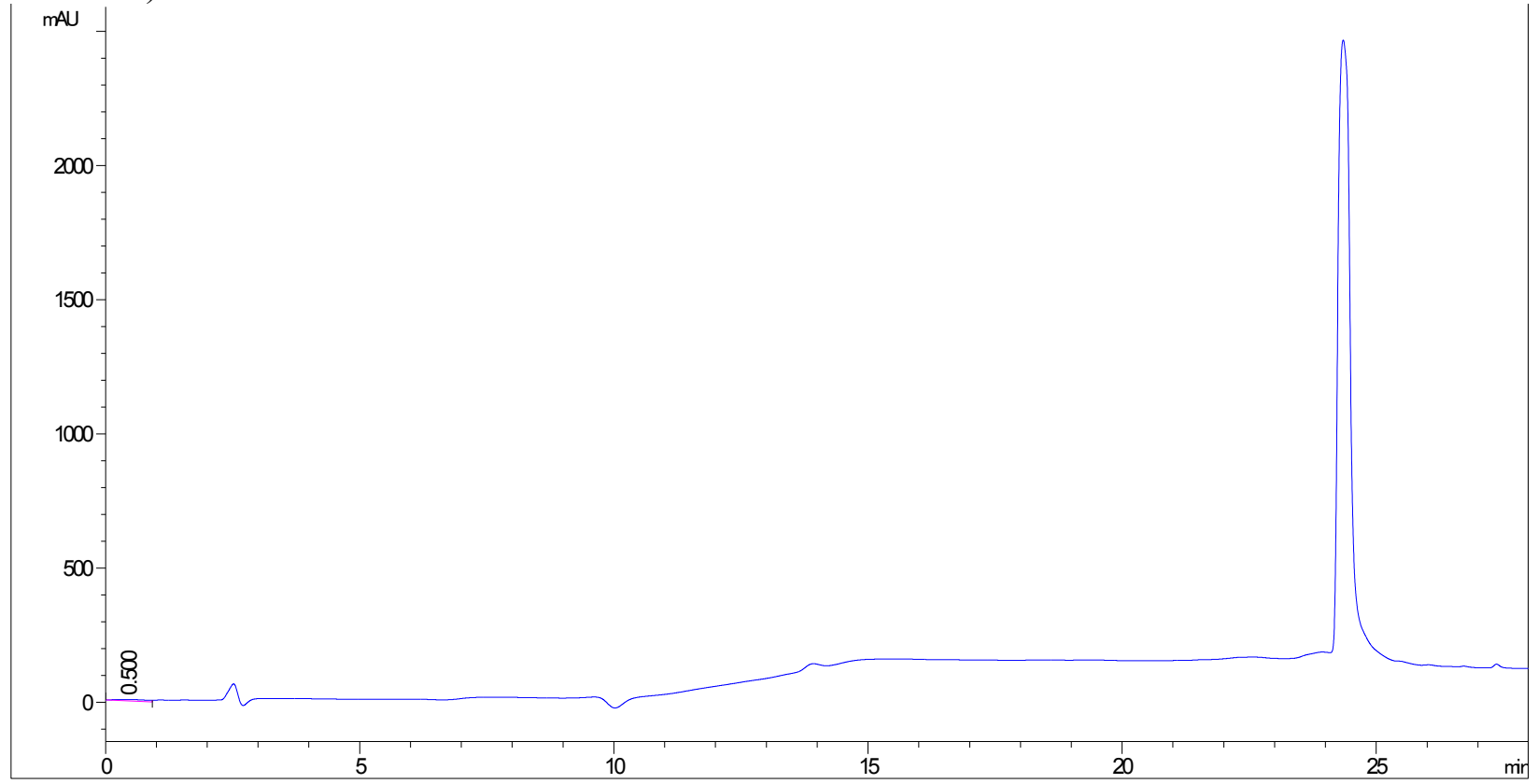

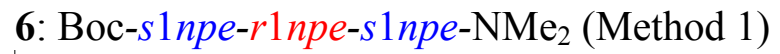

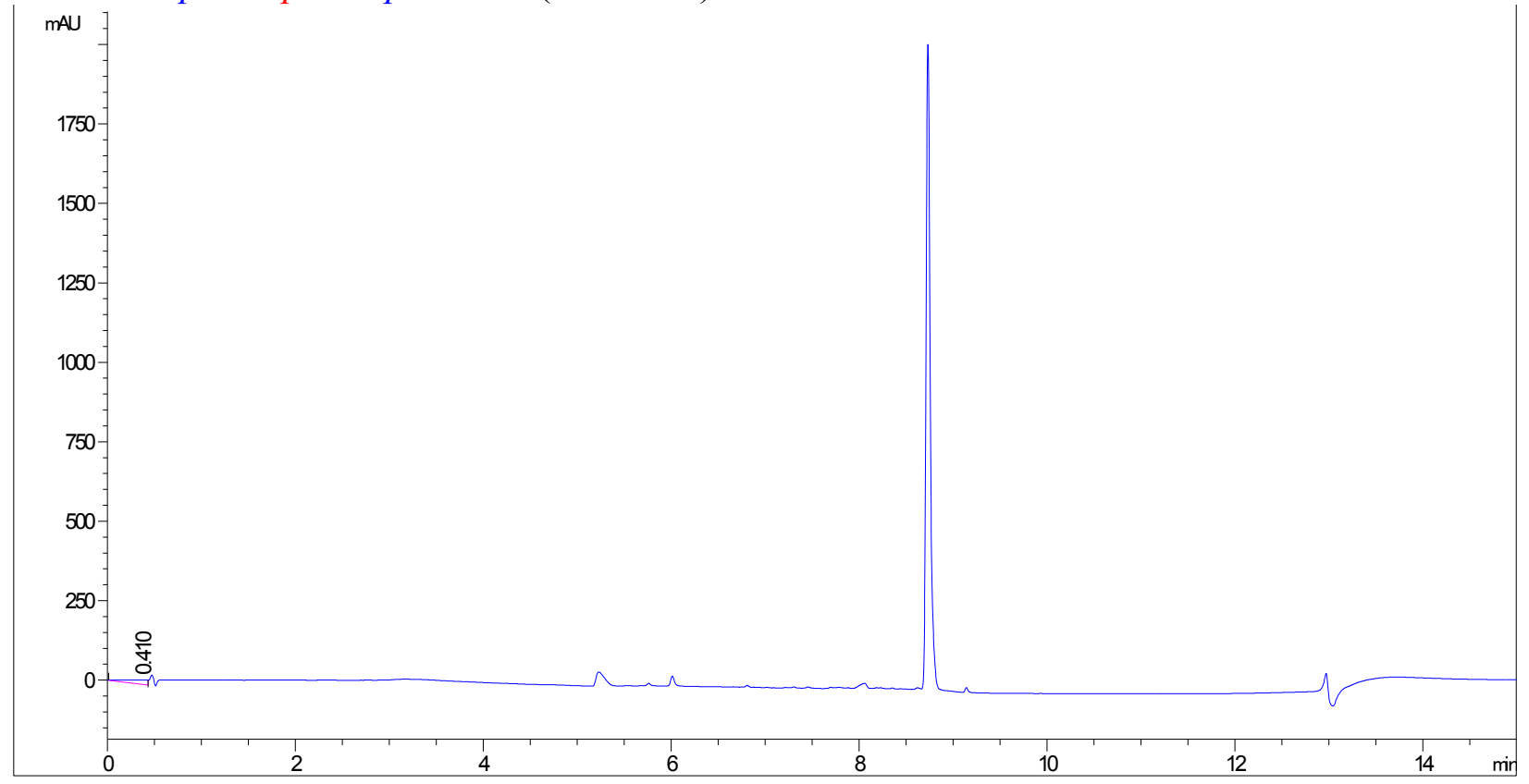


7: Ac-r1npe-s1npe-H (Method 1)

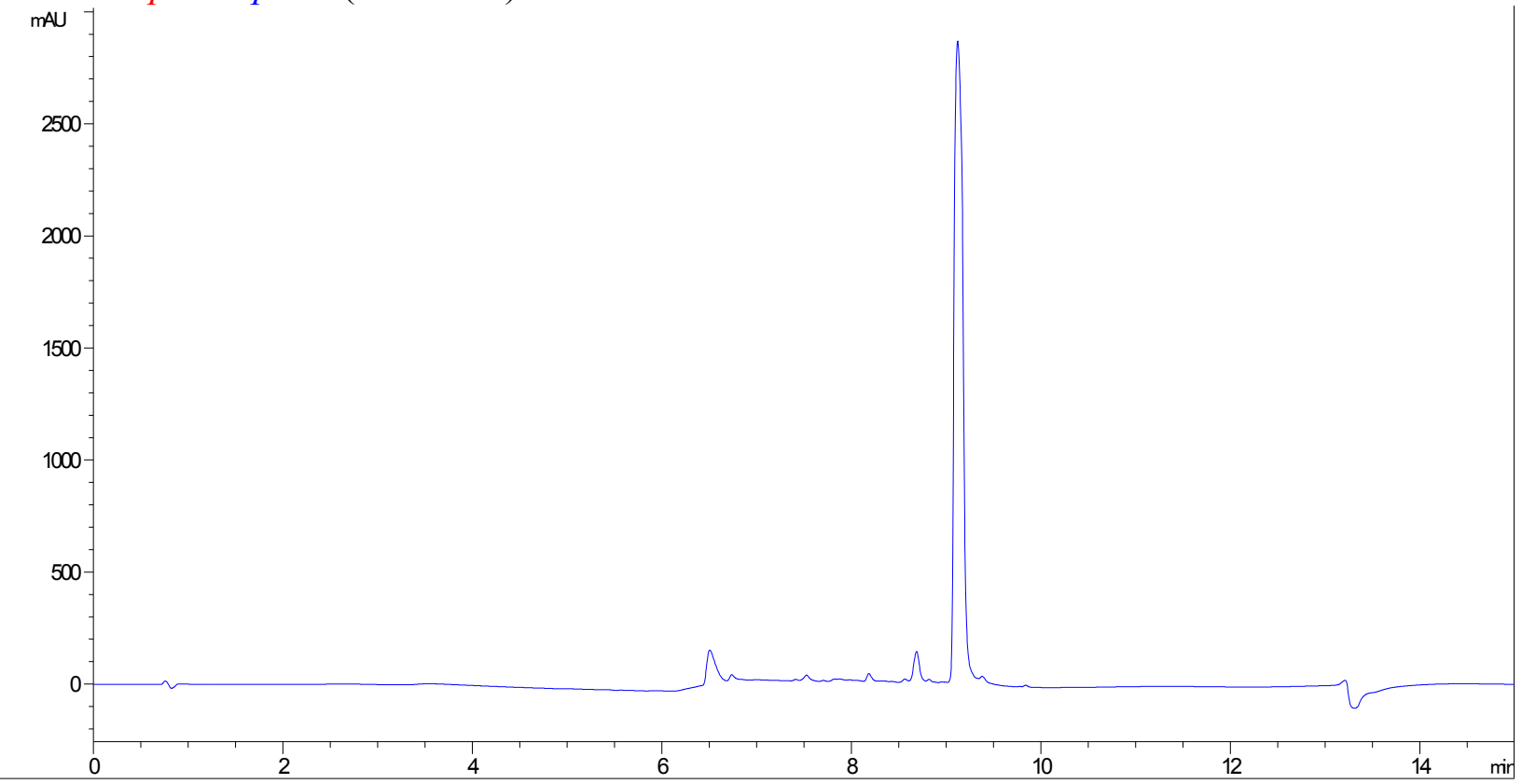

\section{8: Ac-s1npe-r1npe-s1npe-H (Method 2)}

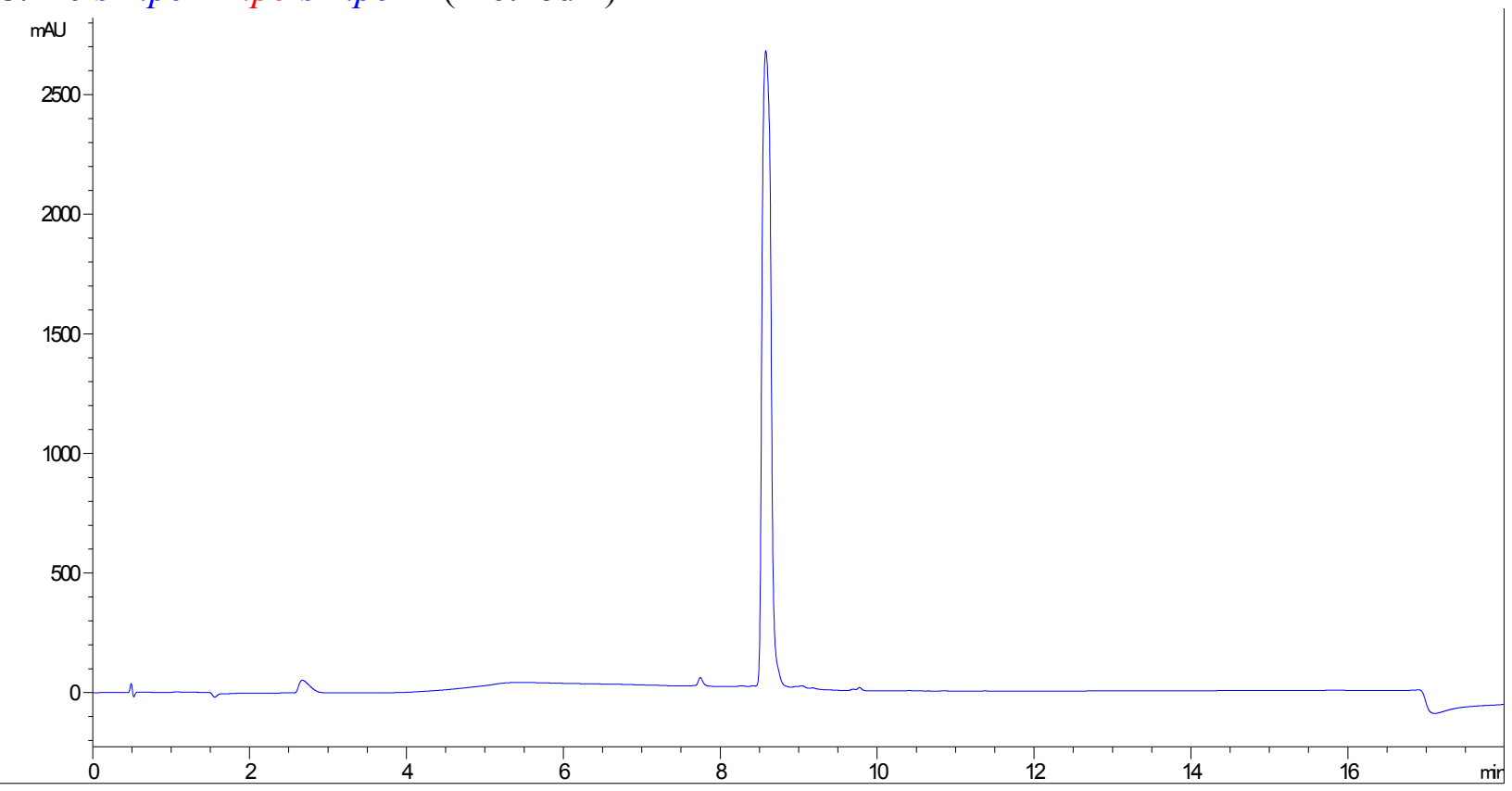

S- 8 
9: Ac-r1npe-s1npe-r1npe-s1npe-H (Method 1)

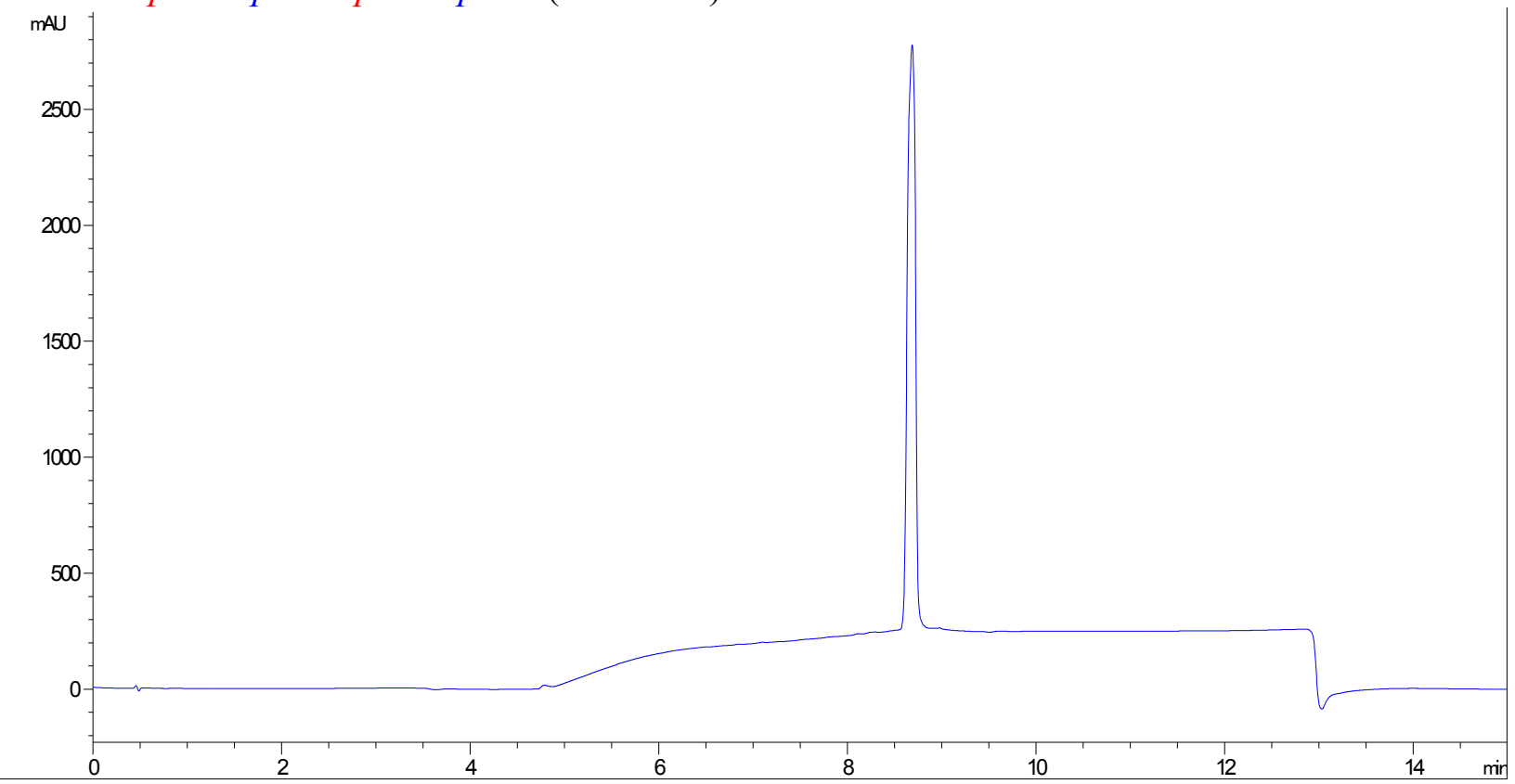

10: Ac-s1npe-r1npe-s1npe-r1npe-s1npe-H (Method 1)

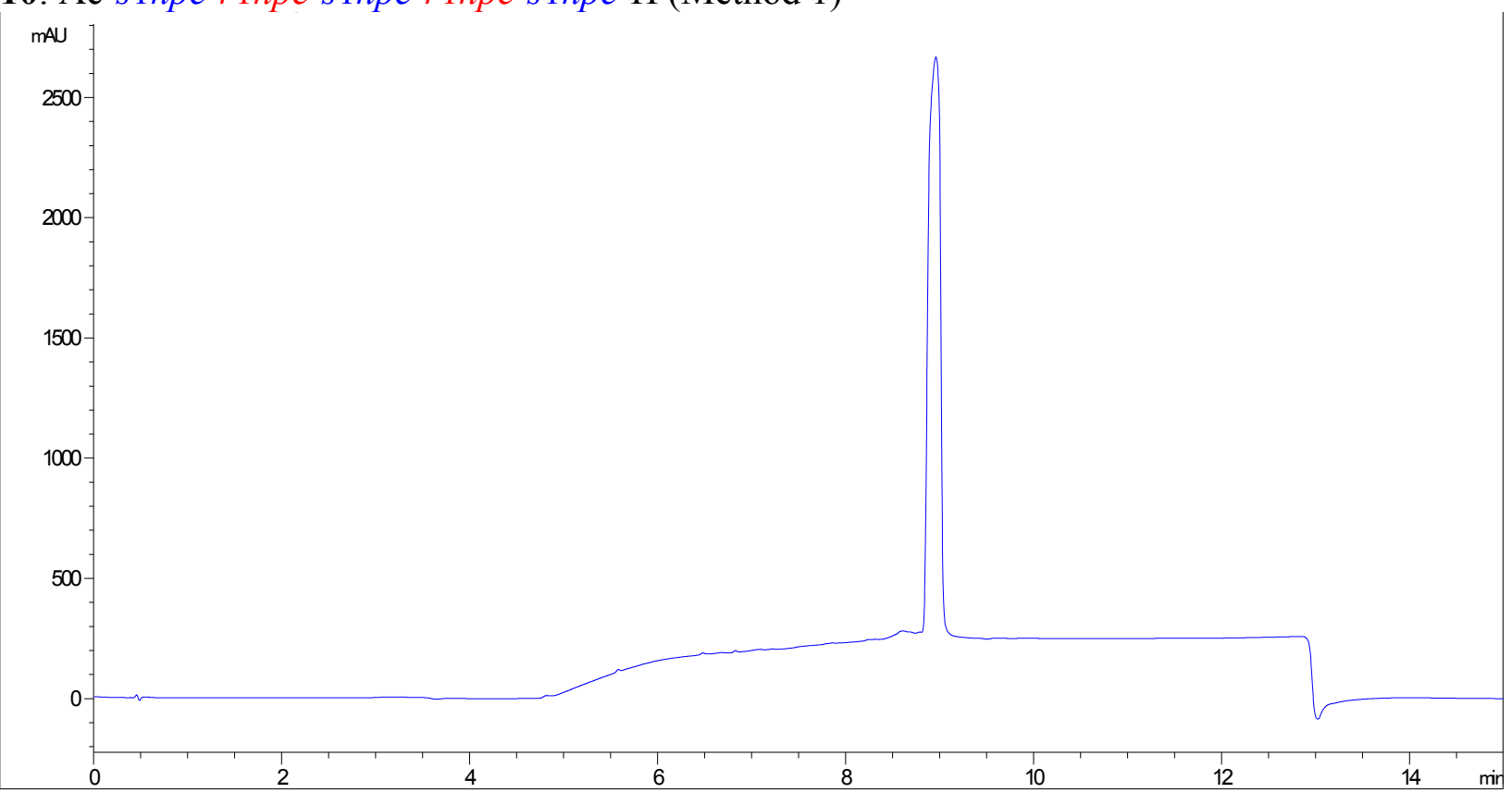


11: Ac-spe-rpe-spe-H (Method 1)

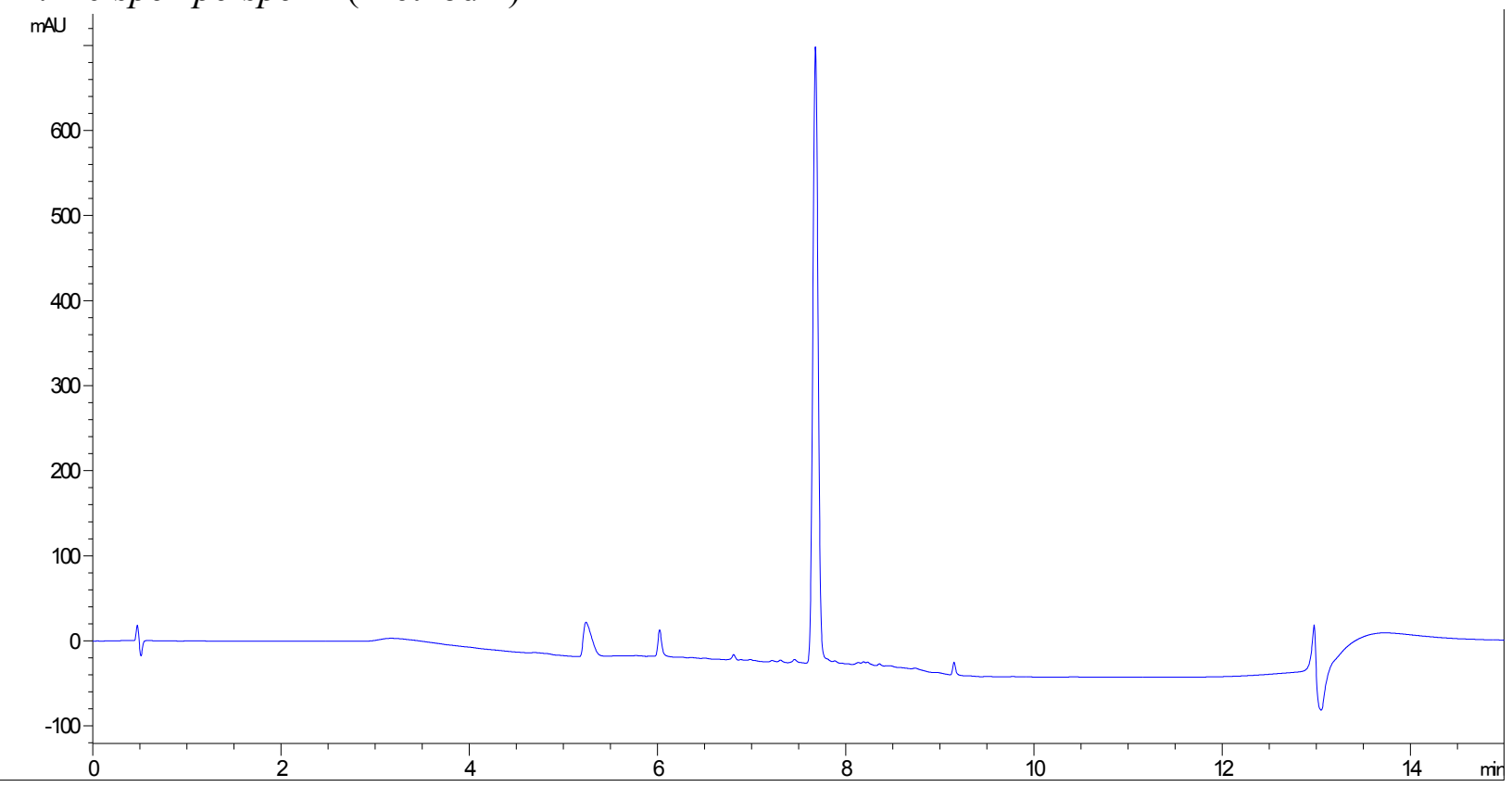

12: Ac-r1npe-r1npe-s1npe-s1npe-H (Method 2)

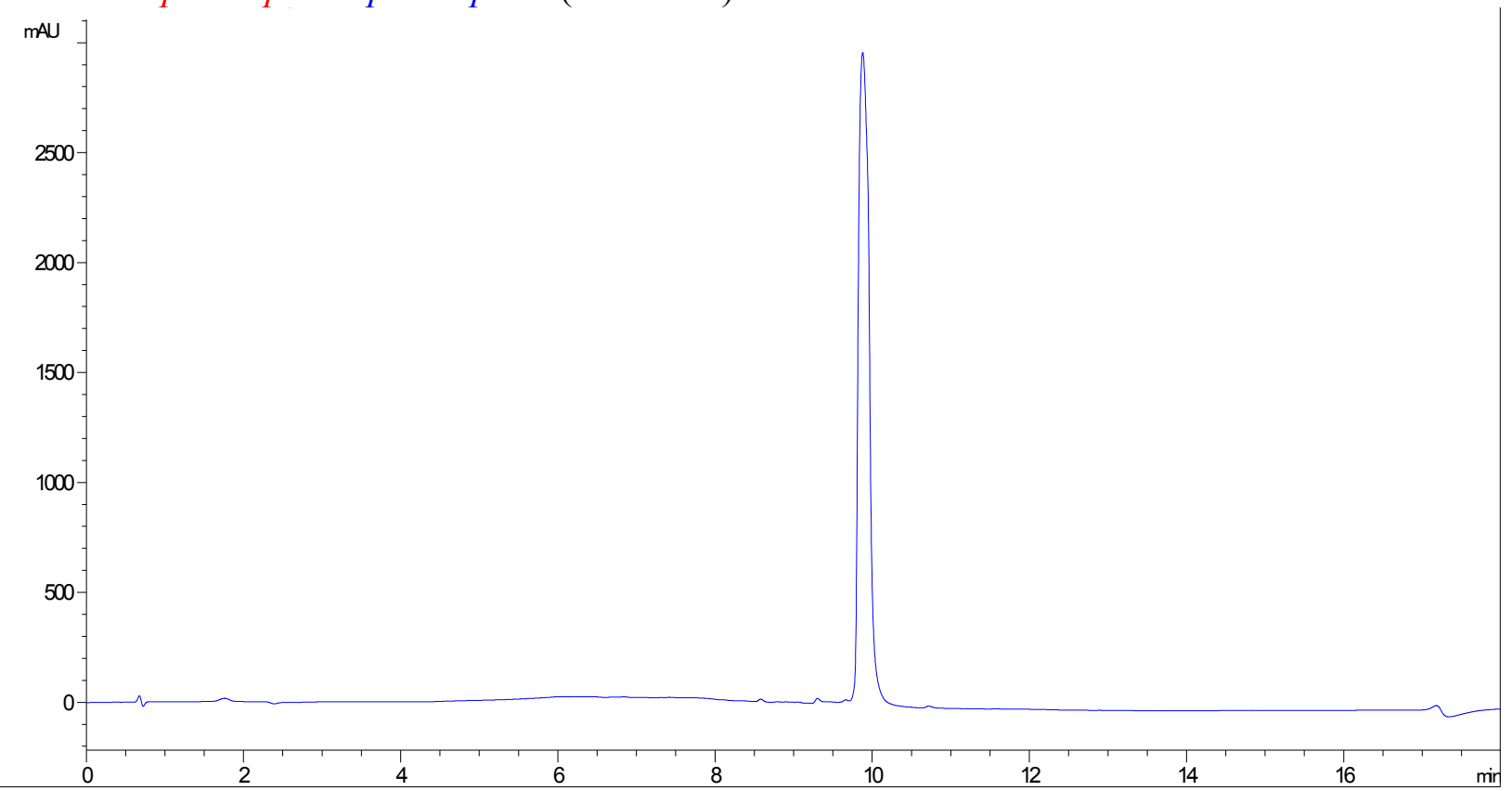


13: Boc-r1npe-r1npe-s1npe-s1npe-H (Method 1)

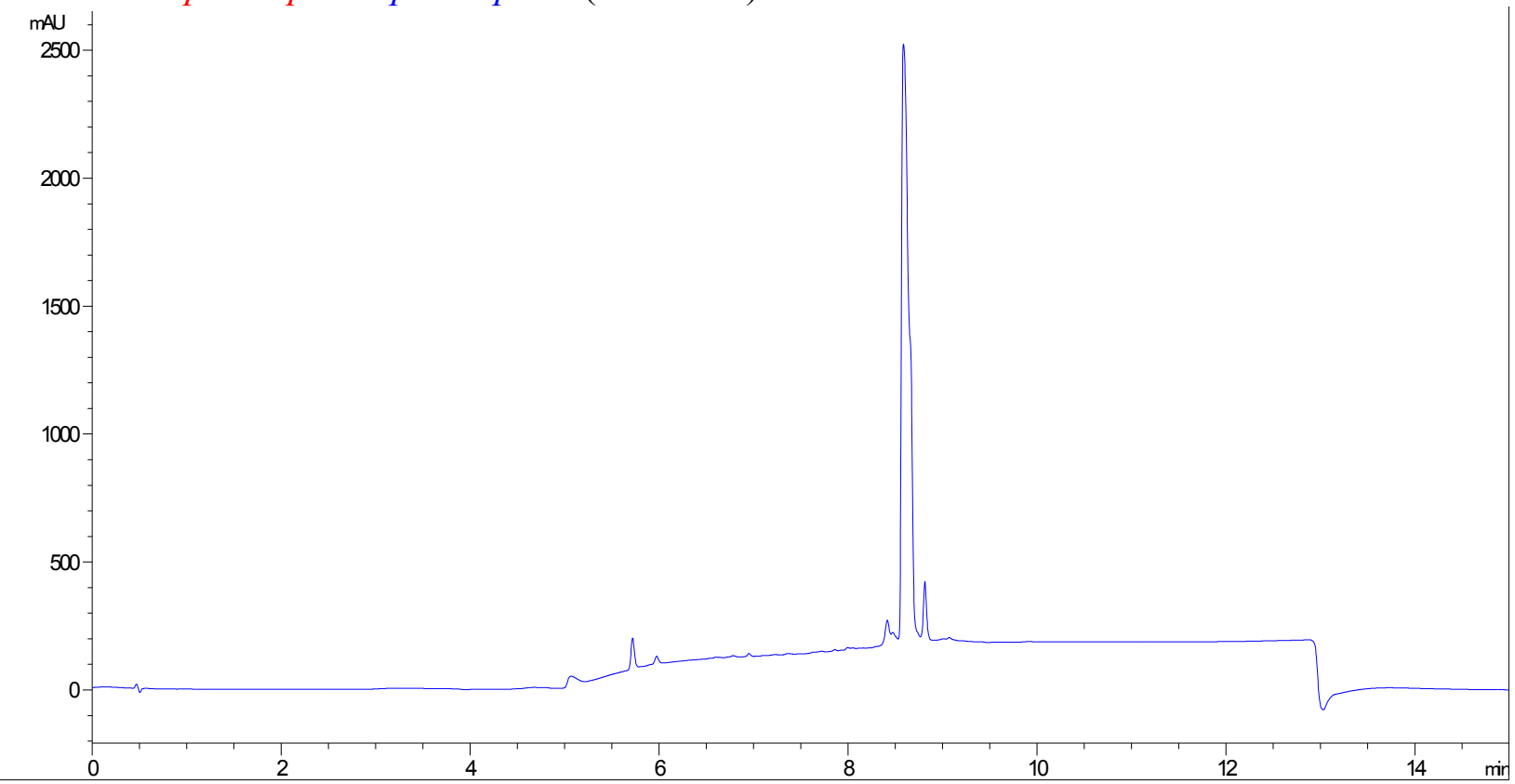

14: Boc-s1npe-r1npe-s1npe-r1npe-s1npe-H (Method 1)

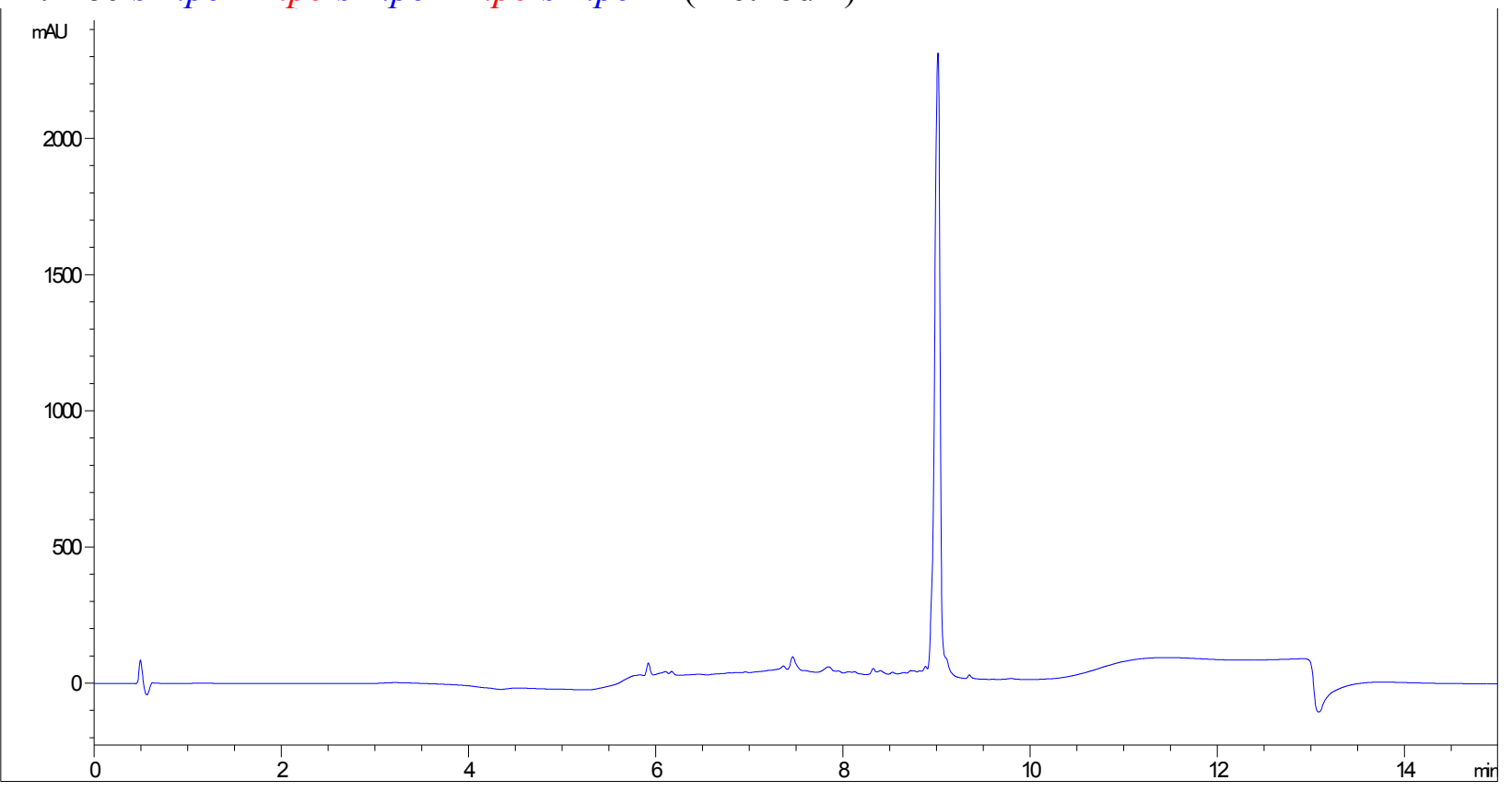


NMR spectra and analysis. COSY experiments (Z-gradient selection) were performed at $24{ }^{\circ} \mathrm{C}$ using the following parameter values: spectral width $=20.69 \mathrm{ppm}$; number of transients $(\mathrm{nt})=2$; number of increments $(\mathrm{ni})=256$; relaxation delay $=5 \mathrm{~s}$. The number of points (np) was 2048. HSQC experiments were performed at $24{ }^{\circ} \mathrm{C}$ using the following parameter values: mix time $=300 \mathrm{~ms}$; spectral width $=8278 \mathrm{~Hz}$; number of transients $(\mathrm{nt})=64$; number of increments (ni) $=256$; relaxation delay $=2.5 \mathrm{~s}$. The number of points (np) was 2048 .

Edited ge-HSQC experiments (multiplicity editing; phase-selection using echo/antiechoTPPI gradient selection) were performed at $24{ }^{\circ} \mathrm{C}$ using the following parameter values: spectral widths $=20.69$ and $165.64 \mathrm{ppm}$; number of transients $(\mathrm{nt})=32$; number of increments $(\mathrm{ni})=$ 384; relaxation delay $=5 \mathrm{~s}$. The number of points (np) was 1024 . Amide cis/trans ratios were determined by averaging the integrations of at least two sets of rotamer-related peaks for each compound; these peaks were identified using previously established criteria ${ }^{4}$ and verified using the COSY and NOESY spectra.

HMBC experiments (low-pass $J$-filer; gradient pulses for selection) were performed at $24{ }^{\circ} \mathrm{C}$ using the following parameter values: spectral widths $=20.69$ and $165.64 \mathrm{ppm}$; number of transients $(\mathrm{nt})=32$; number of increments $(\mathrm{ni})=384$; relaxation delay $=5 \mathrm{~s}$.

NOESY experiments (phase sensitive using TPPI) were performed at $24^{\circ} \mathrm{C}$ using the following parameter values: spectral width $=20.69$; number of transients $(n t)=32$; number of increments $(\mathrm{ni})=512$; mixing time $=300 \mathrm{~ms}$; relaxation delay $=5 \mathrm{~s}$. Peaks in the NOESY spectra of $\mathbf{2}$ and $\mathbf{8}$ were unambiguously assigned by analysis of the COSY, HSQC, and HMBC spectra. 


\section{1: ${ }^{1} \mathrm{H}$ NMR}

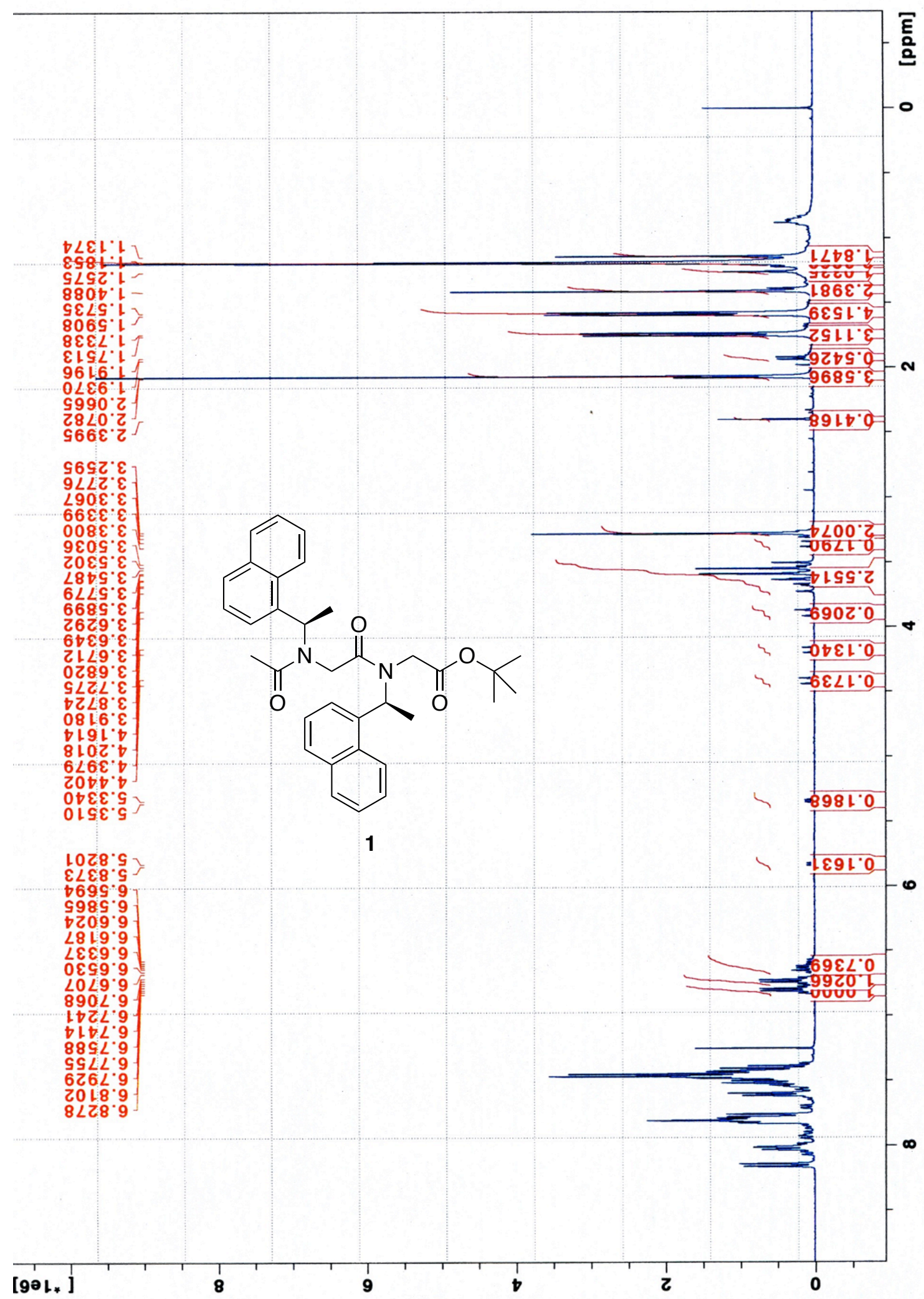

S- 13 


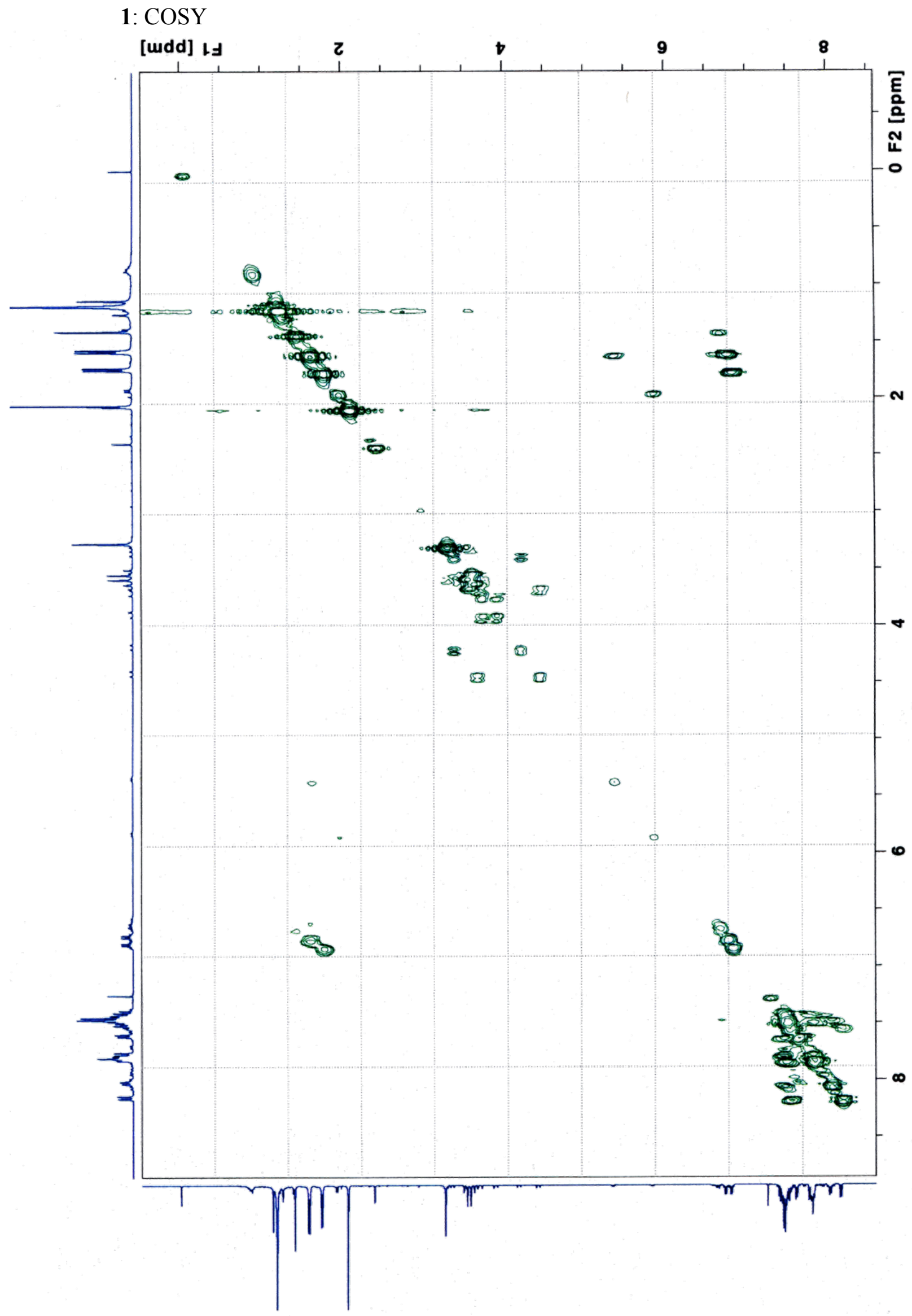

S- 14 


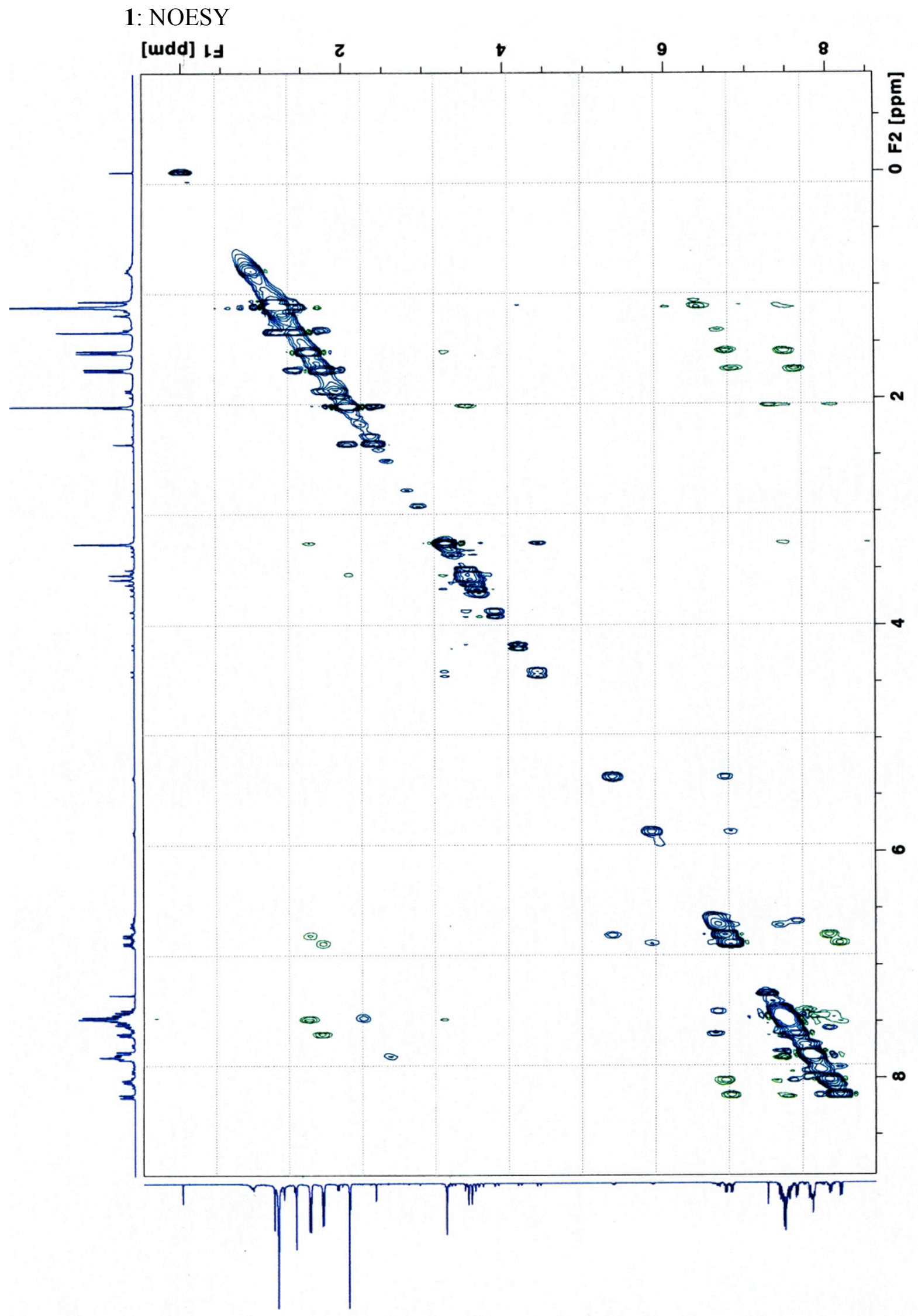

S- 15 


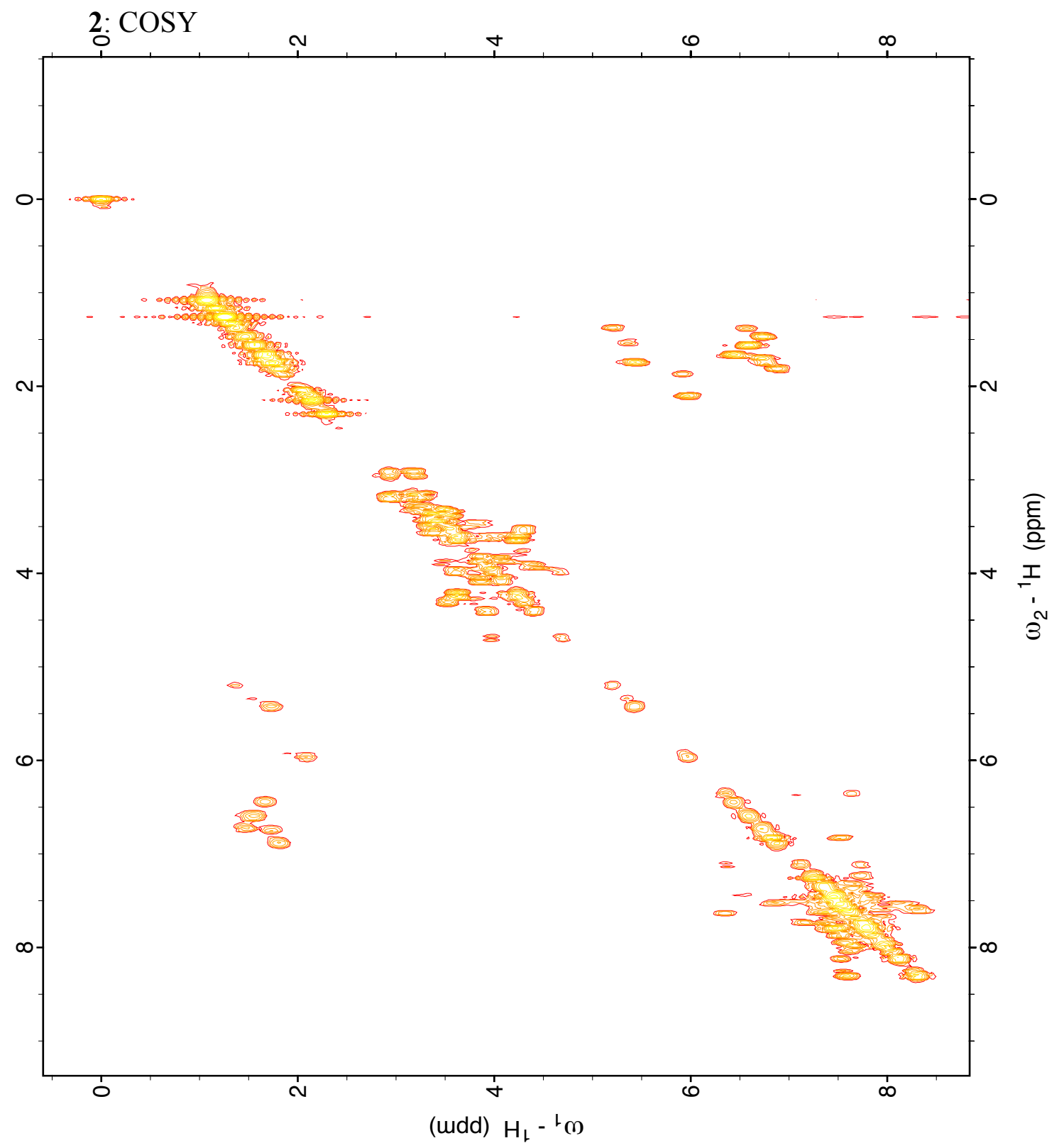

S- 16 


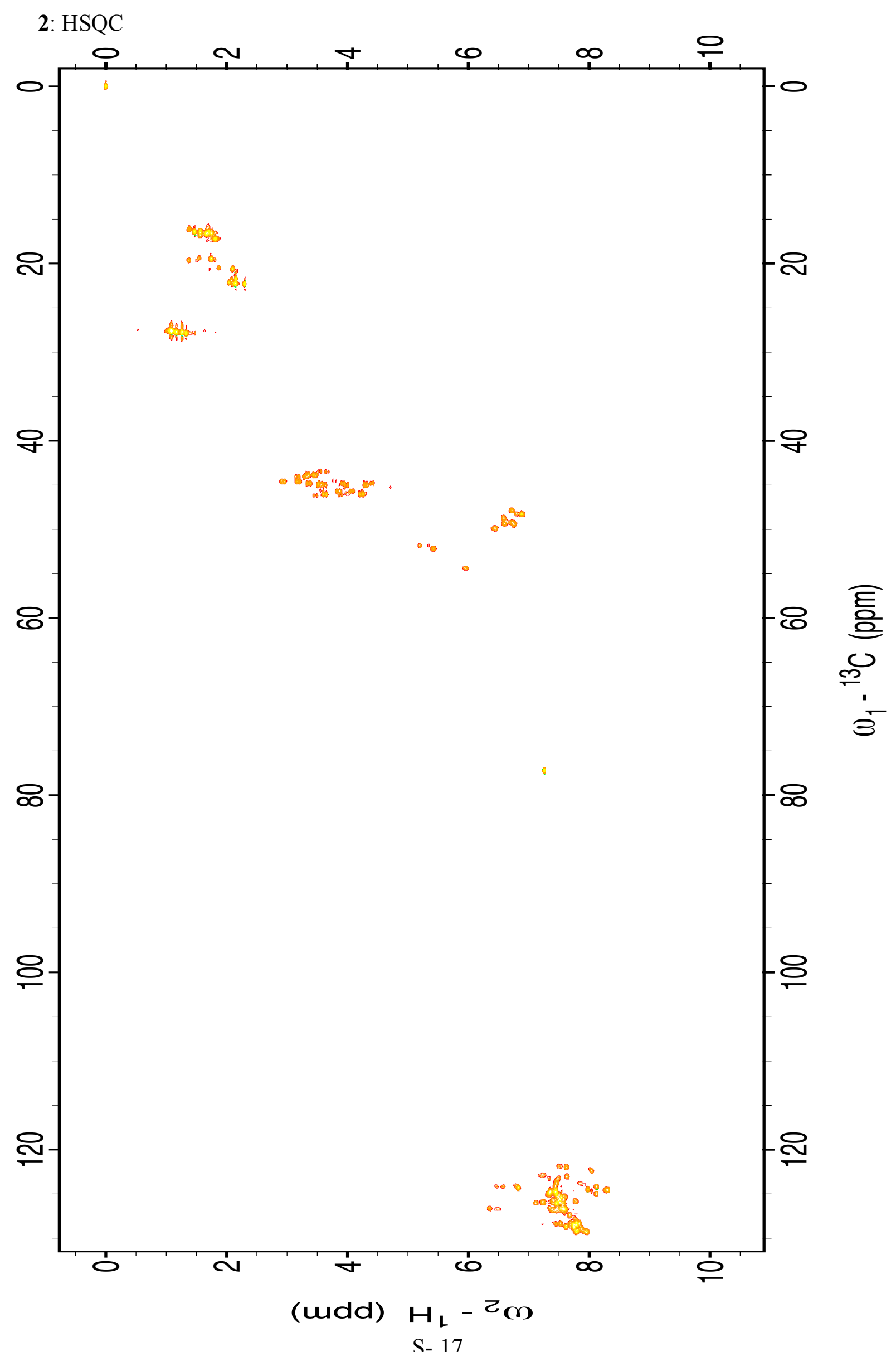




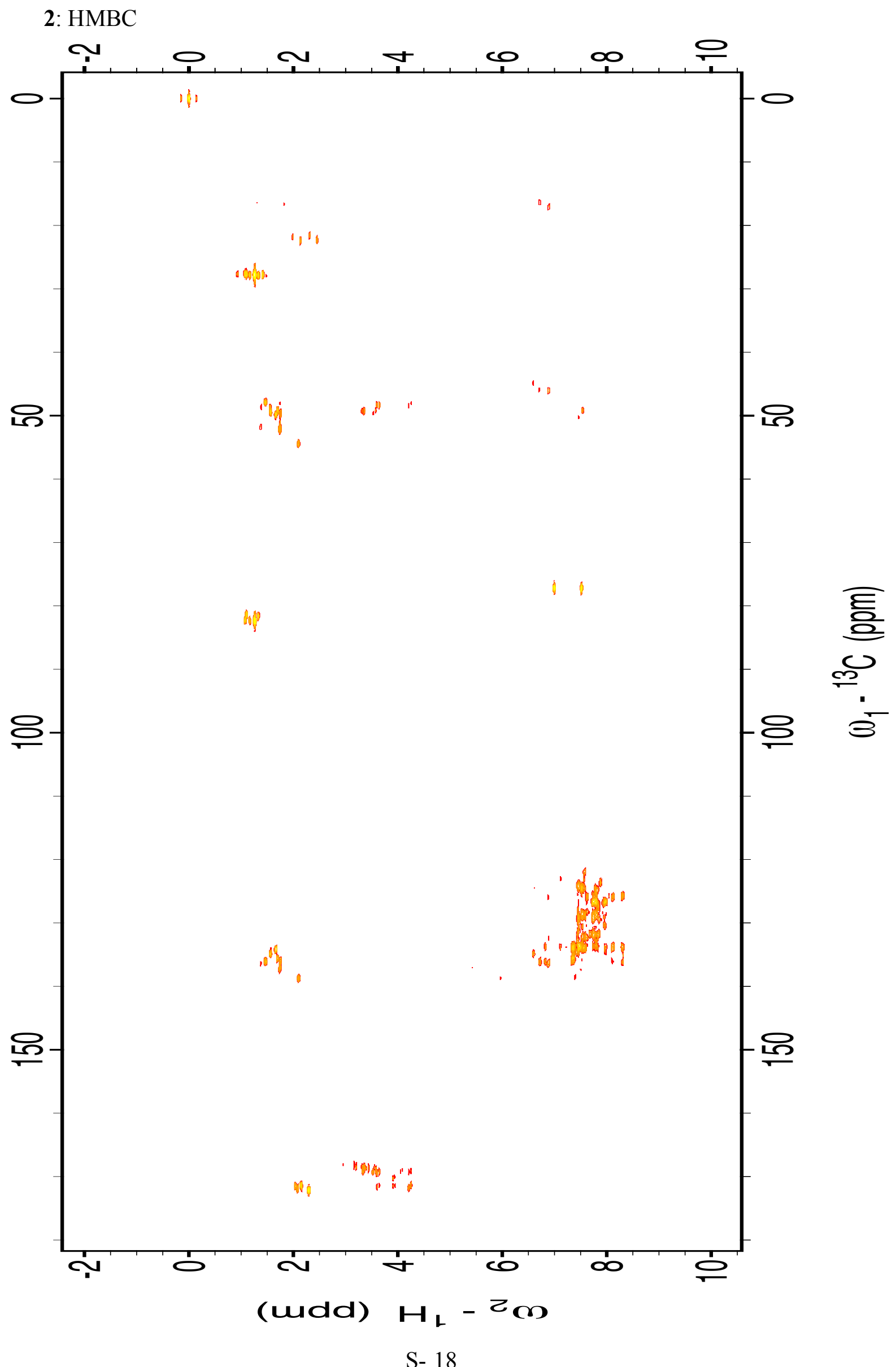




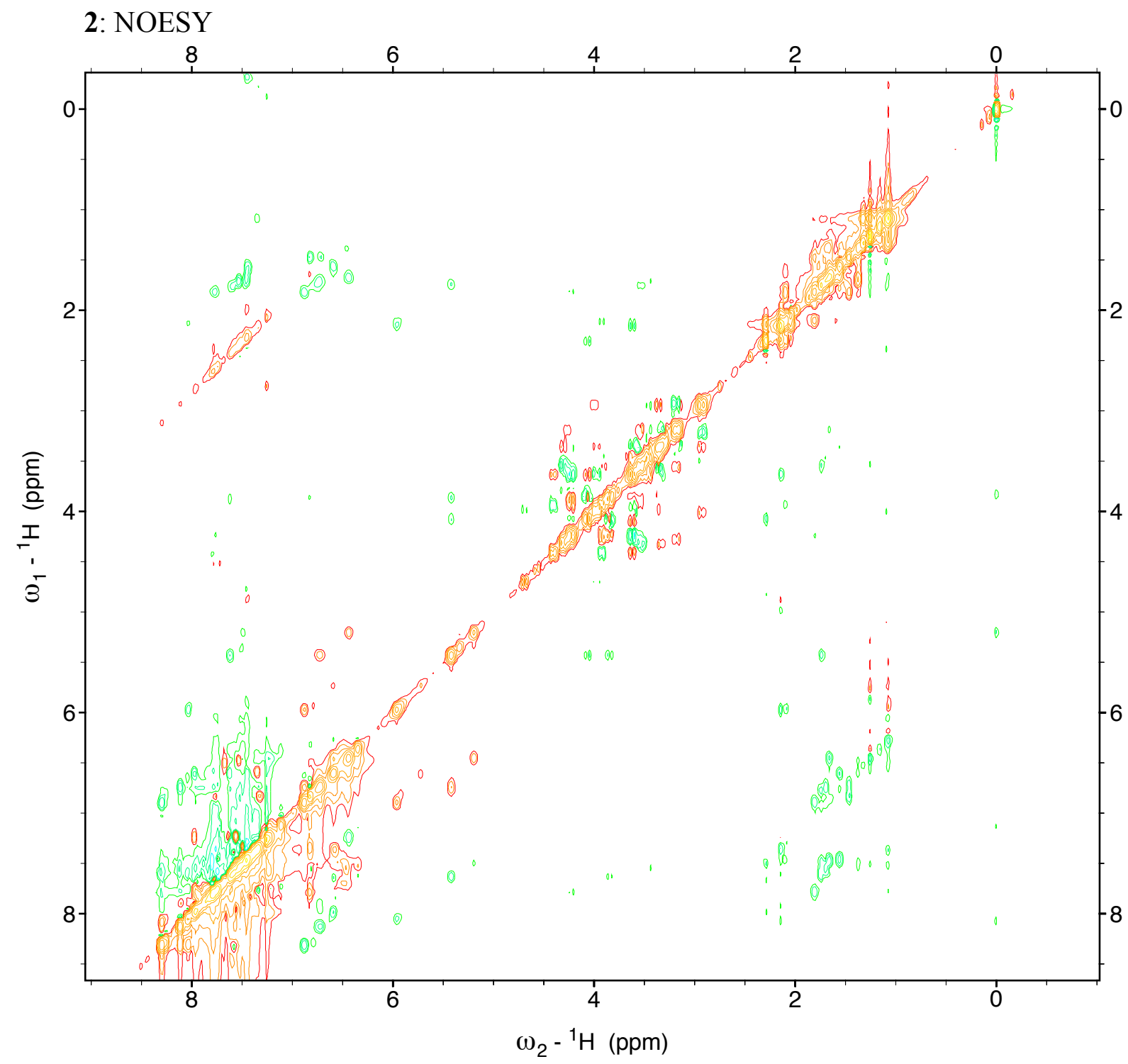

S- 19 


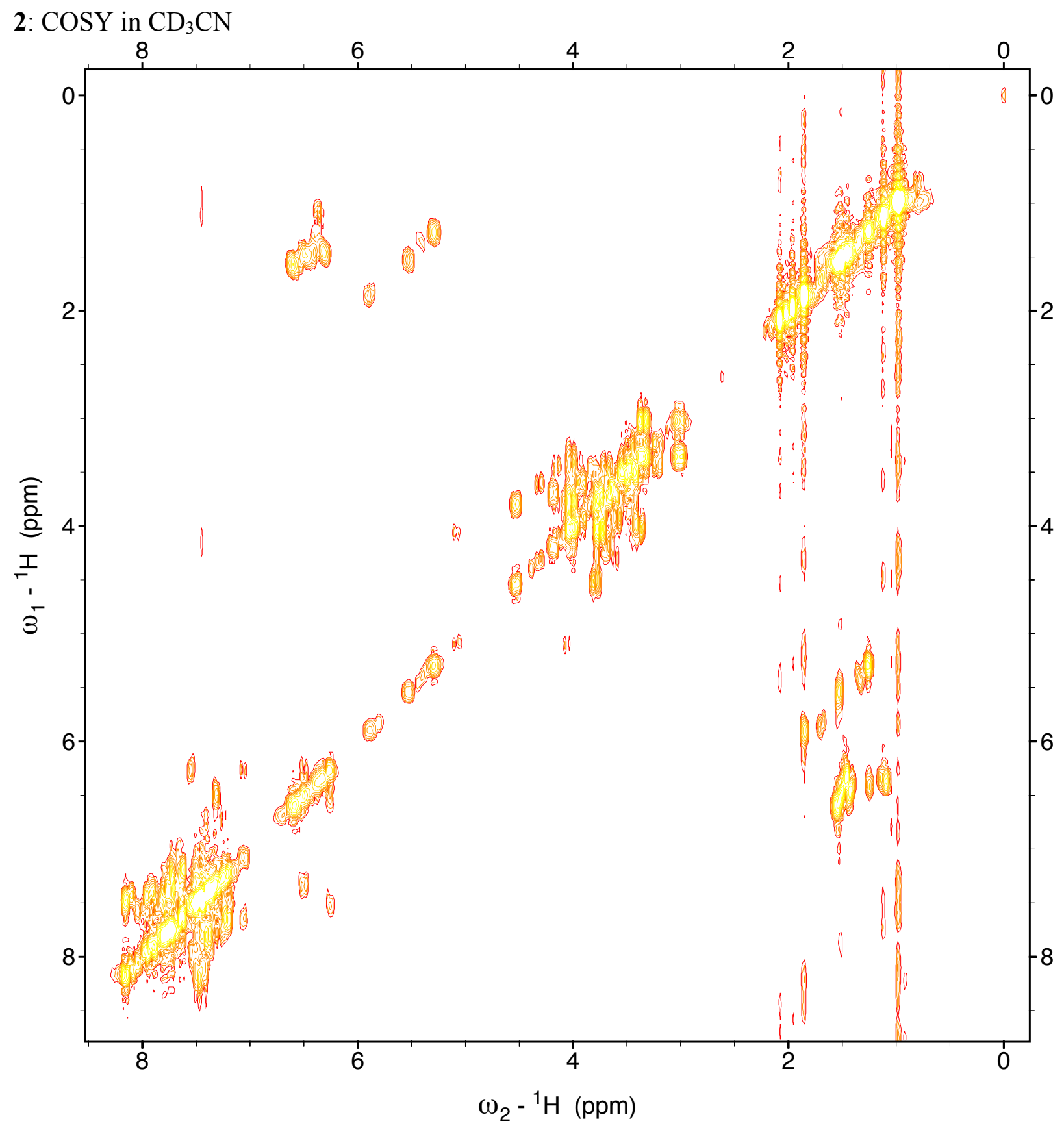


2: $\mathrm{HSQC}$ in $\mathrm{CD}_{3} \mathrm{CN}$

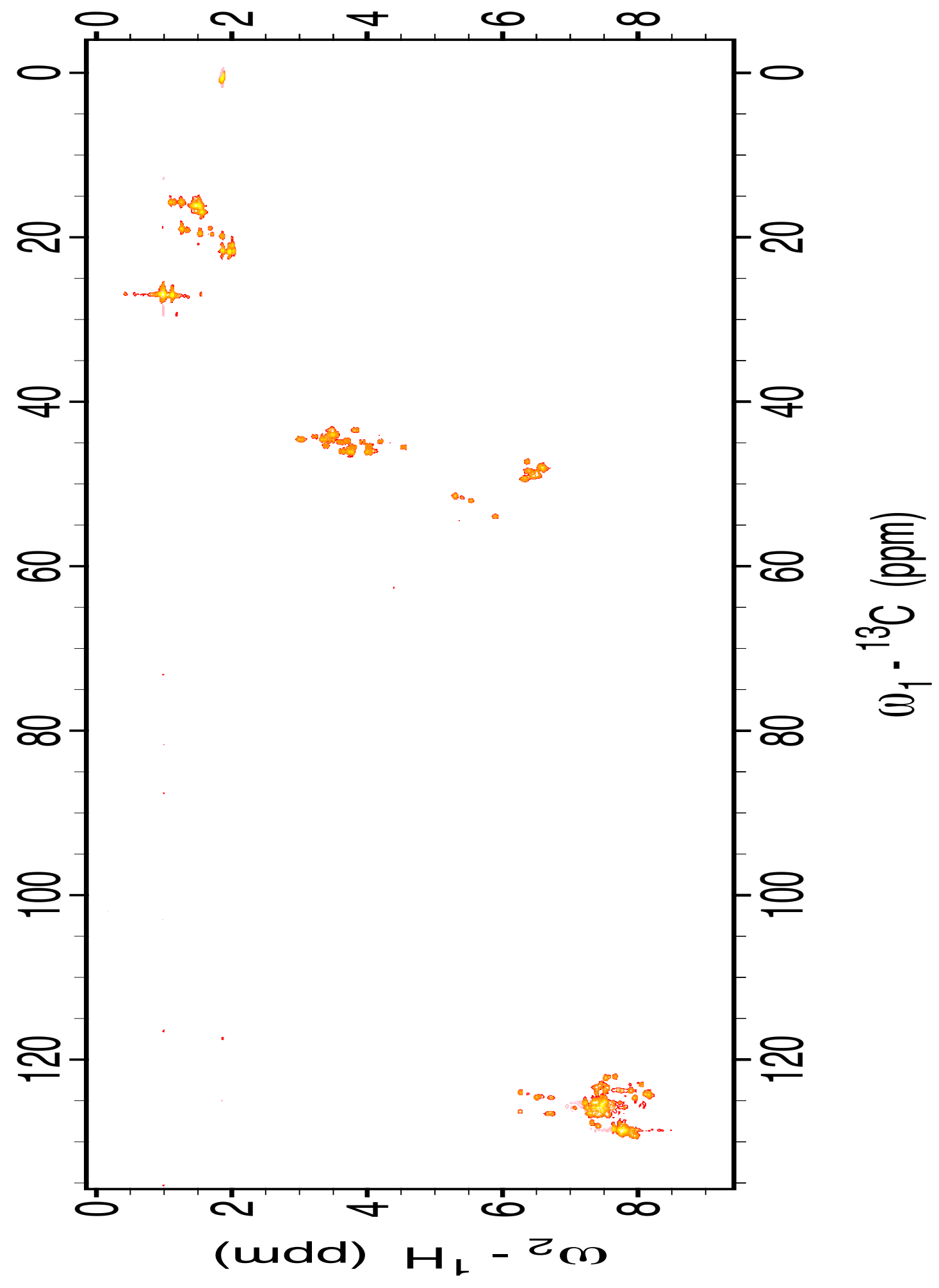


2: $\mathrm{HMBC}$ in $\mathrm{CD}_{3} \mathrm{CN}$

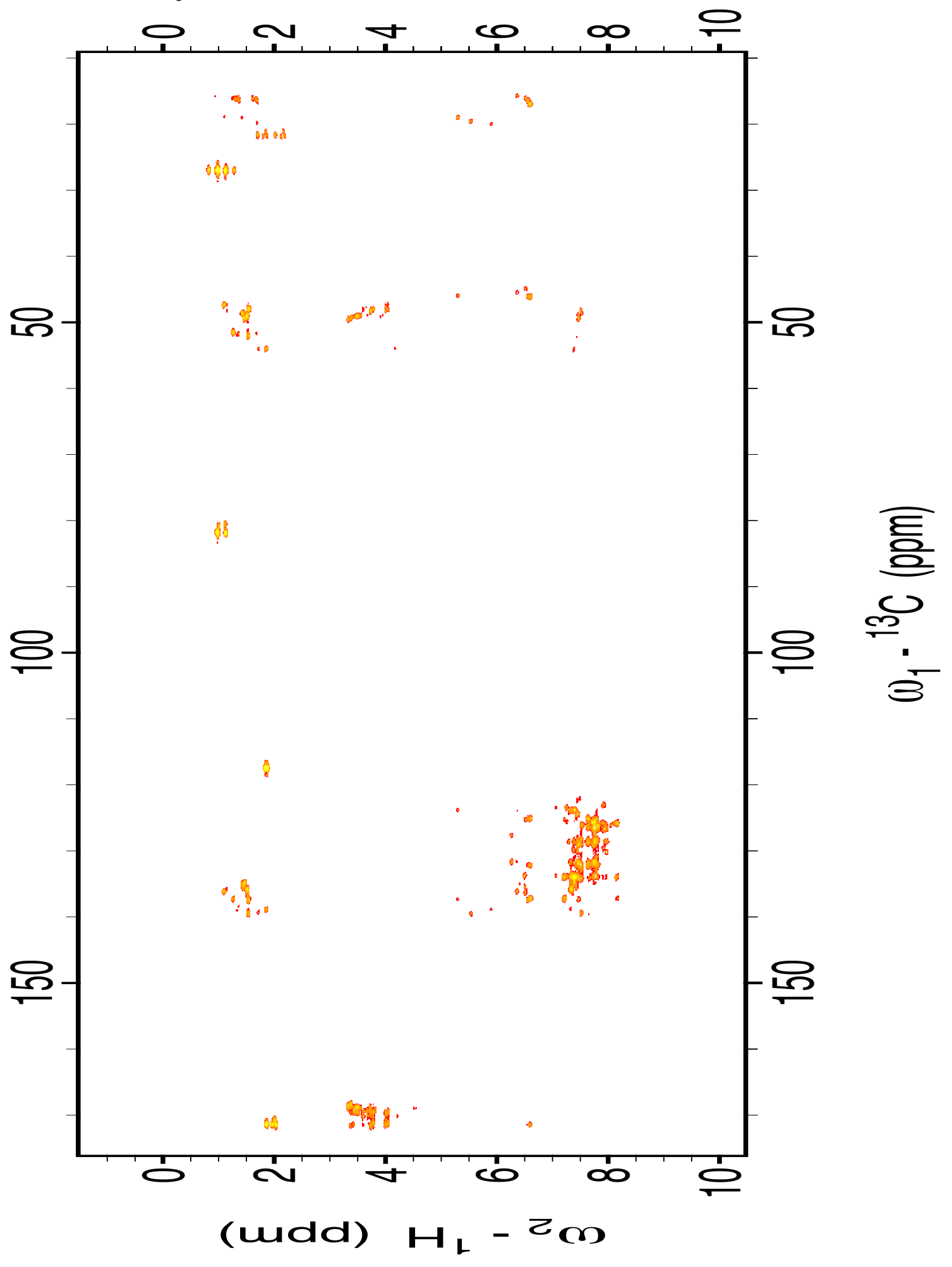




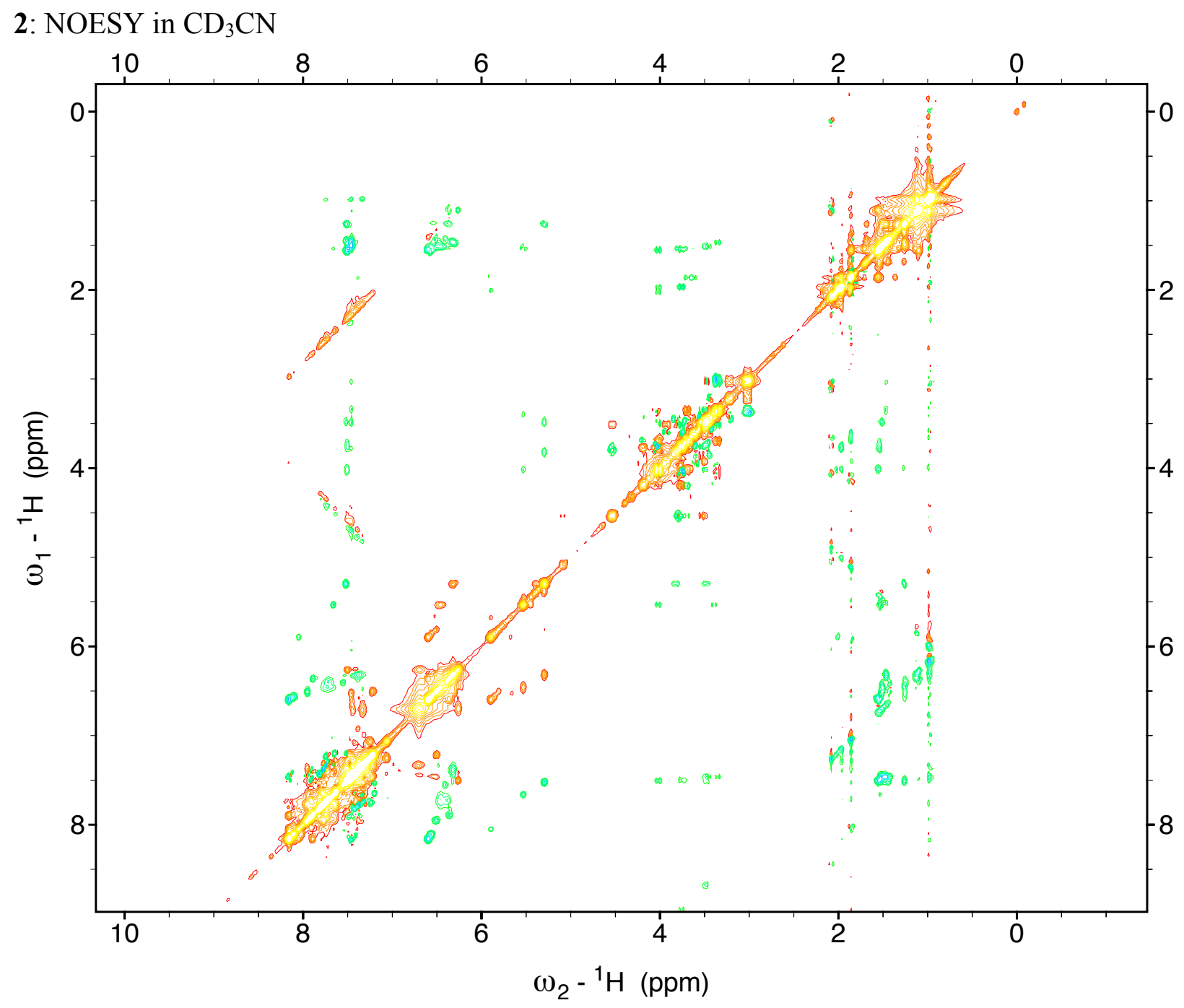

S- 23 


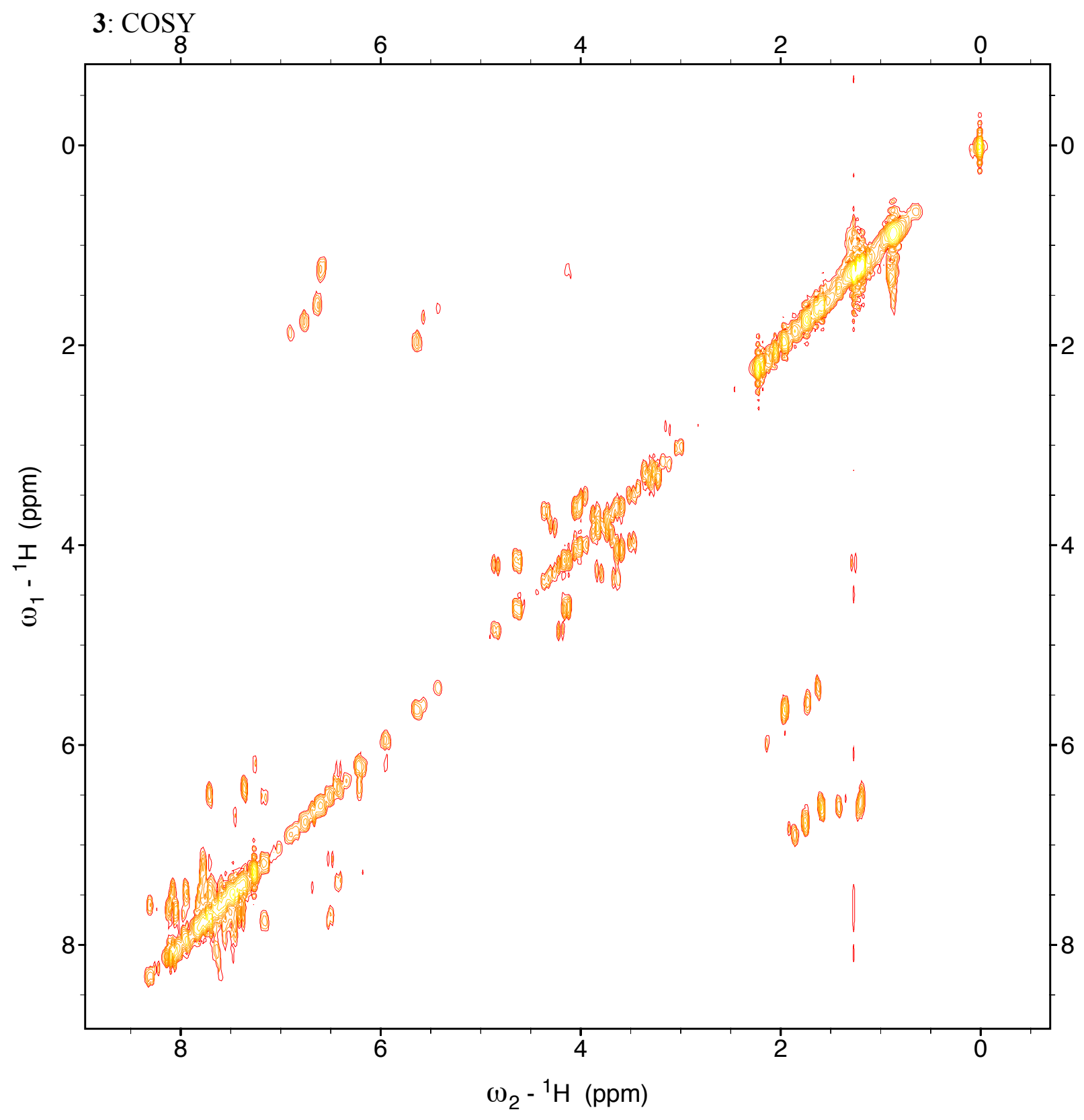

S- 24 


\section{3: NOESY}

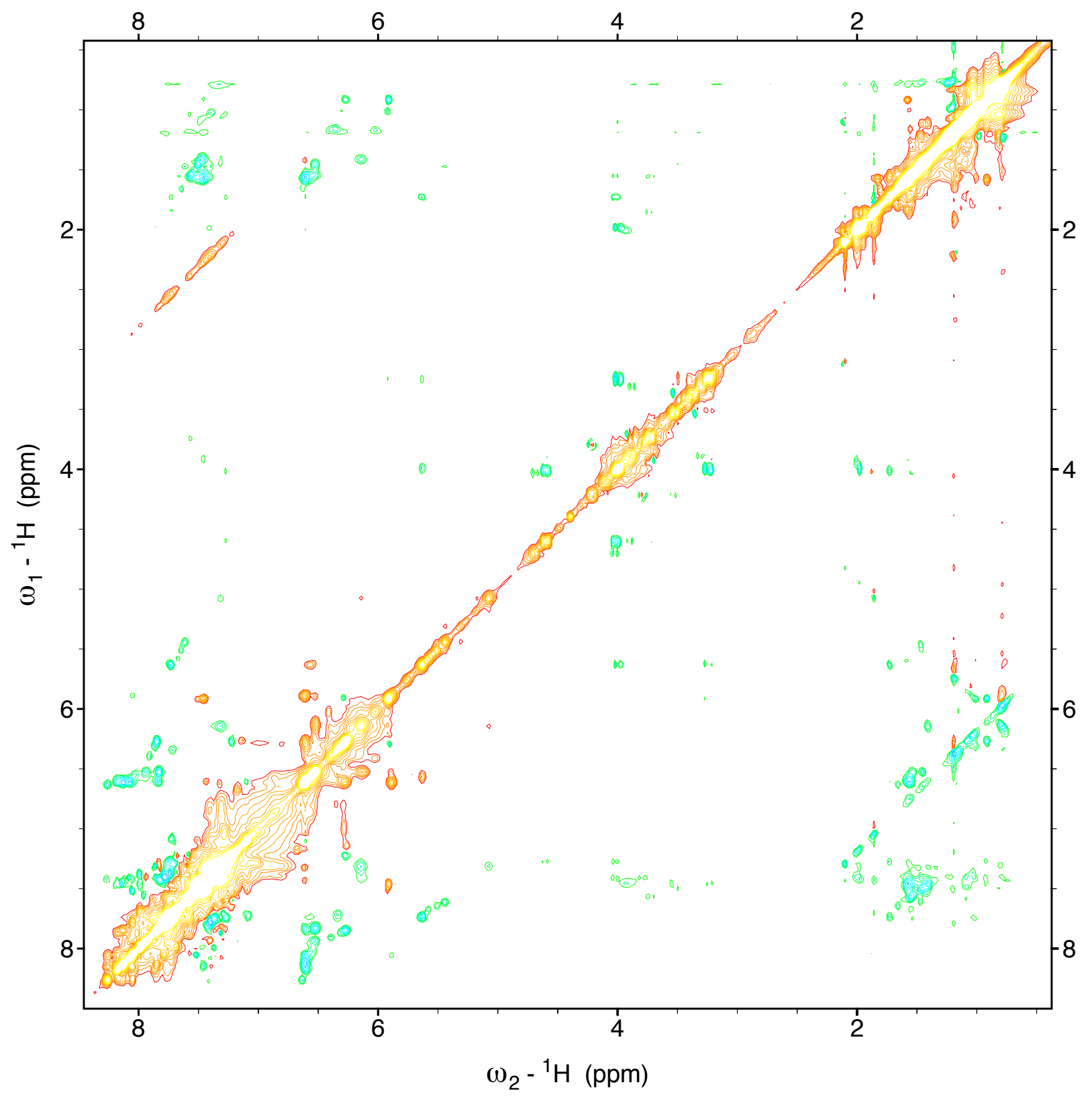




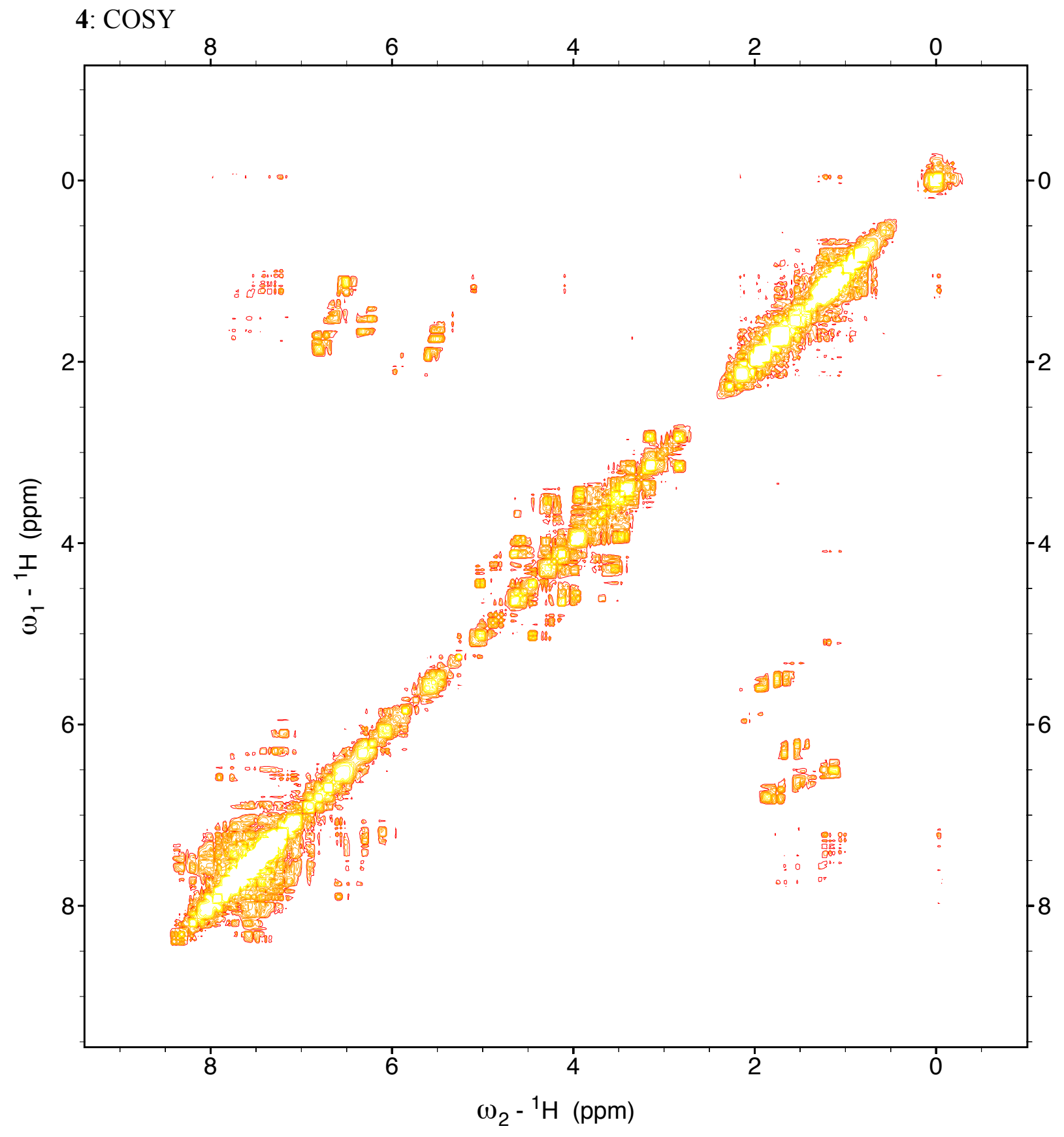

S- 26 


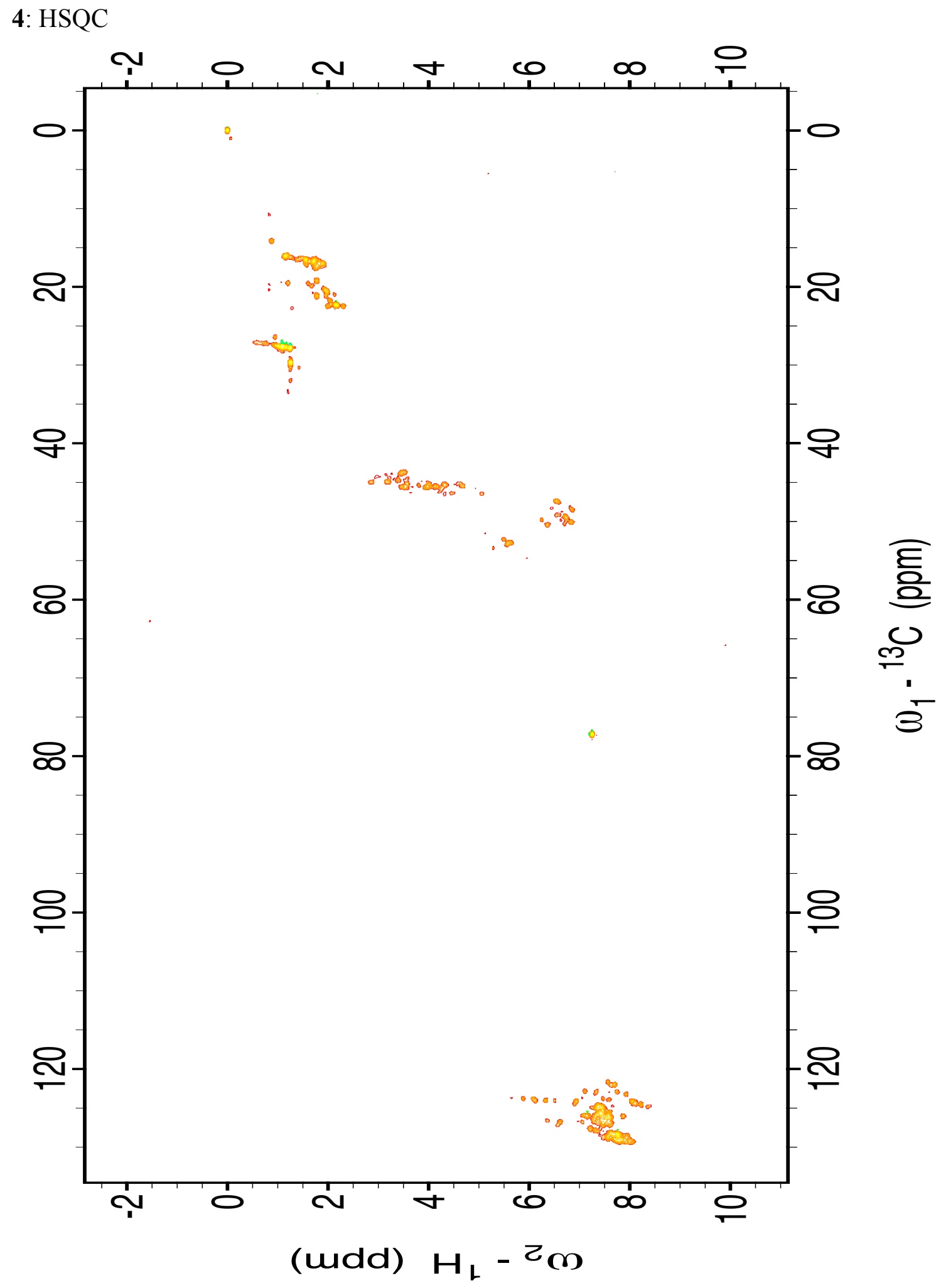


3: $\mathrm{HMBC}$

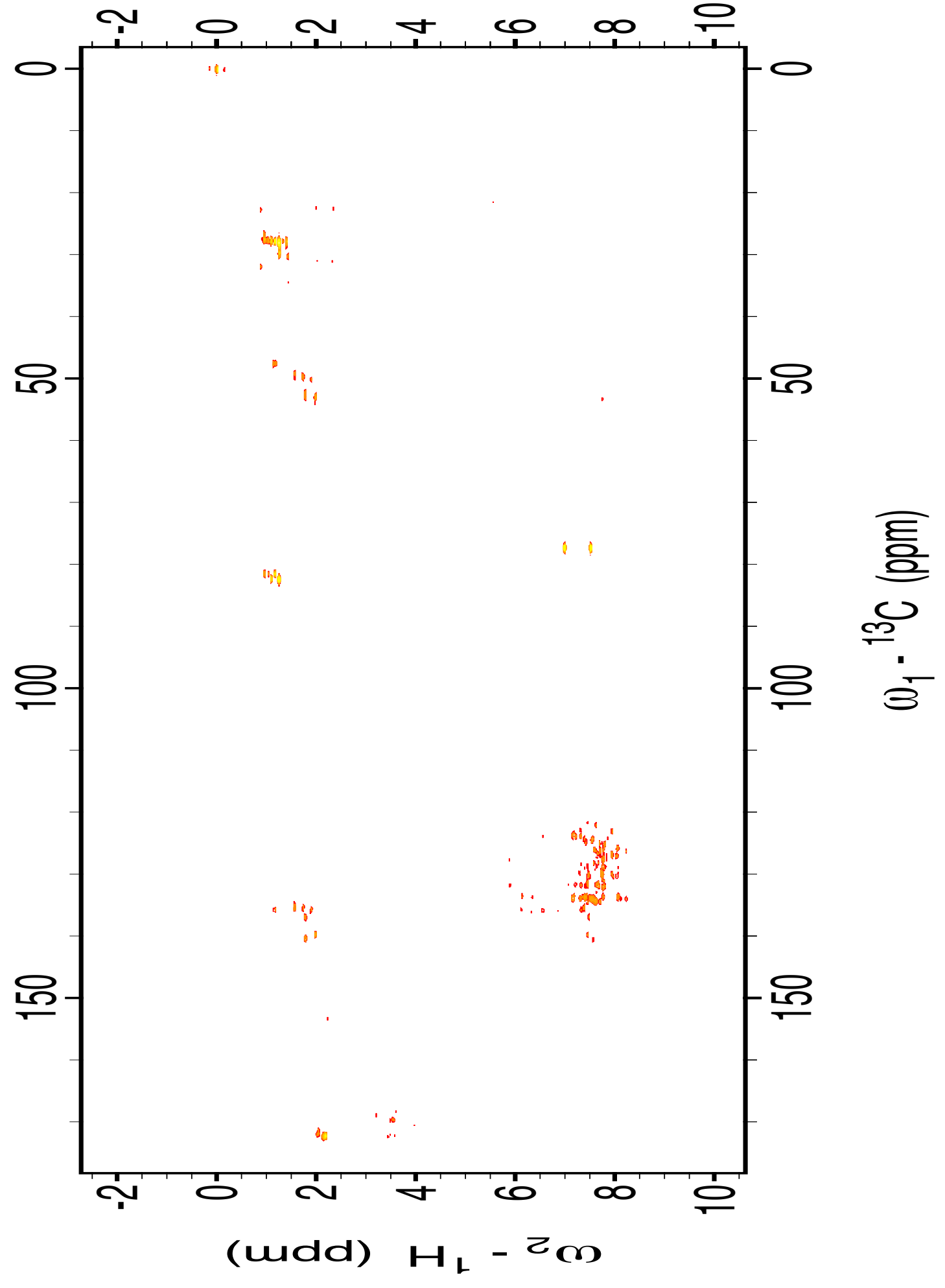




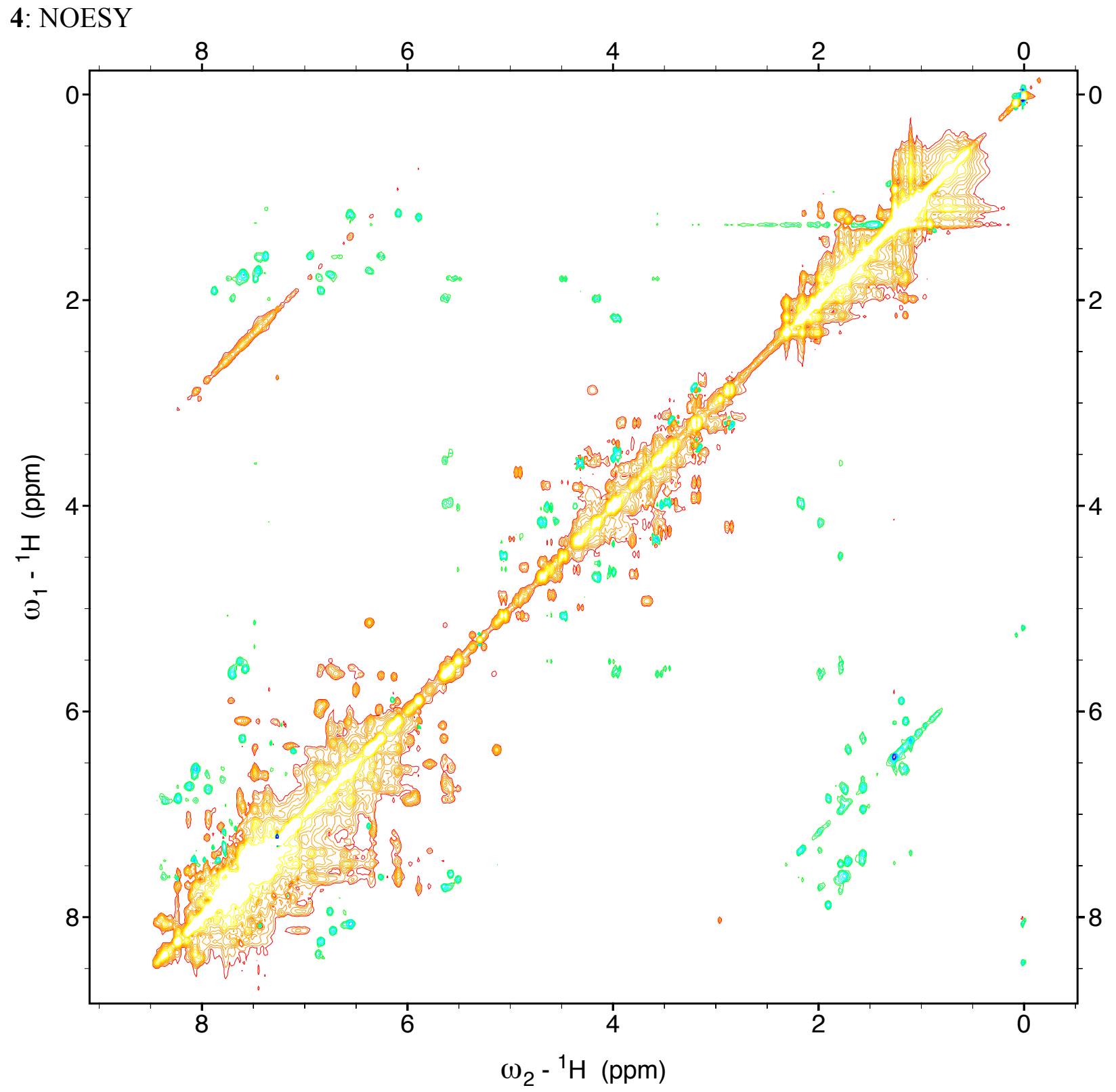




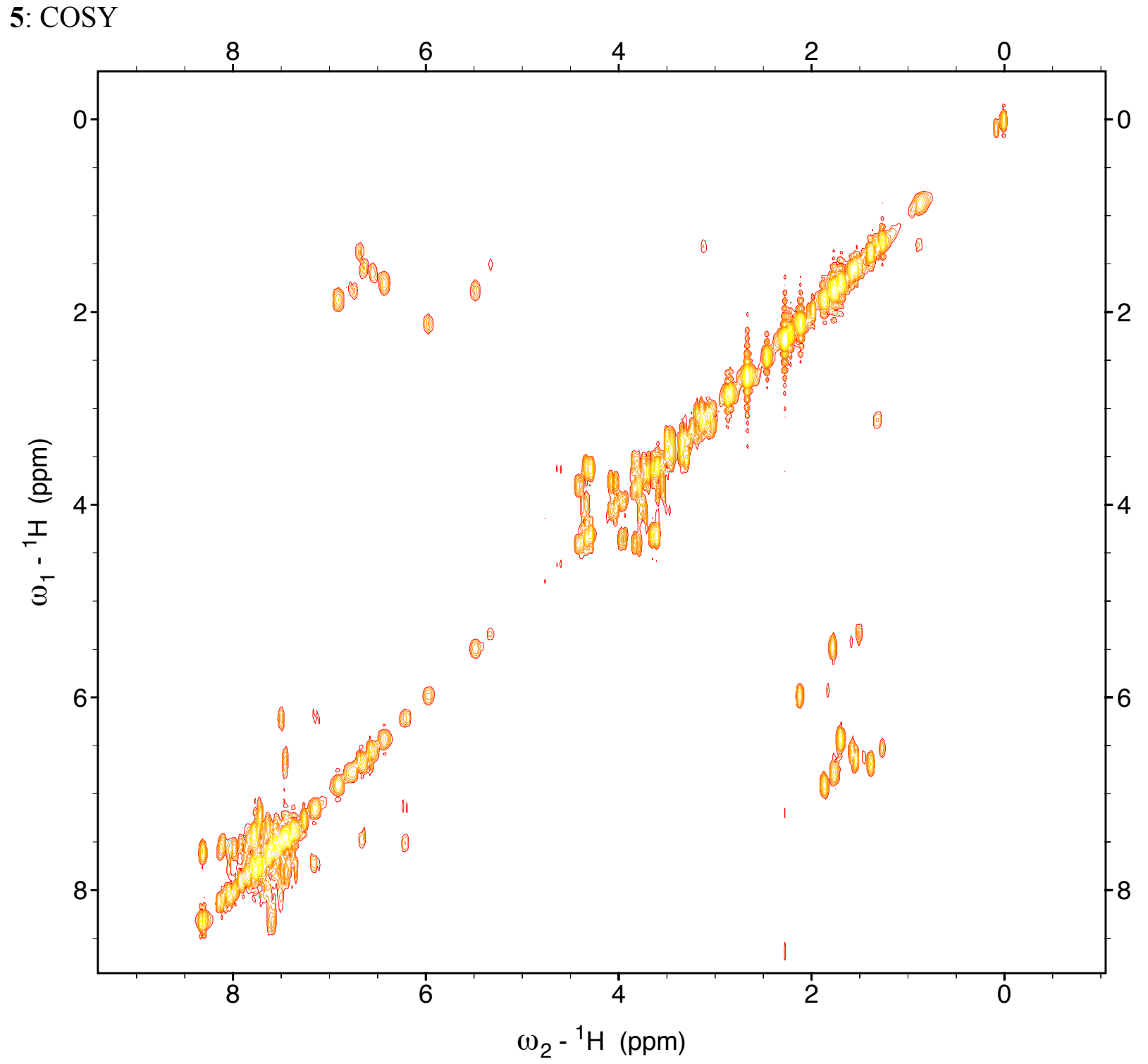




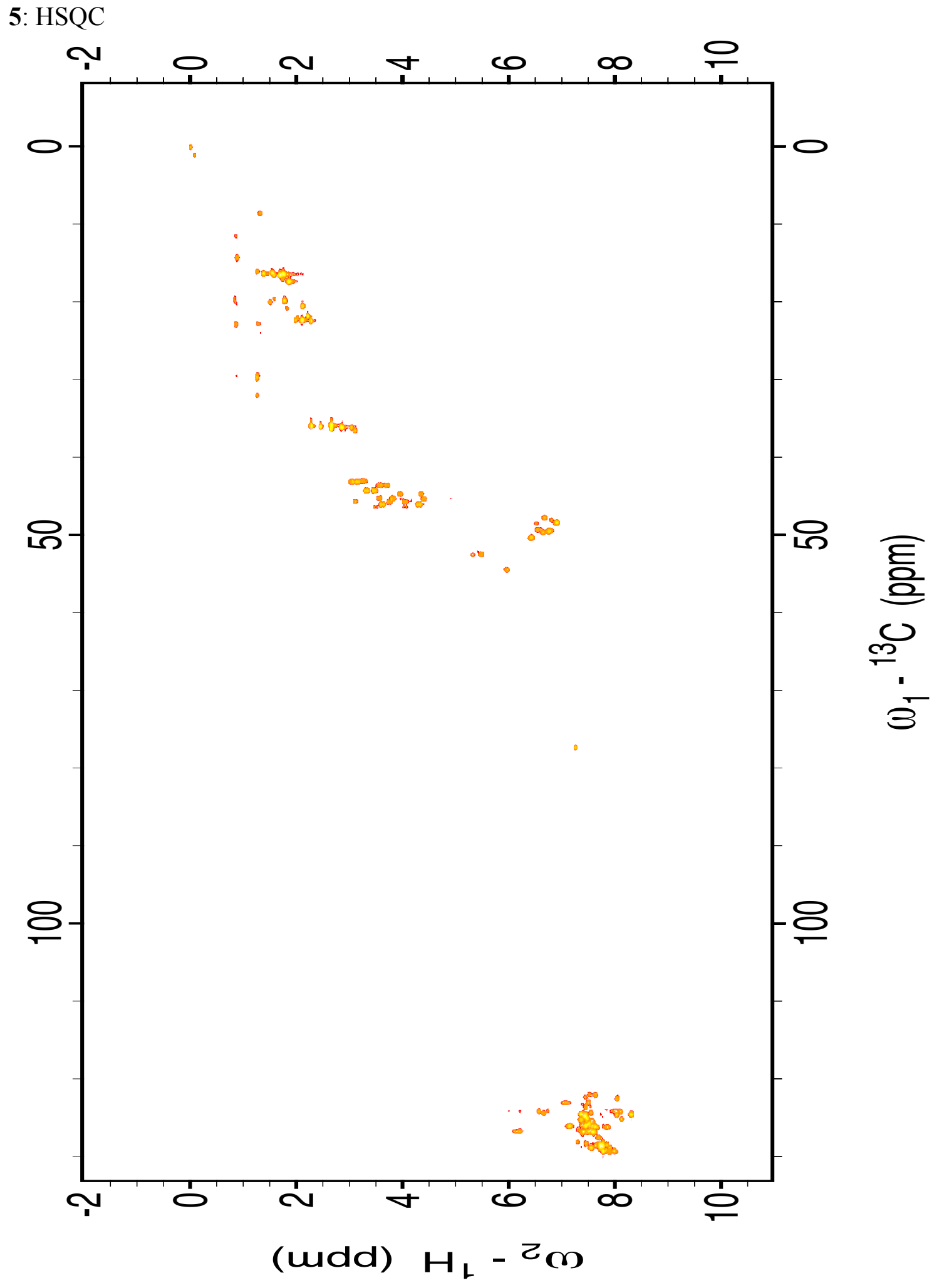




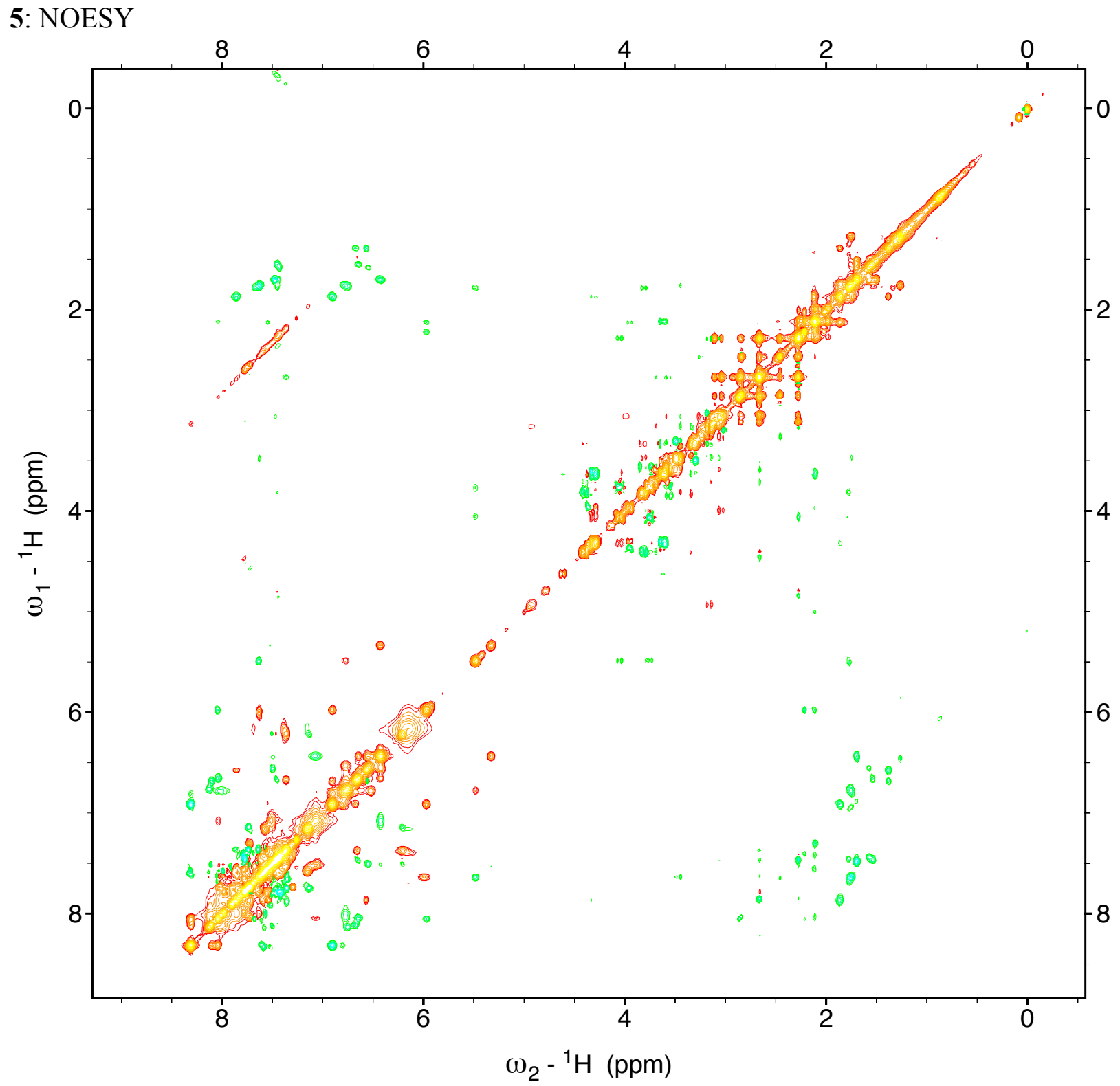

S- 32 


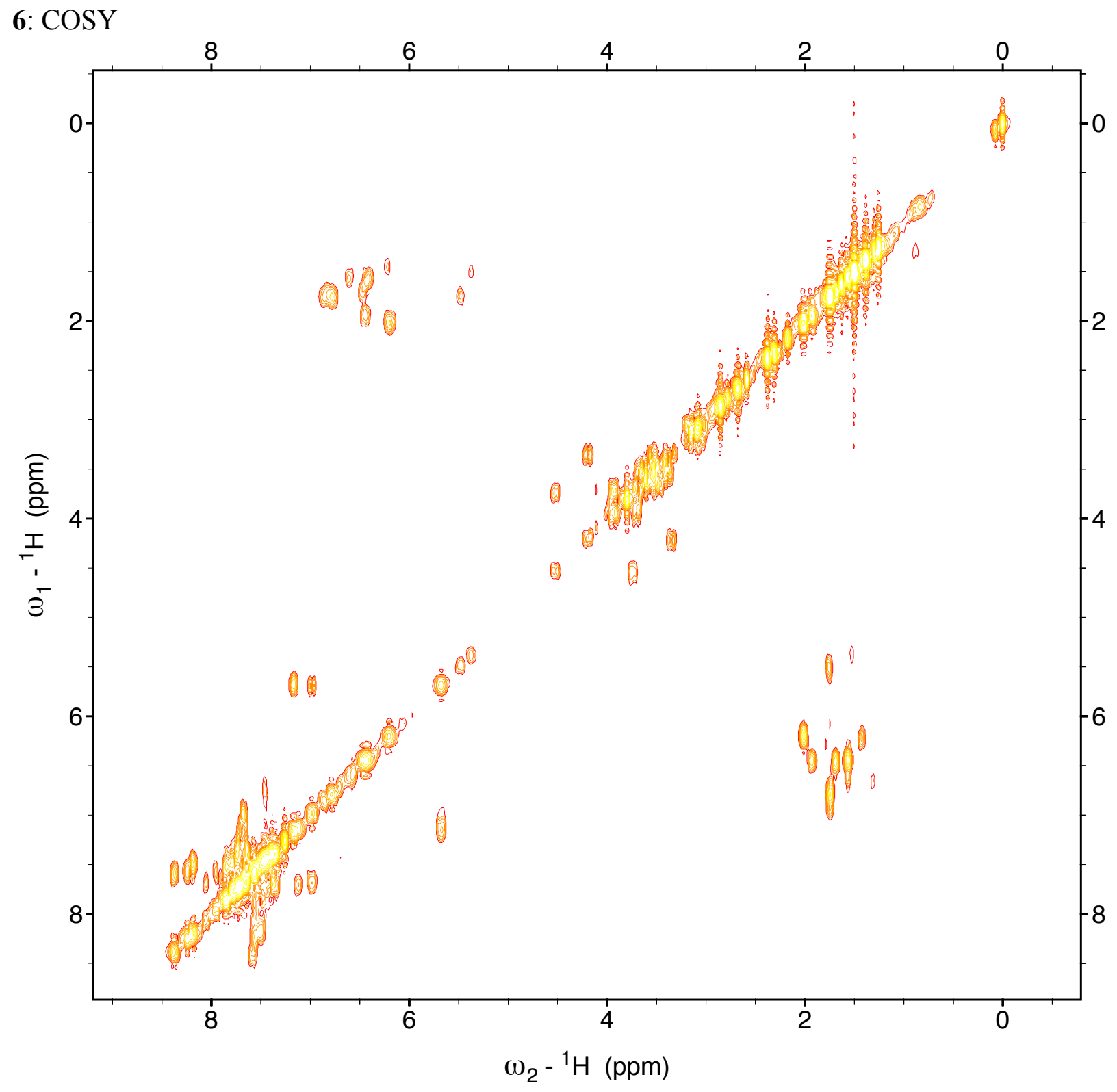

S- 33 


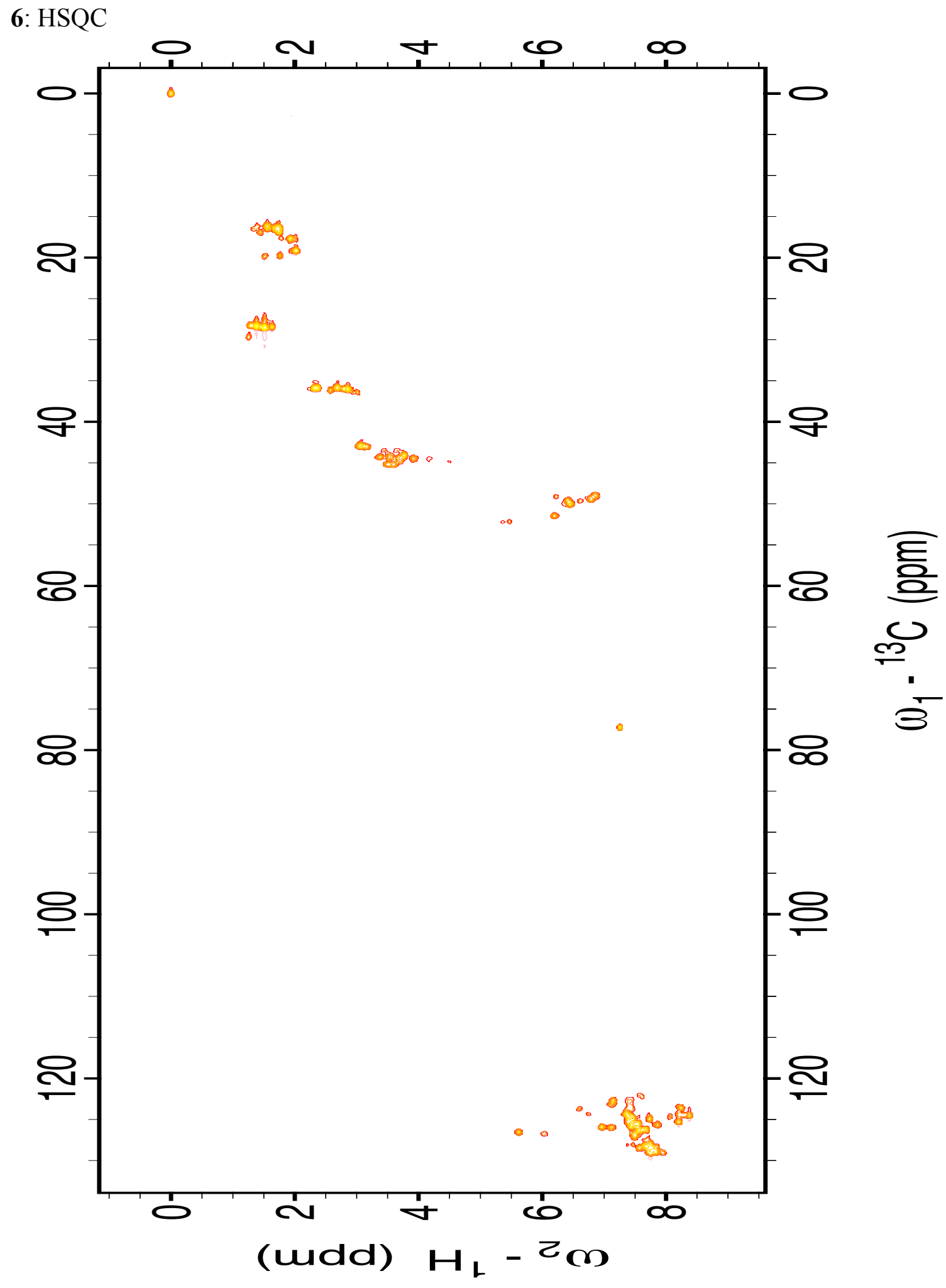




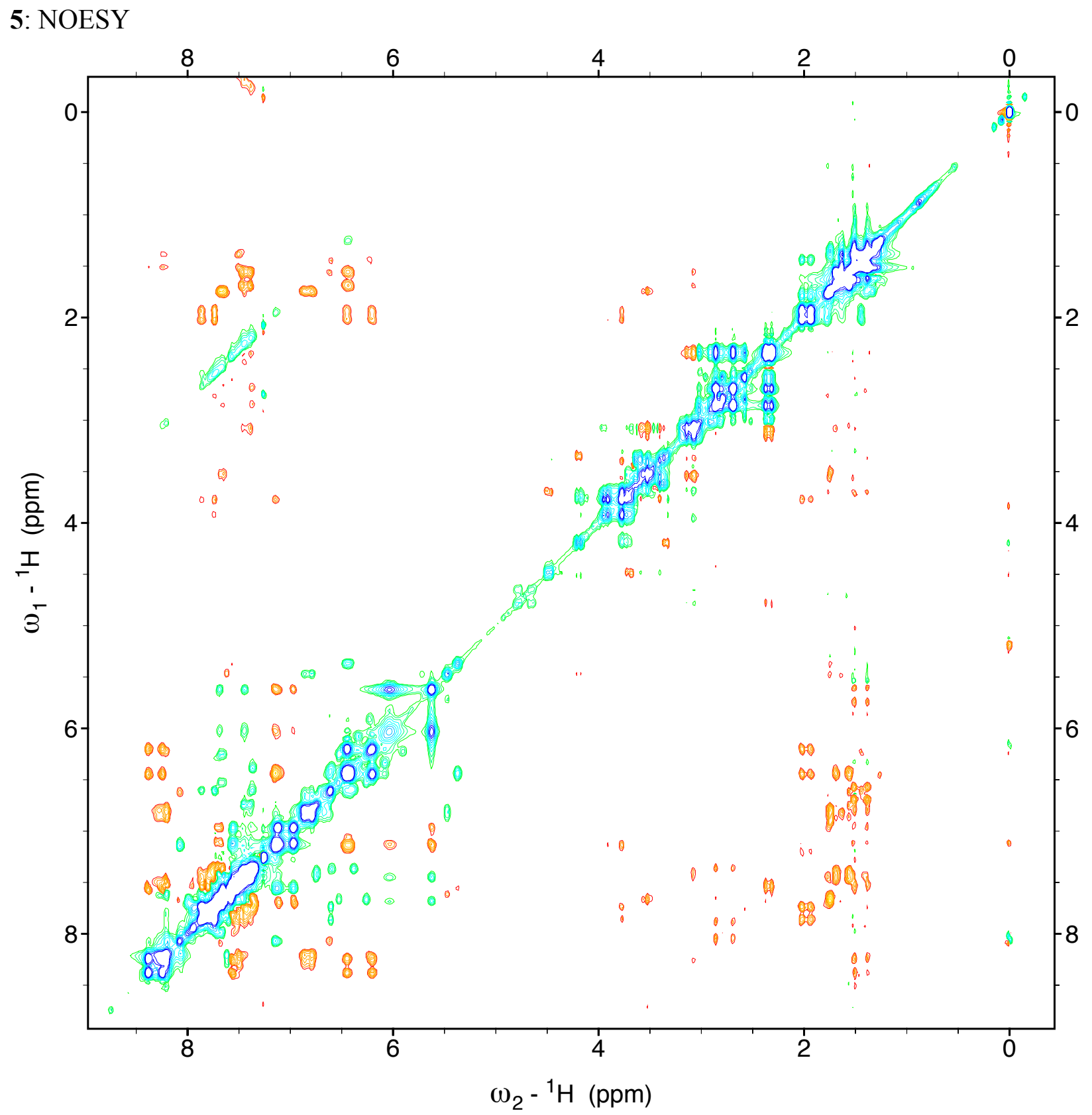

S- 35 


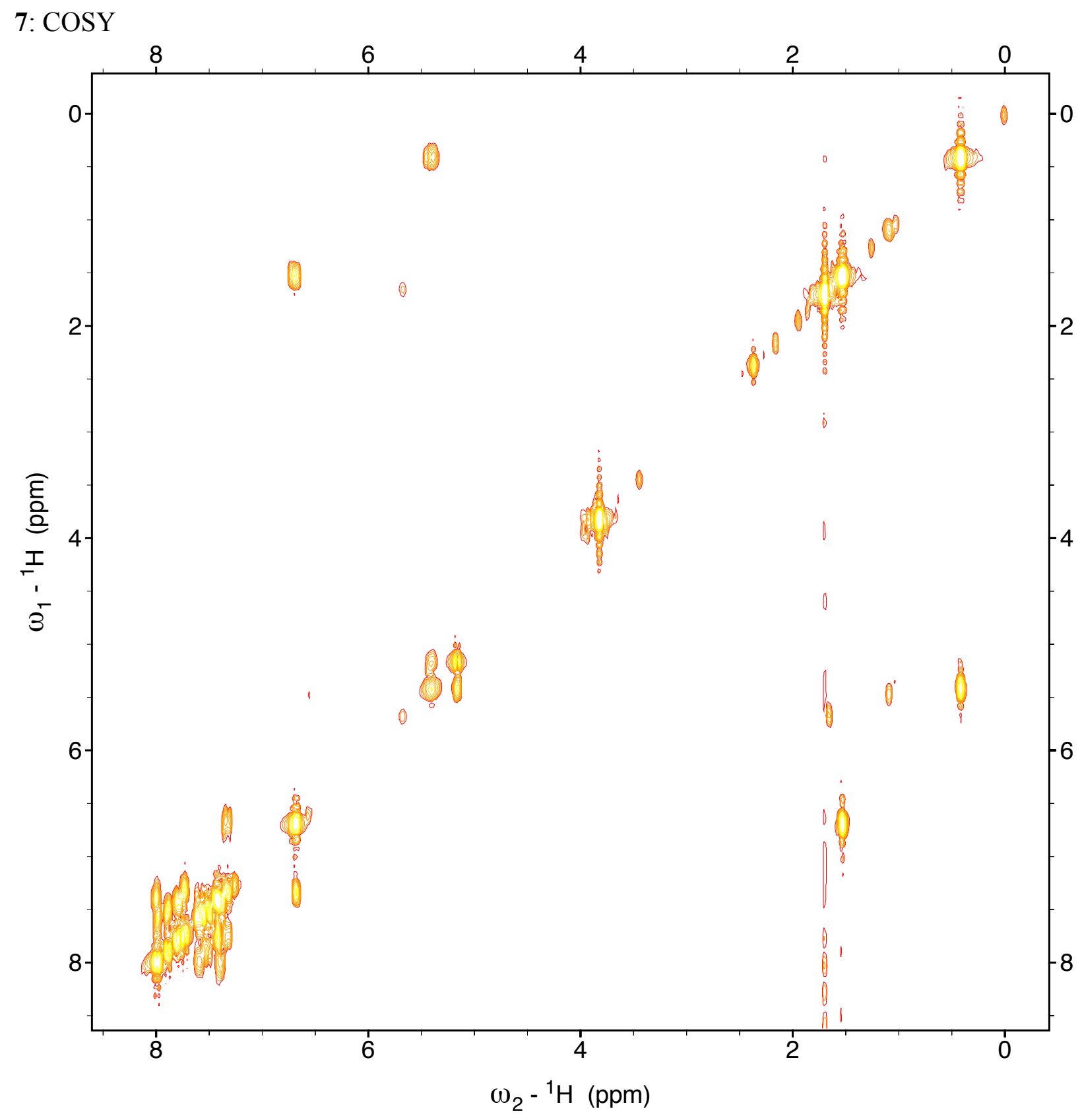

S- 36 


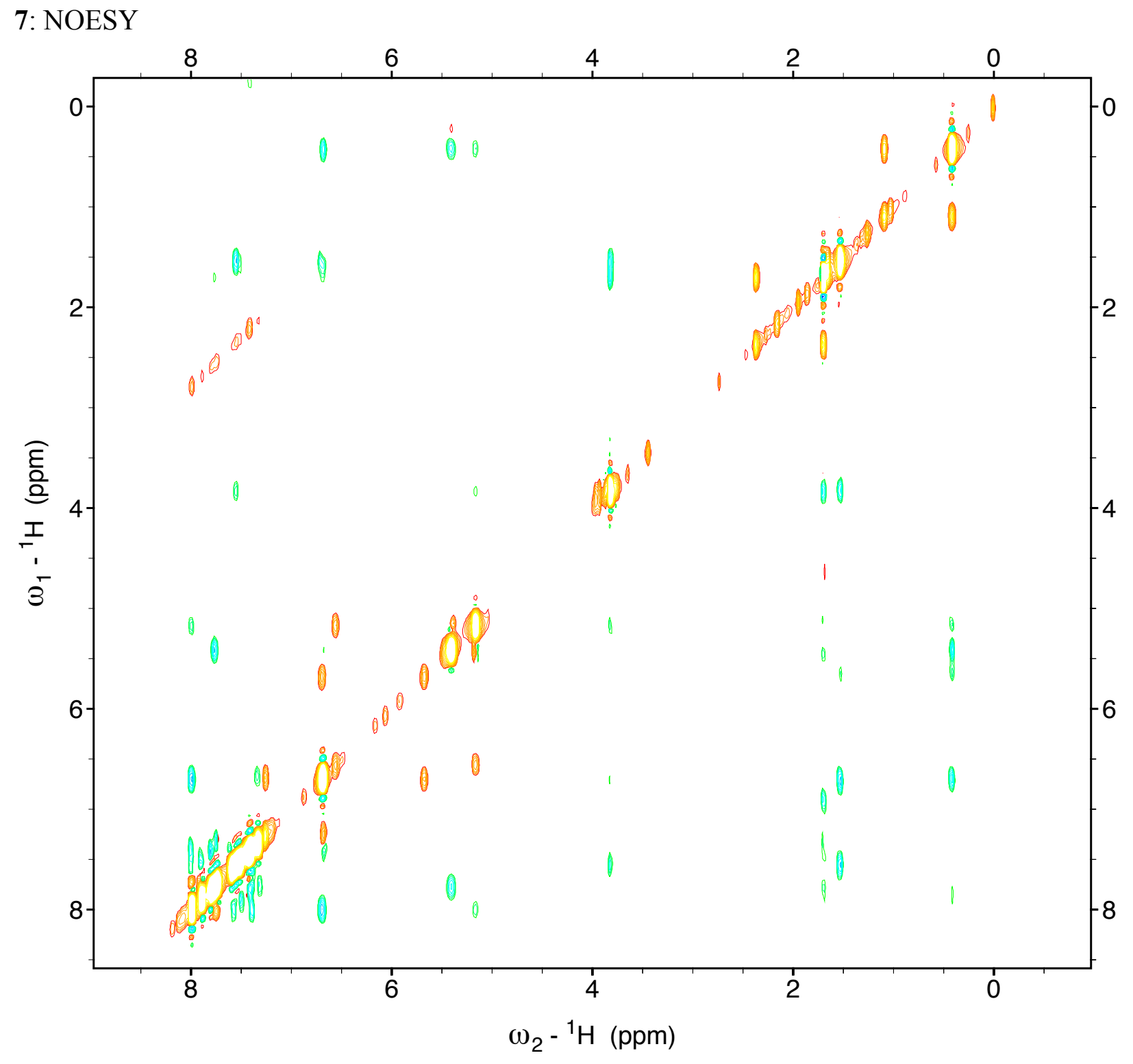

S- 37 


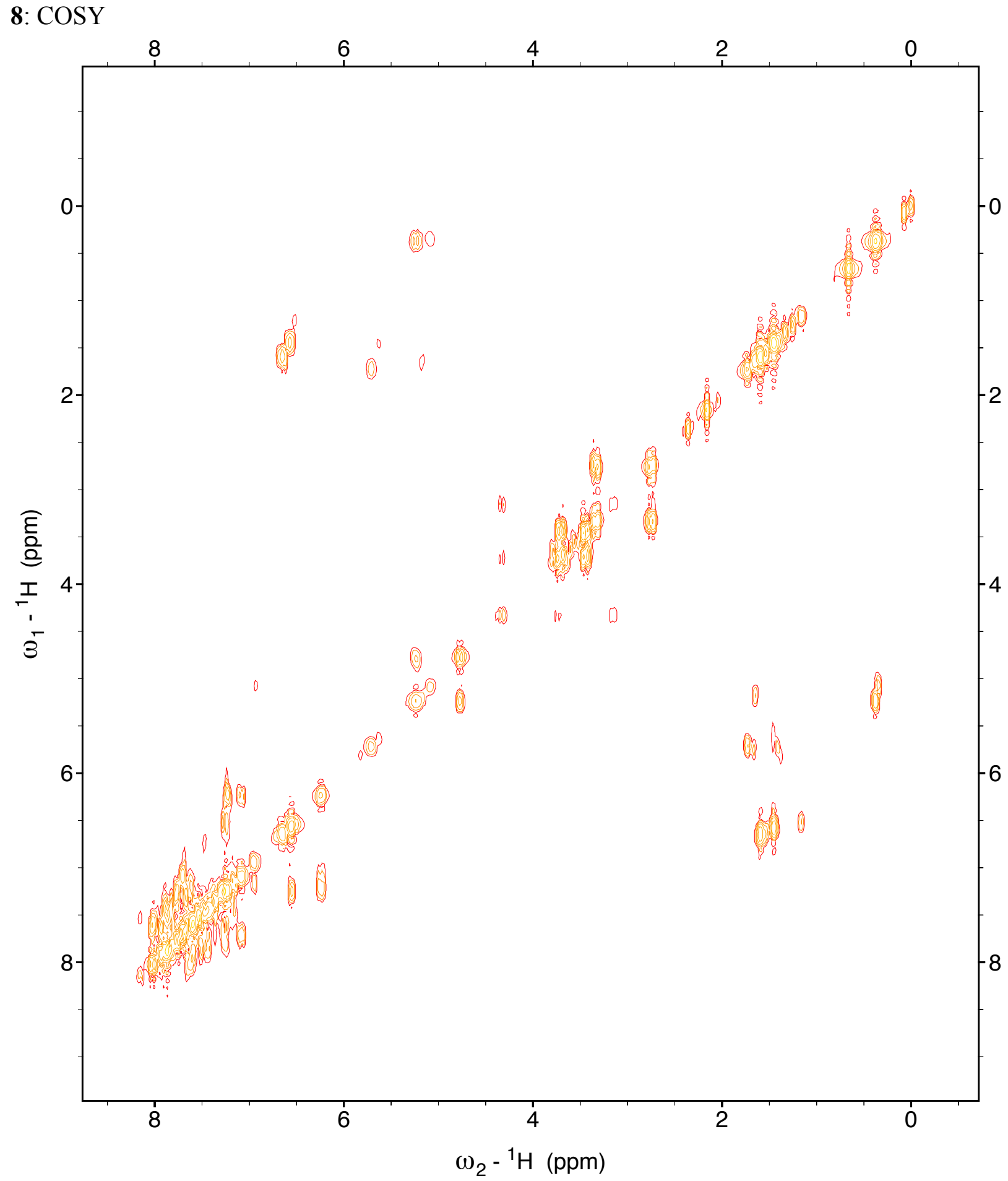

S- 38 


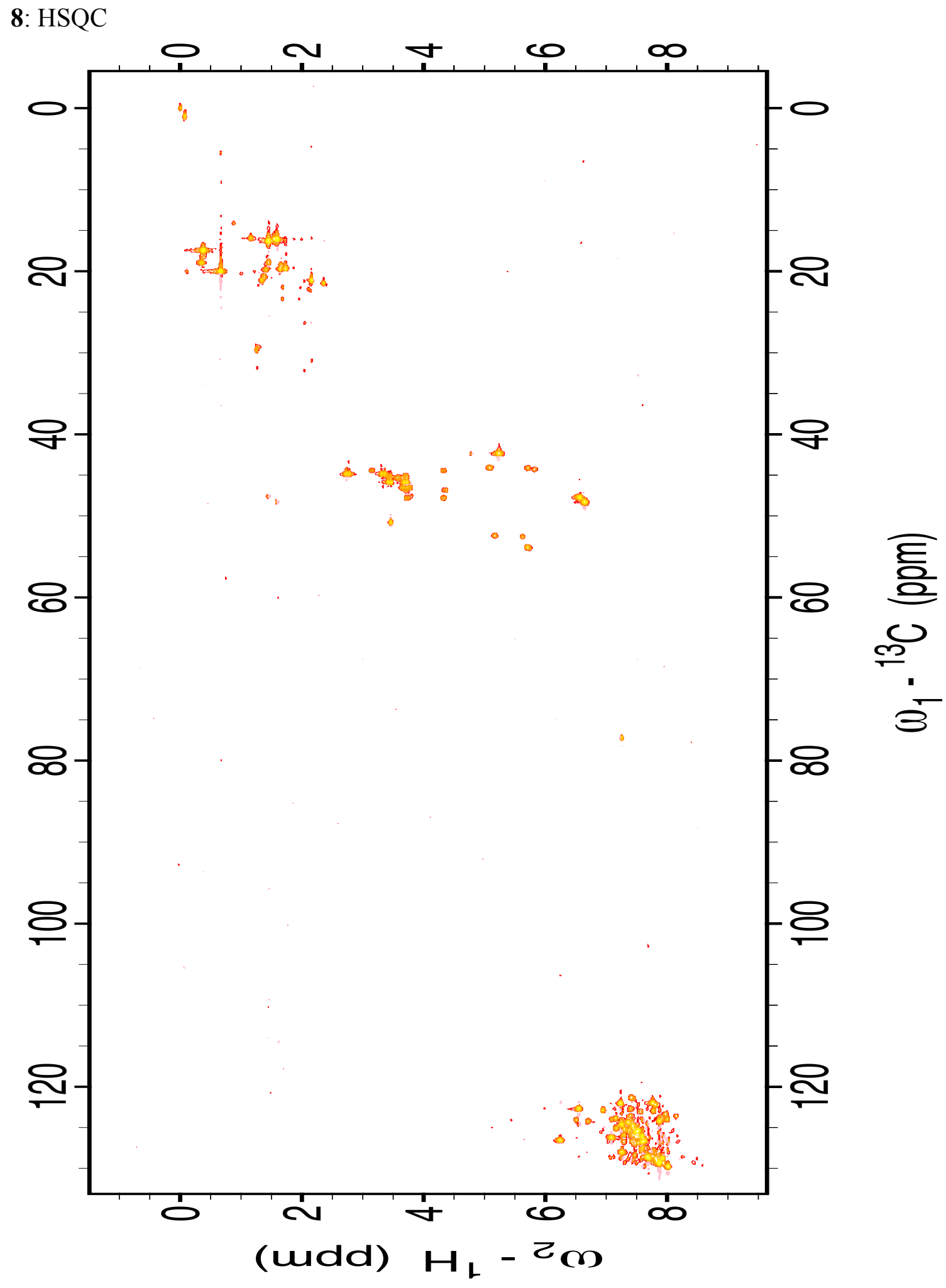




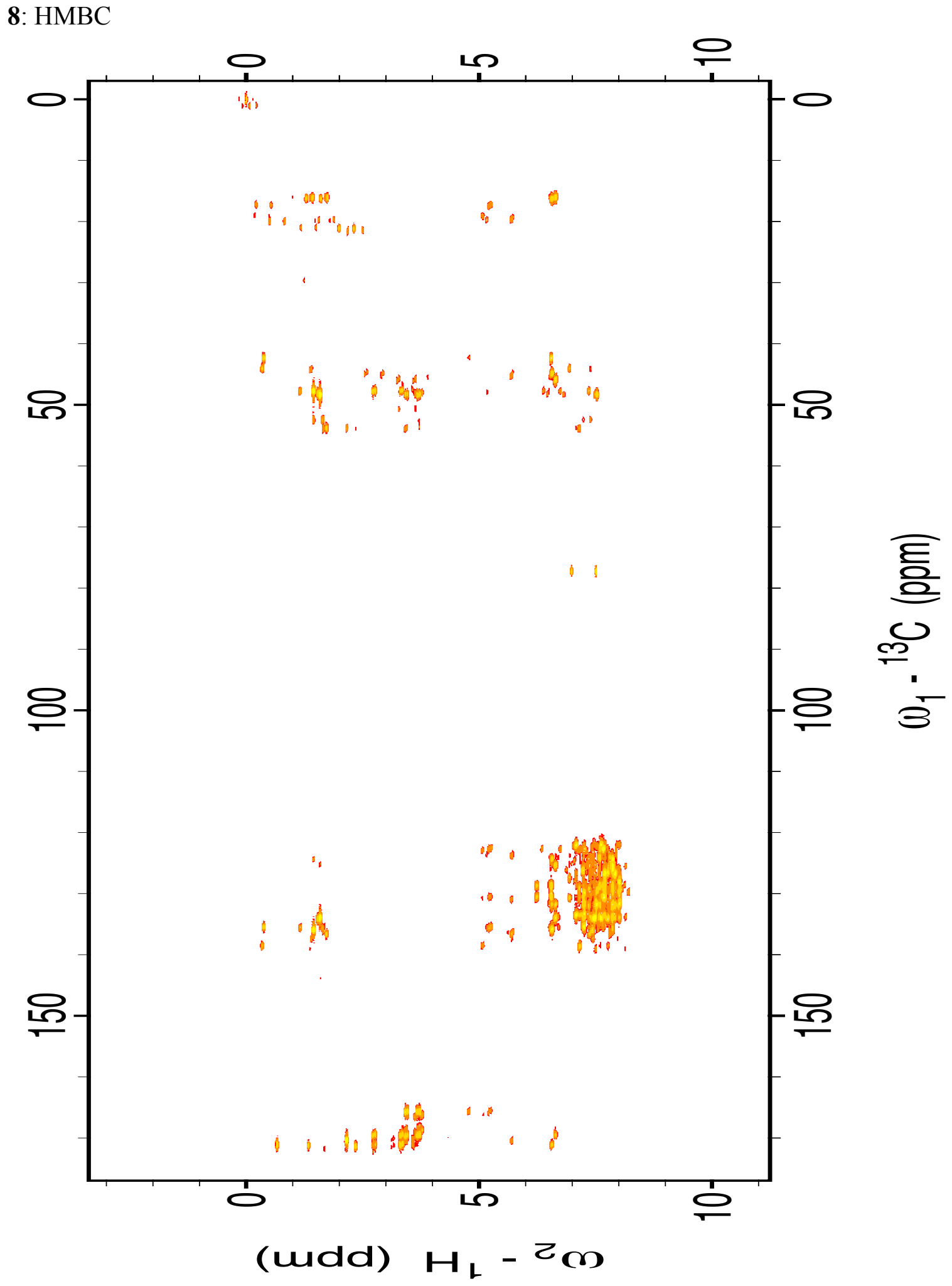




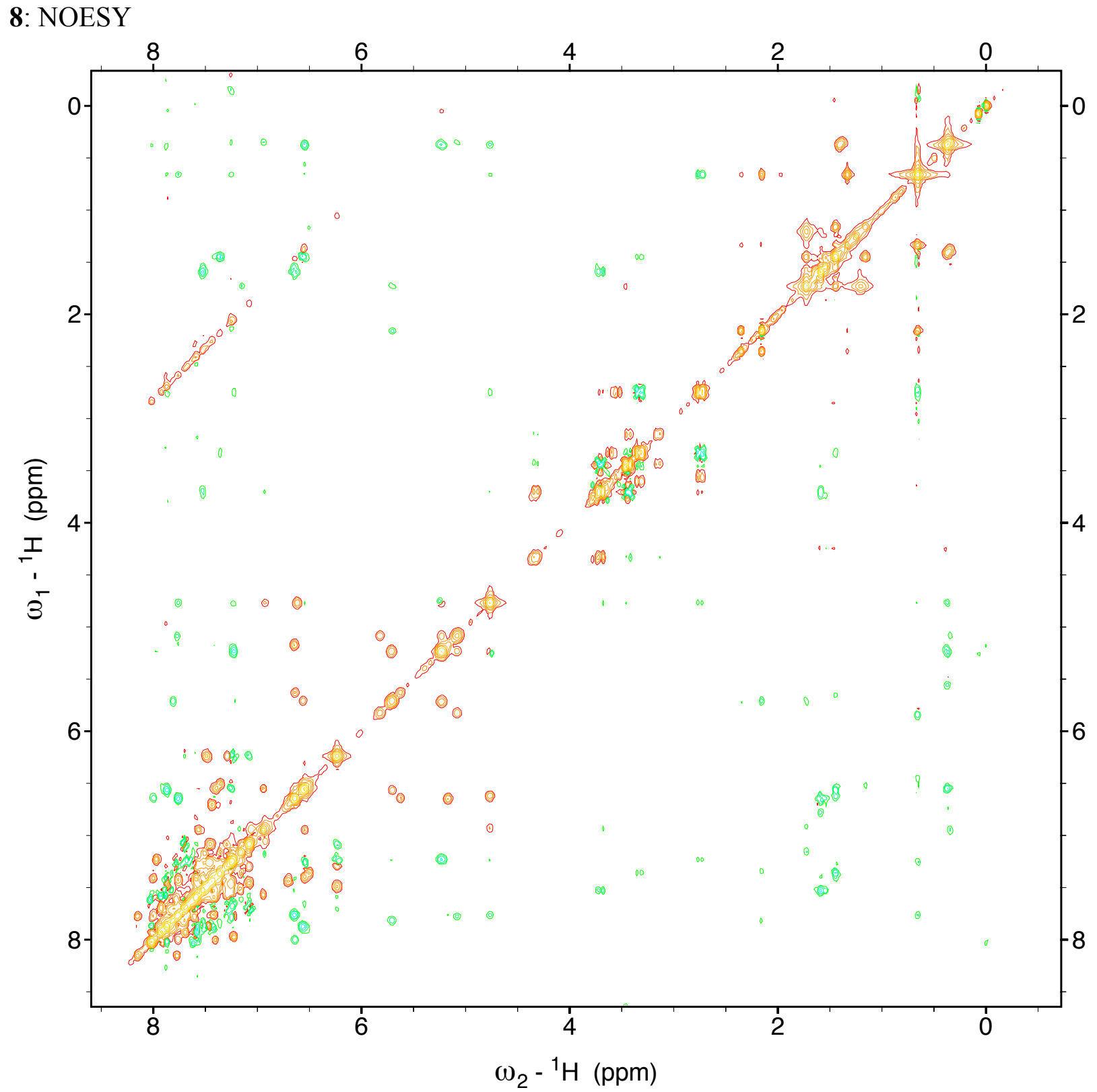

S- 41 


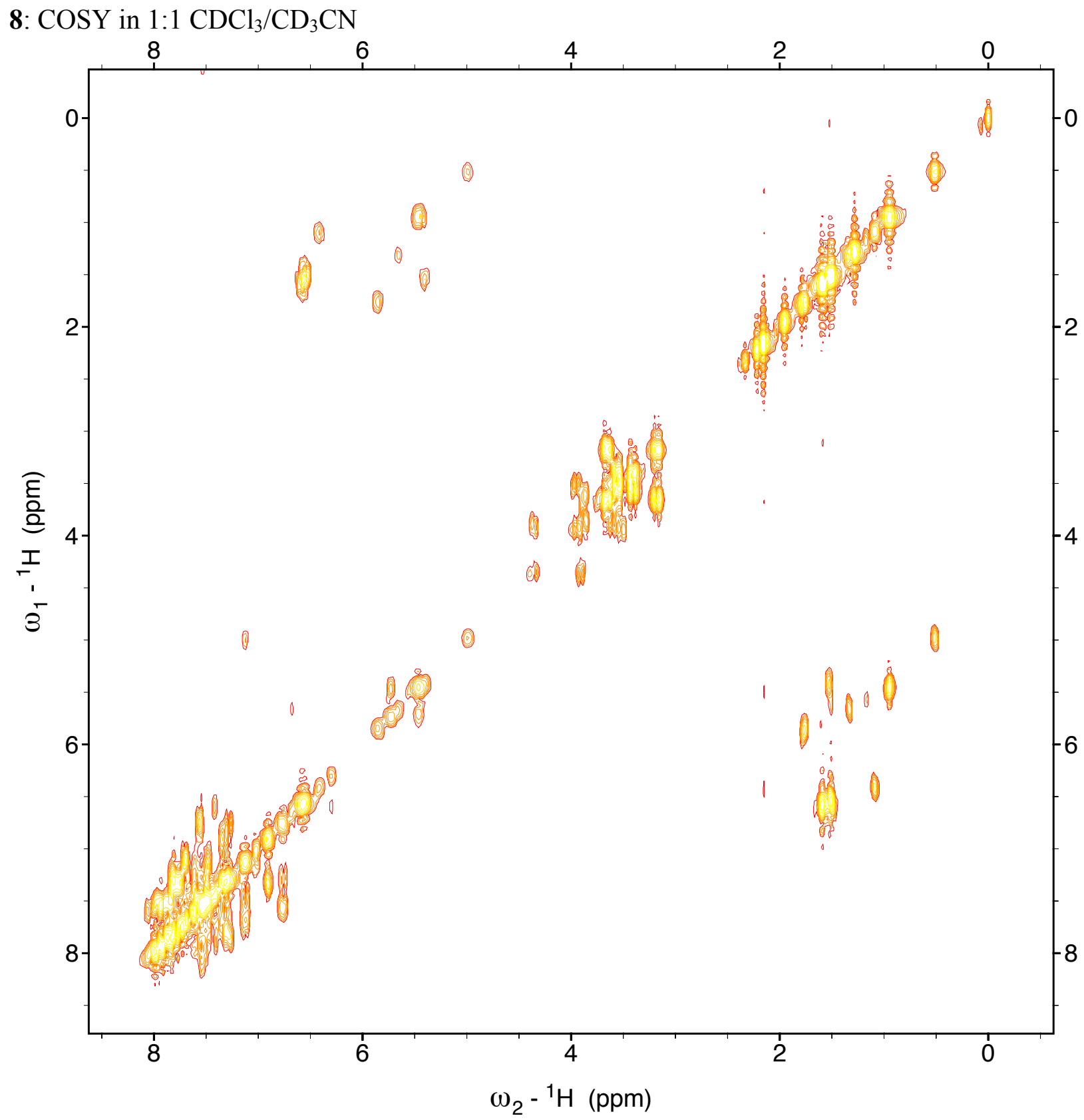

S- 42 


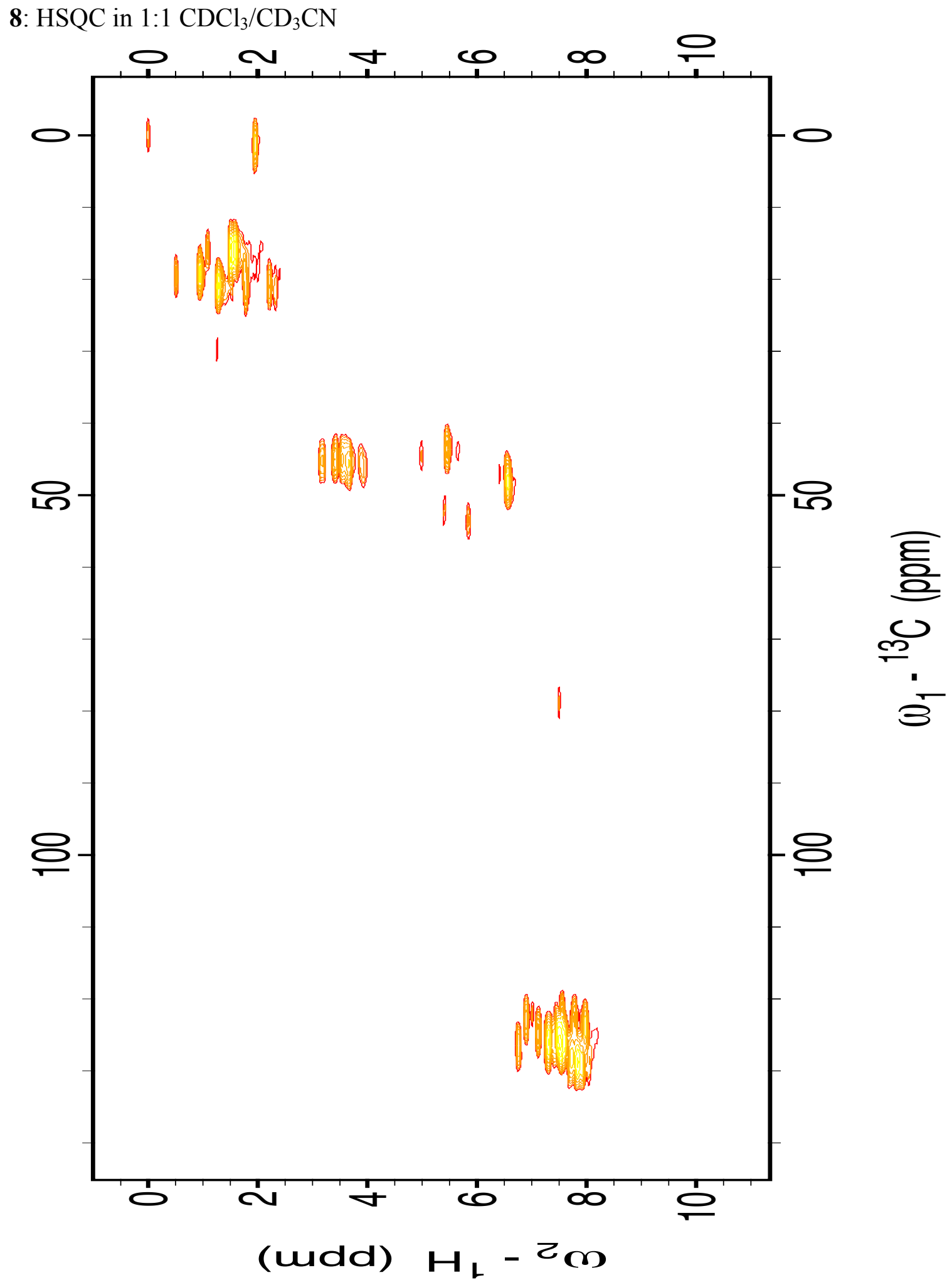


8: $\mathrm{HMBC}$ in $1: 1 \mathrm{CDCl}_{3} / \mathrm{CD}_{3} \mathrm{CN}$

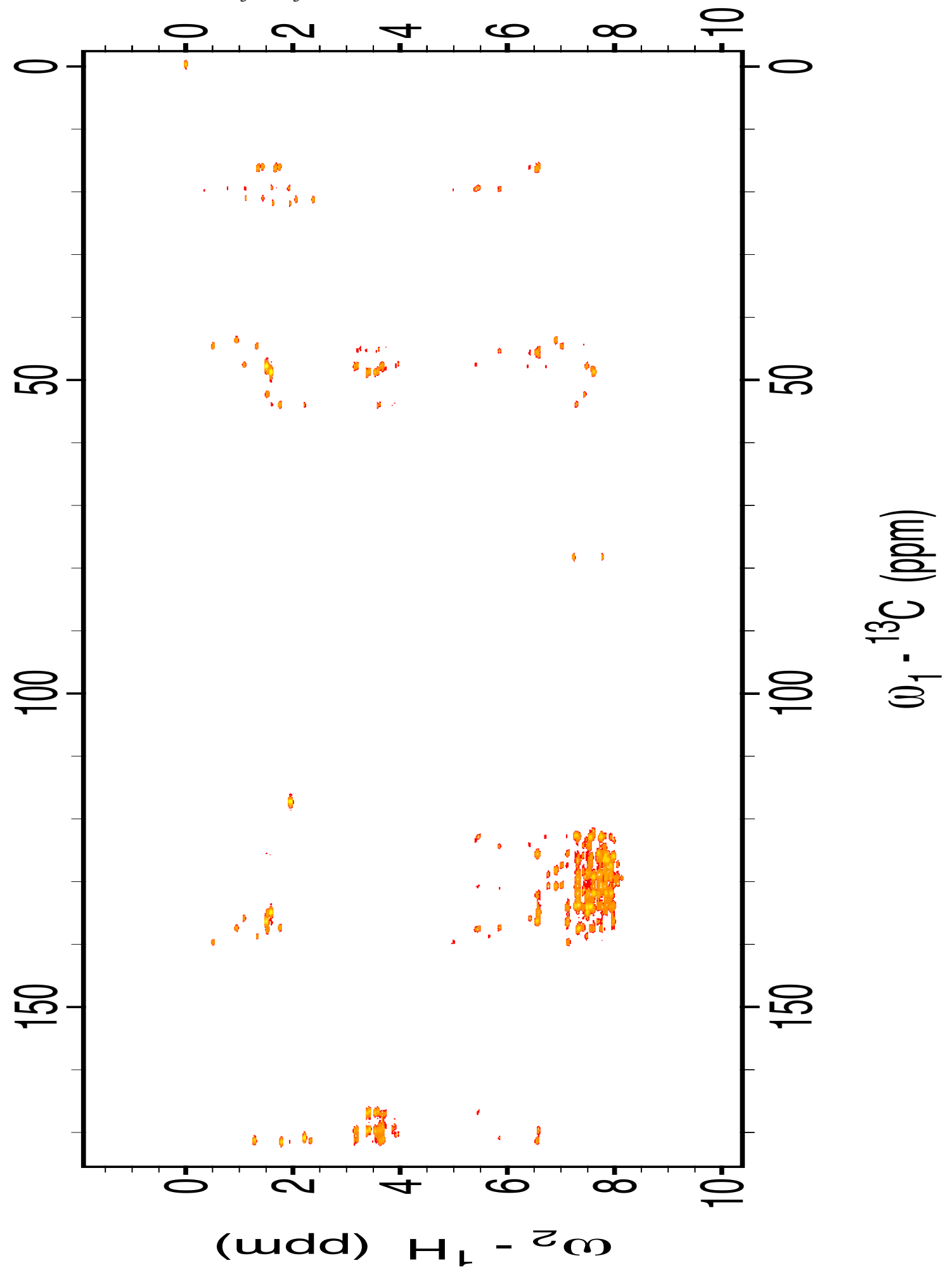


8: NOESY in $1: 1 \mathrm{CDCl}_{3} / \mathrm{CD}_{3} \mathrm{CN}$

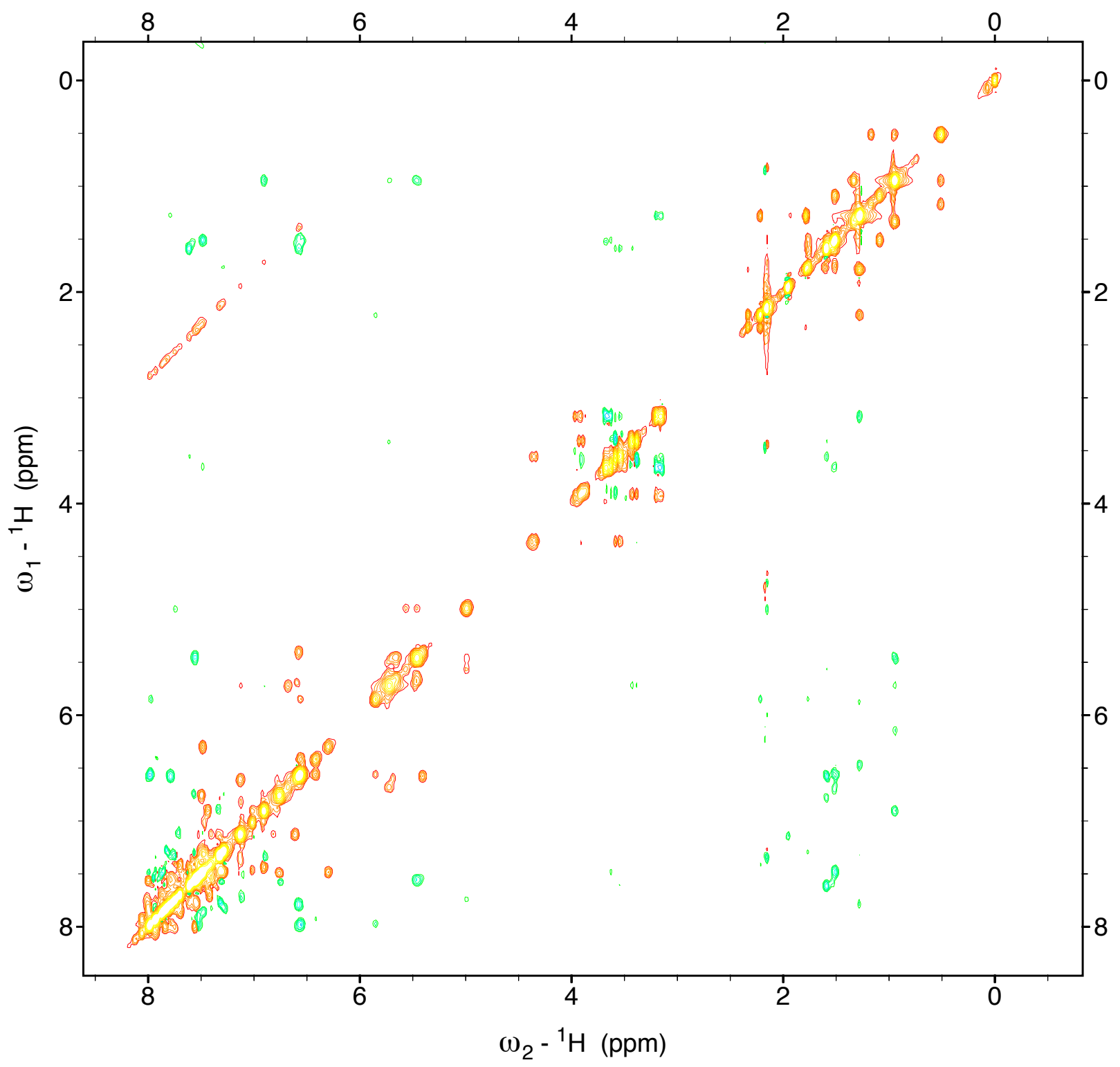




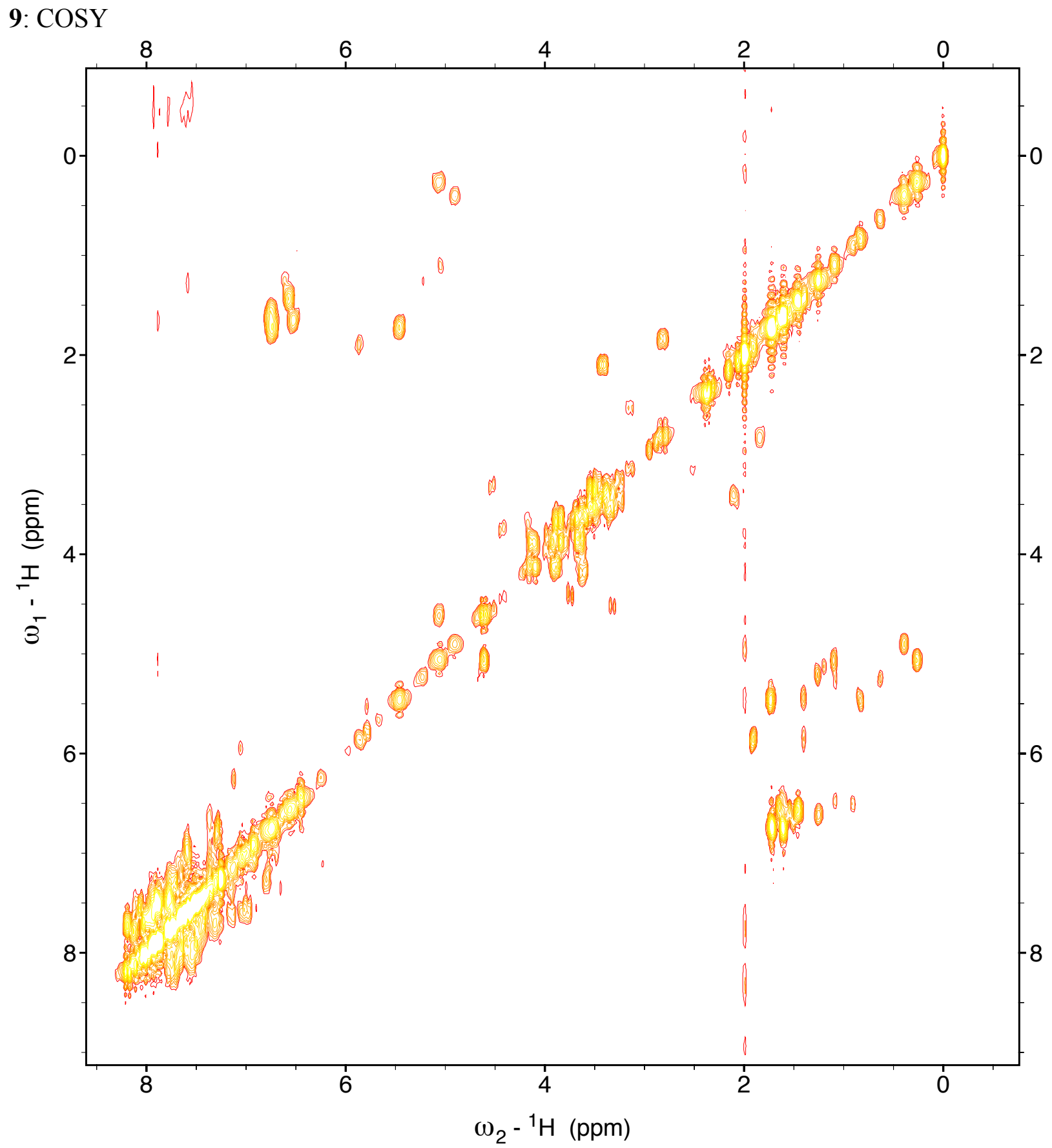




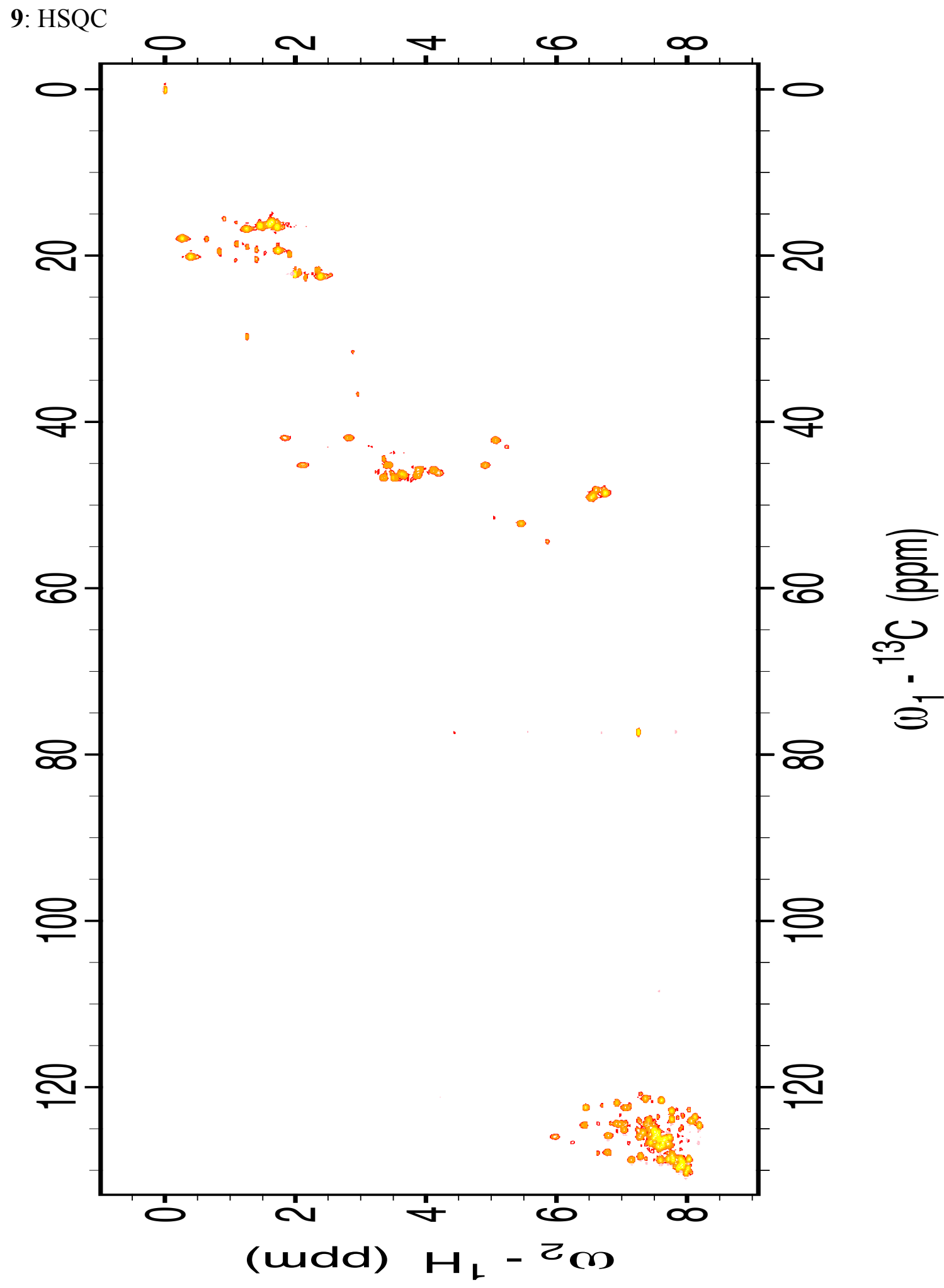




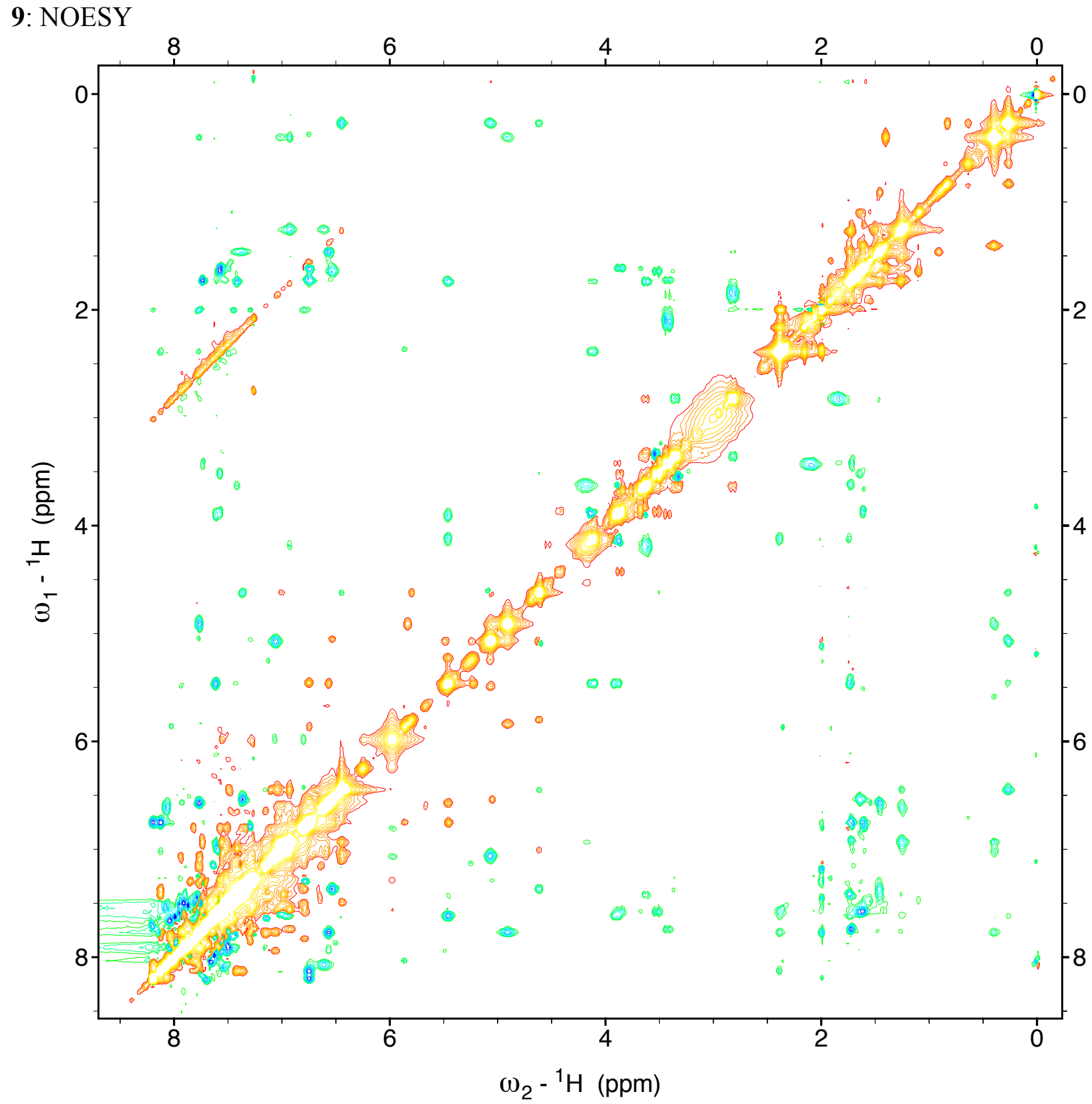




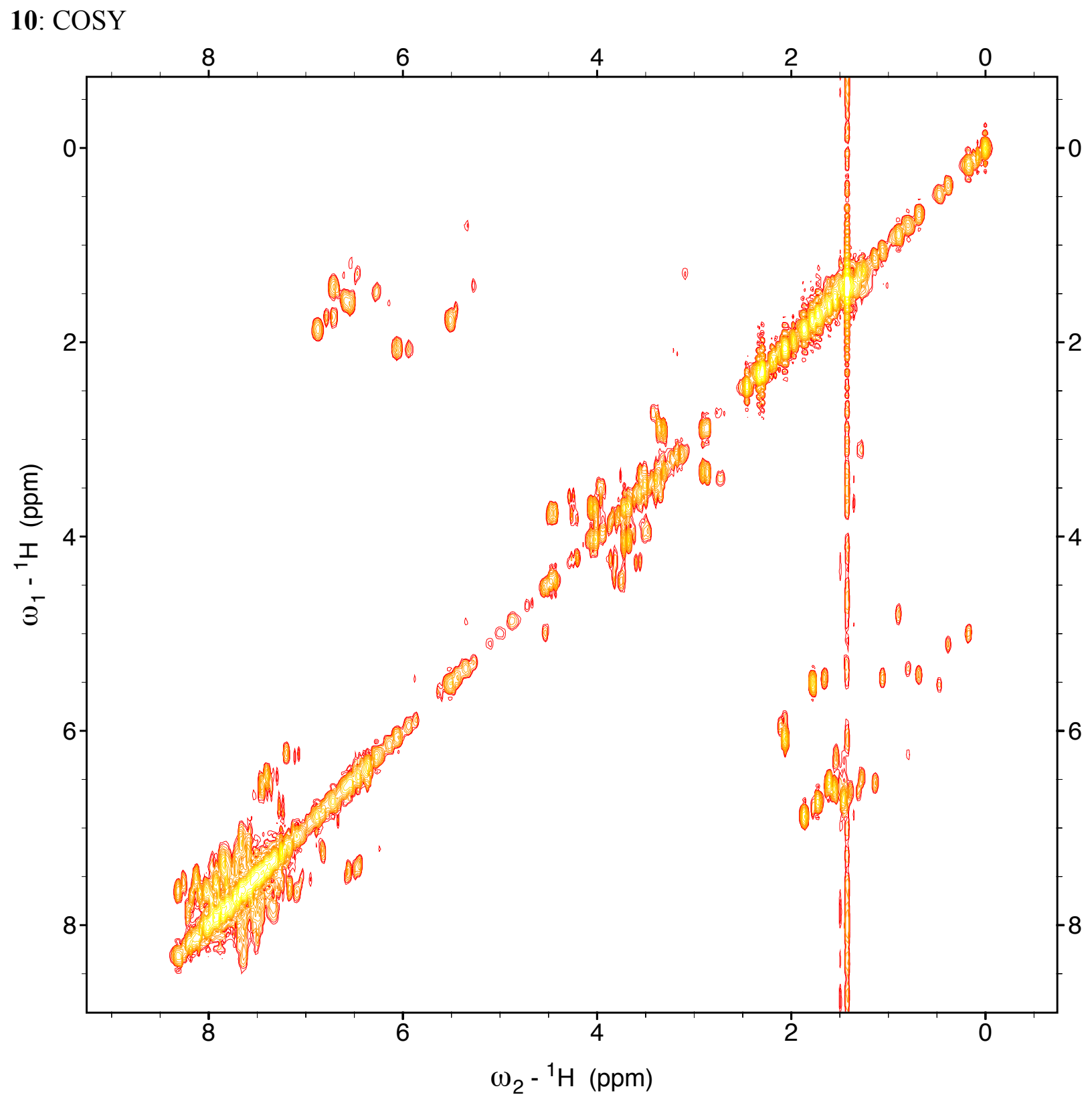


10: HSQC

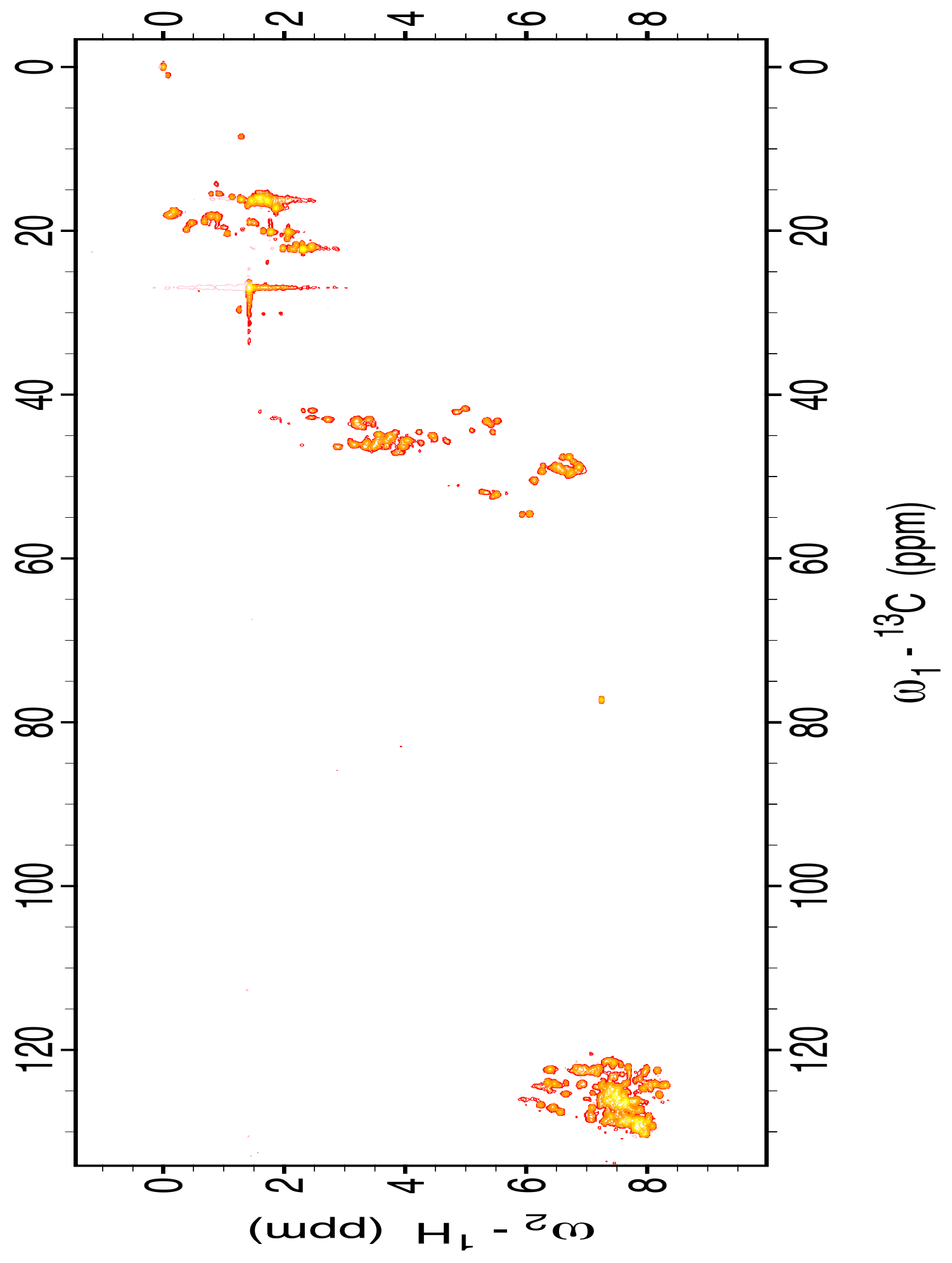




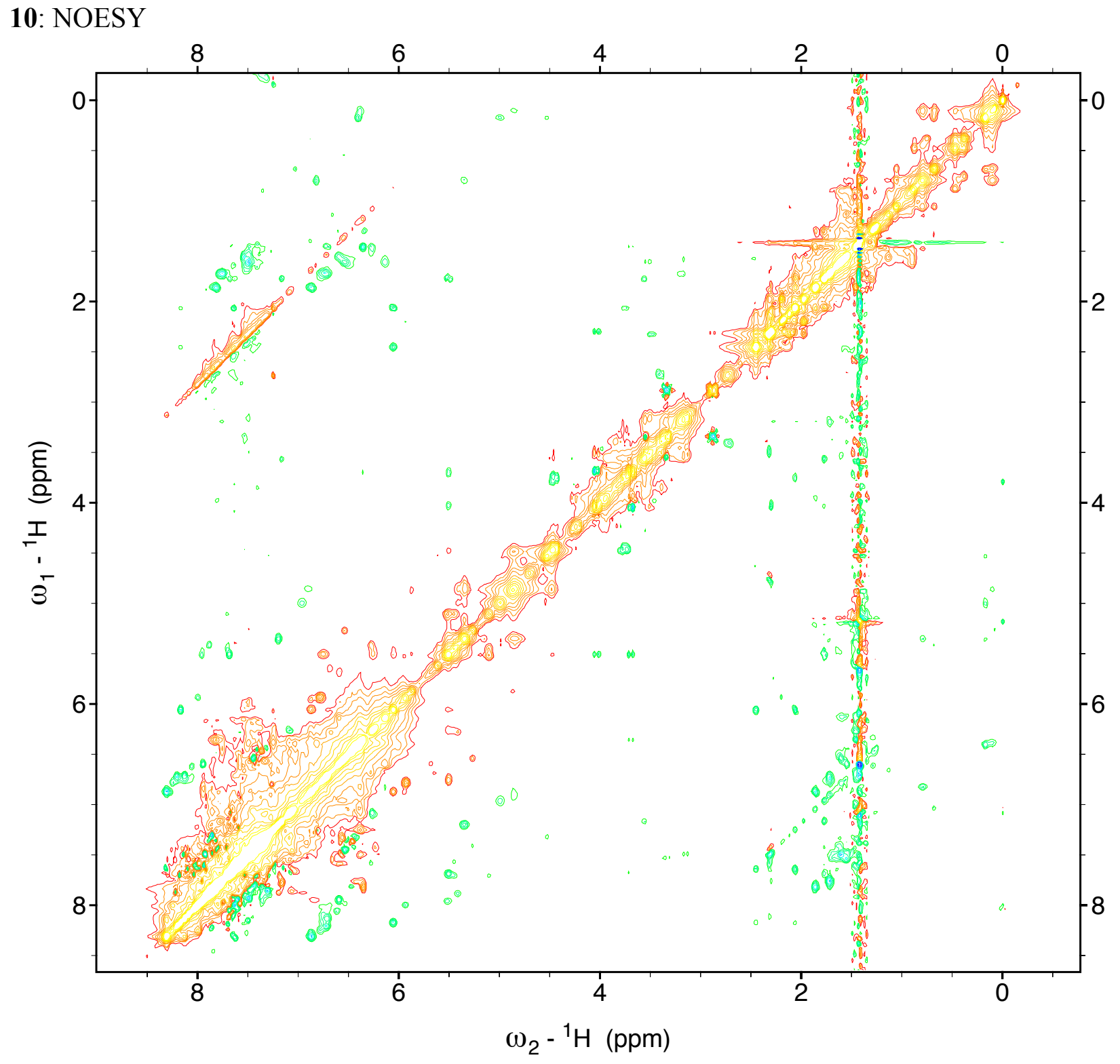

S- 51 


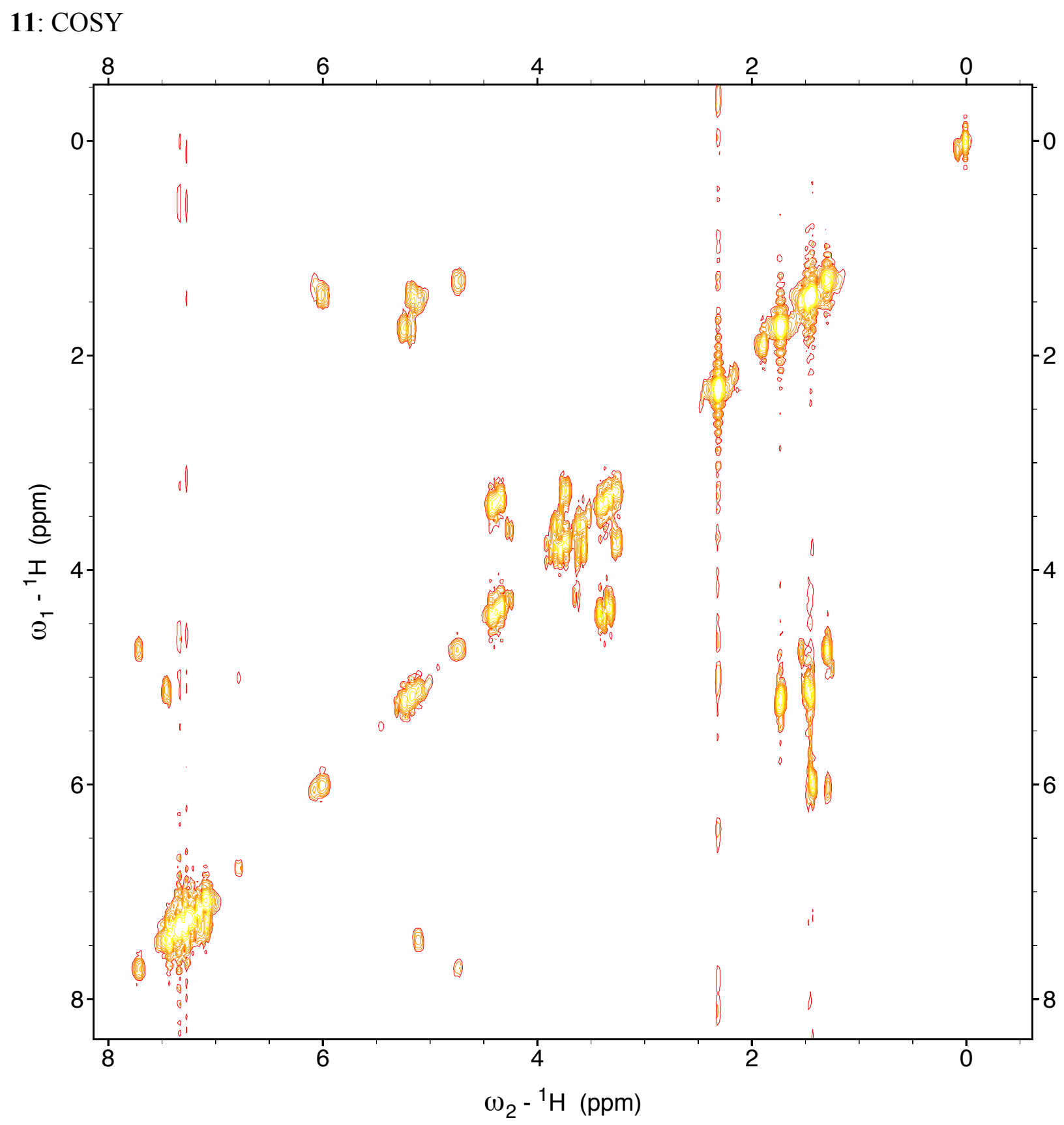

S- 52 


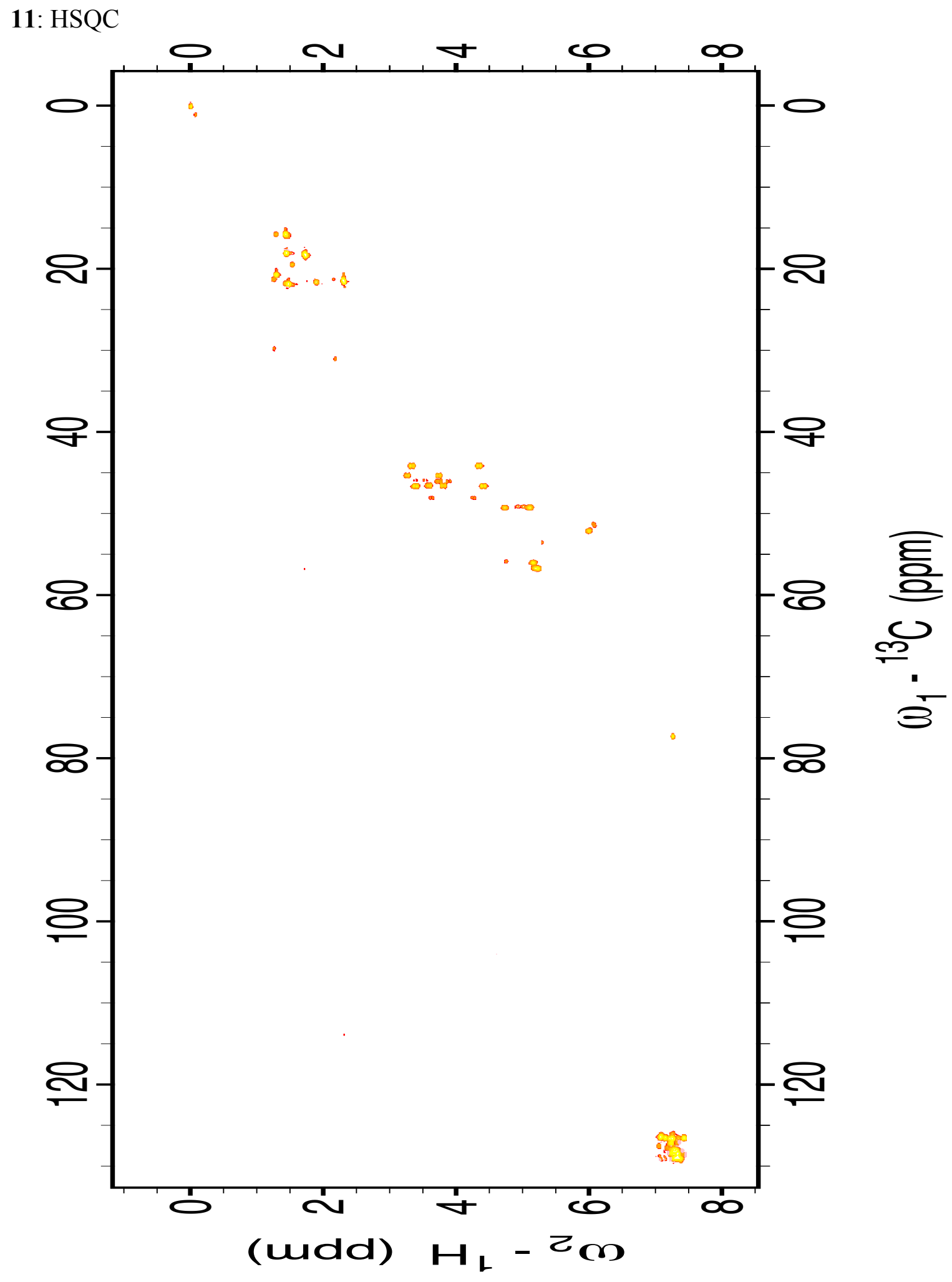




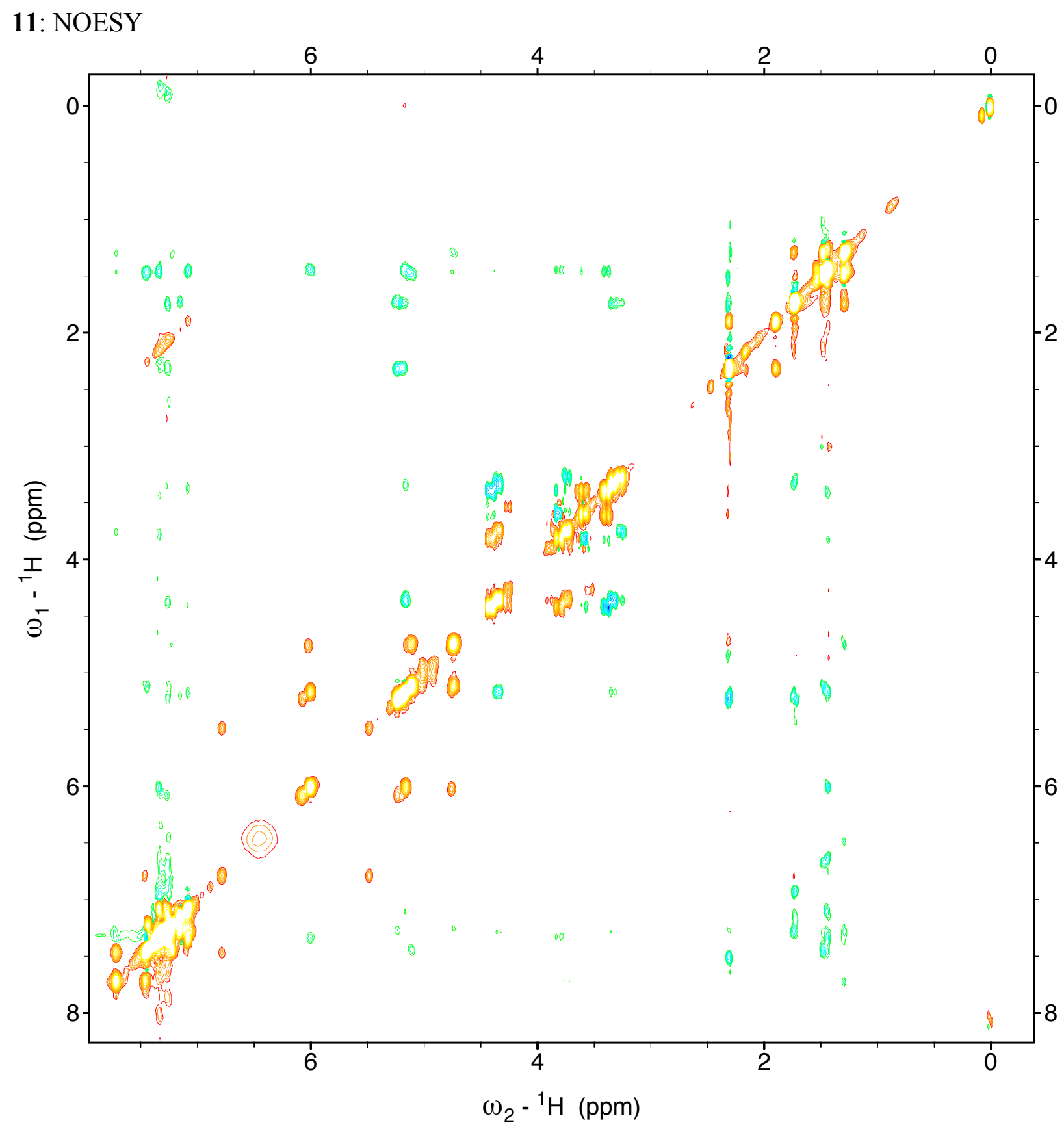

S- 54 


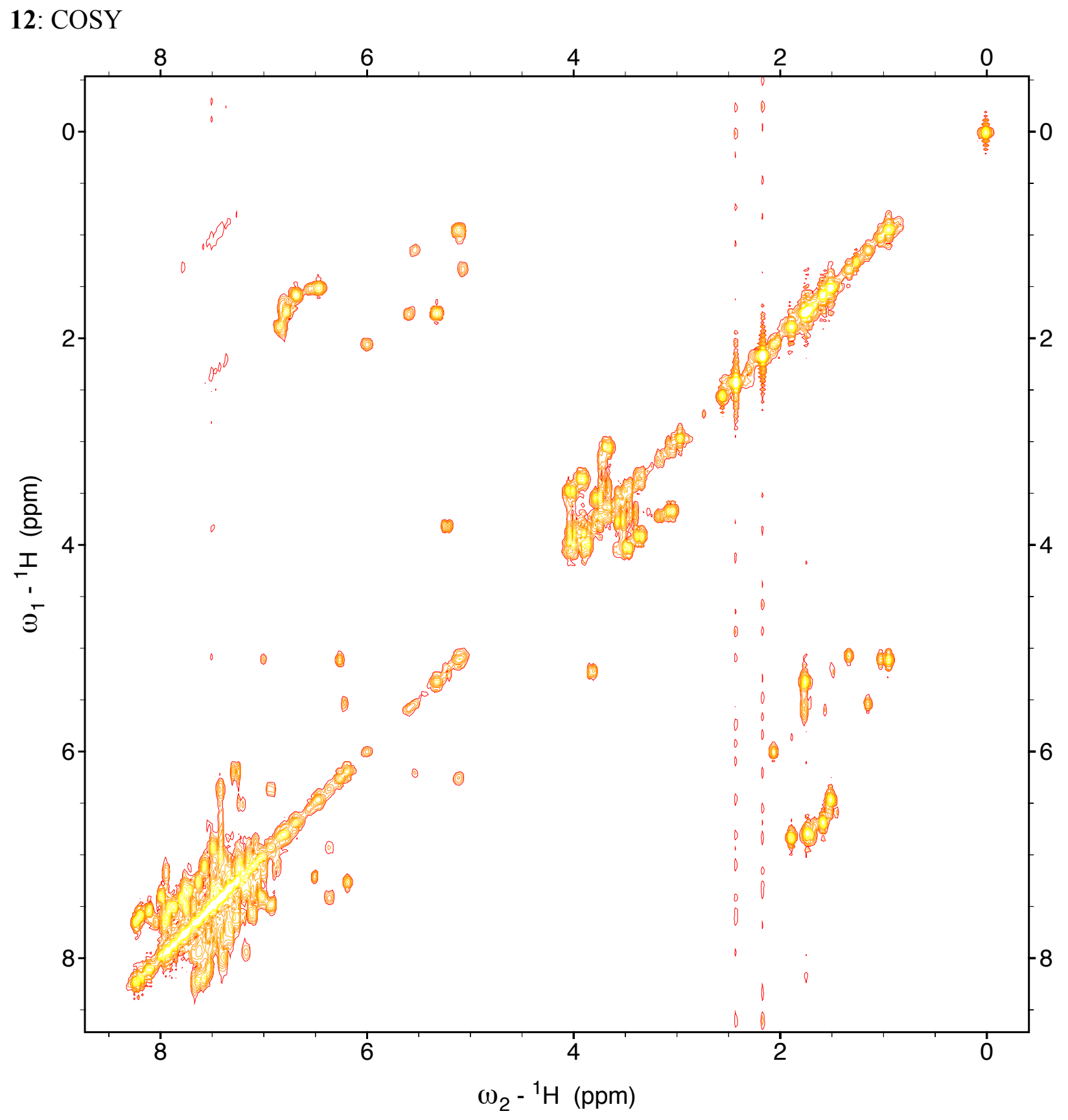

S- 55 
12: HSQC

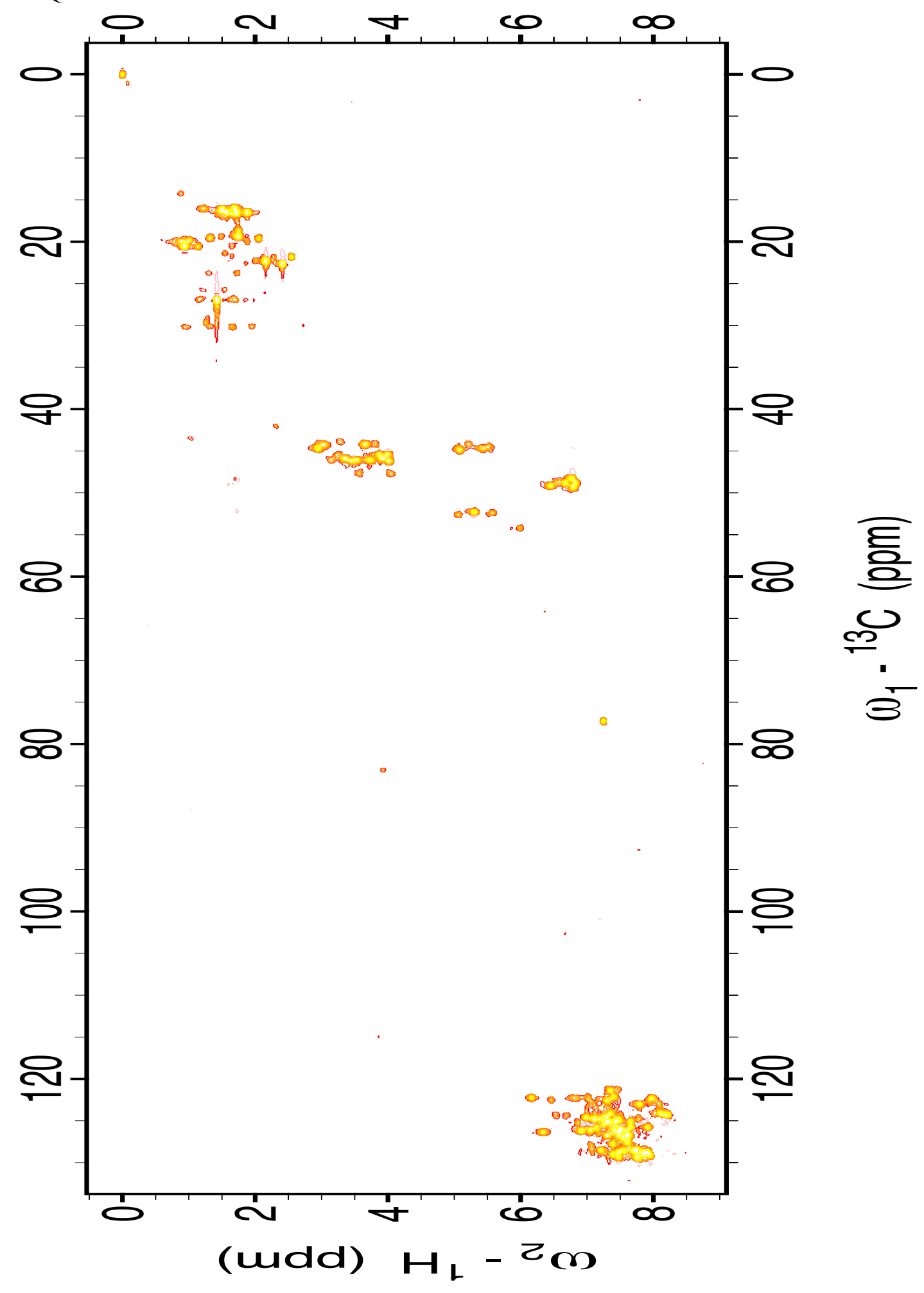




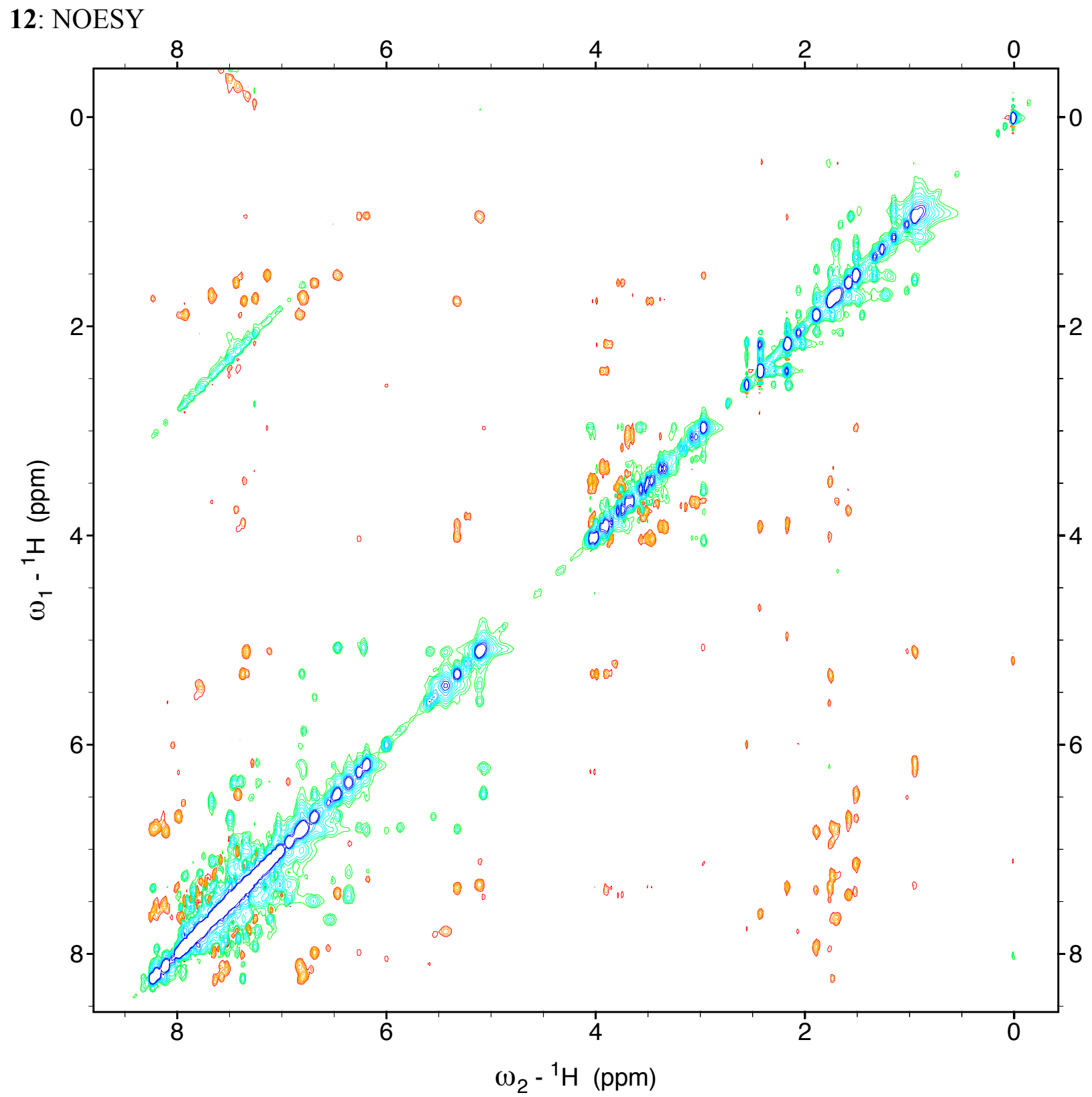

S- 57 


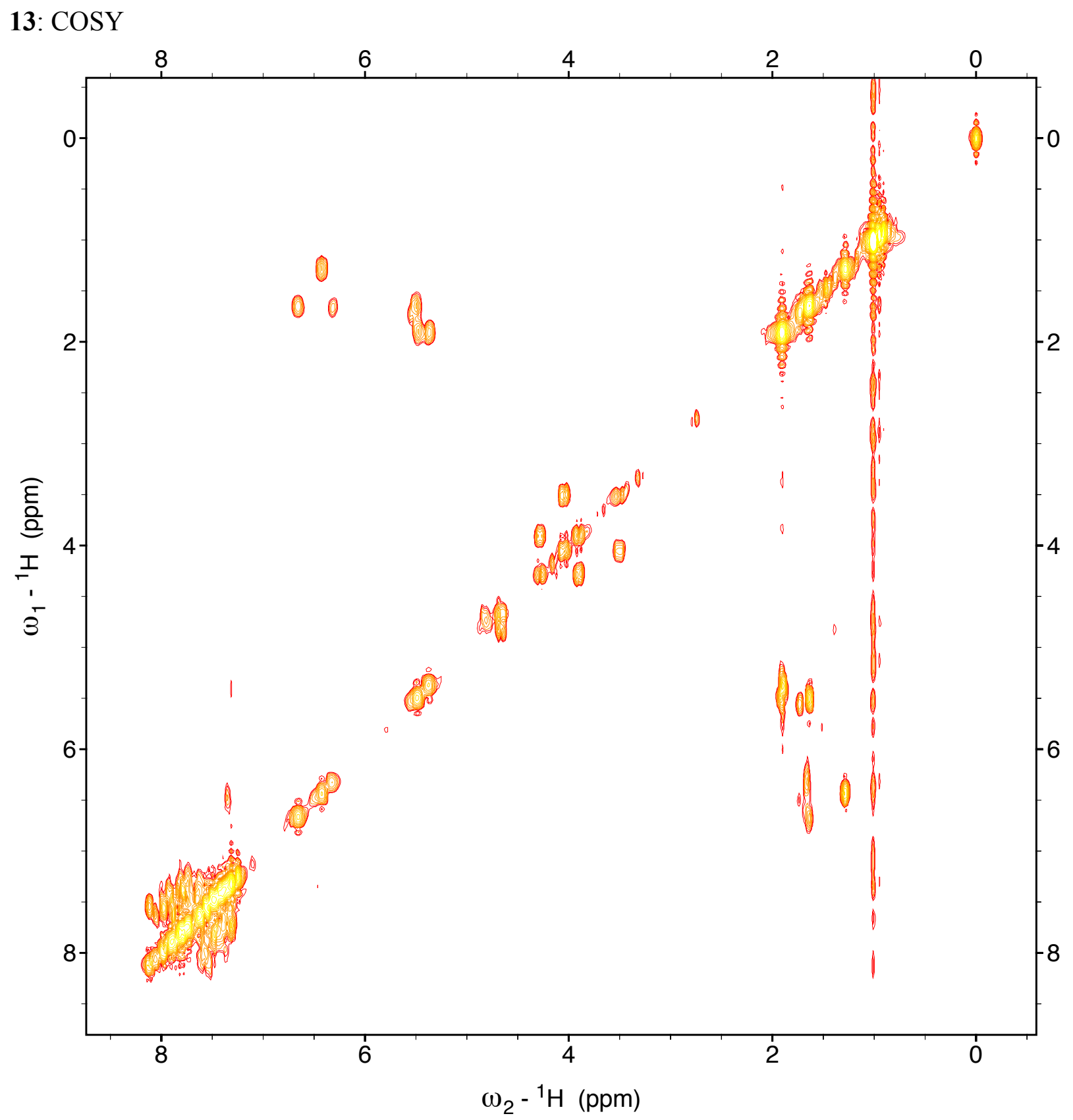

S- 58 


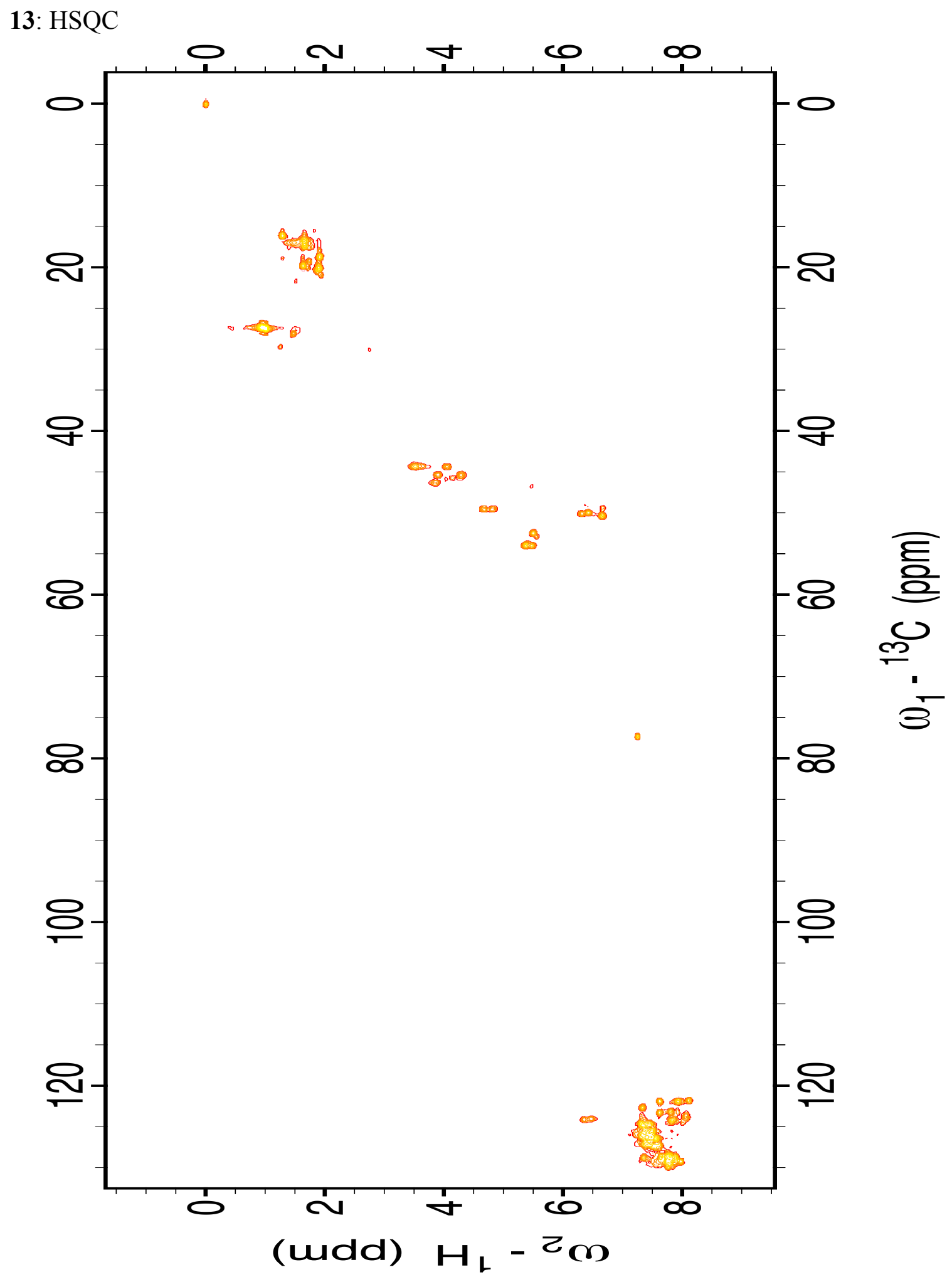




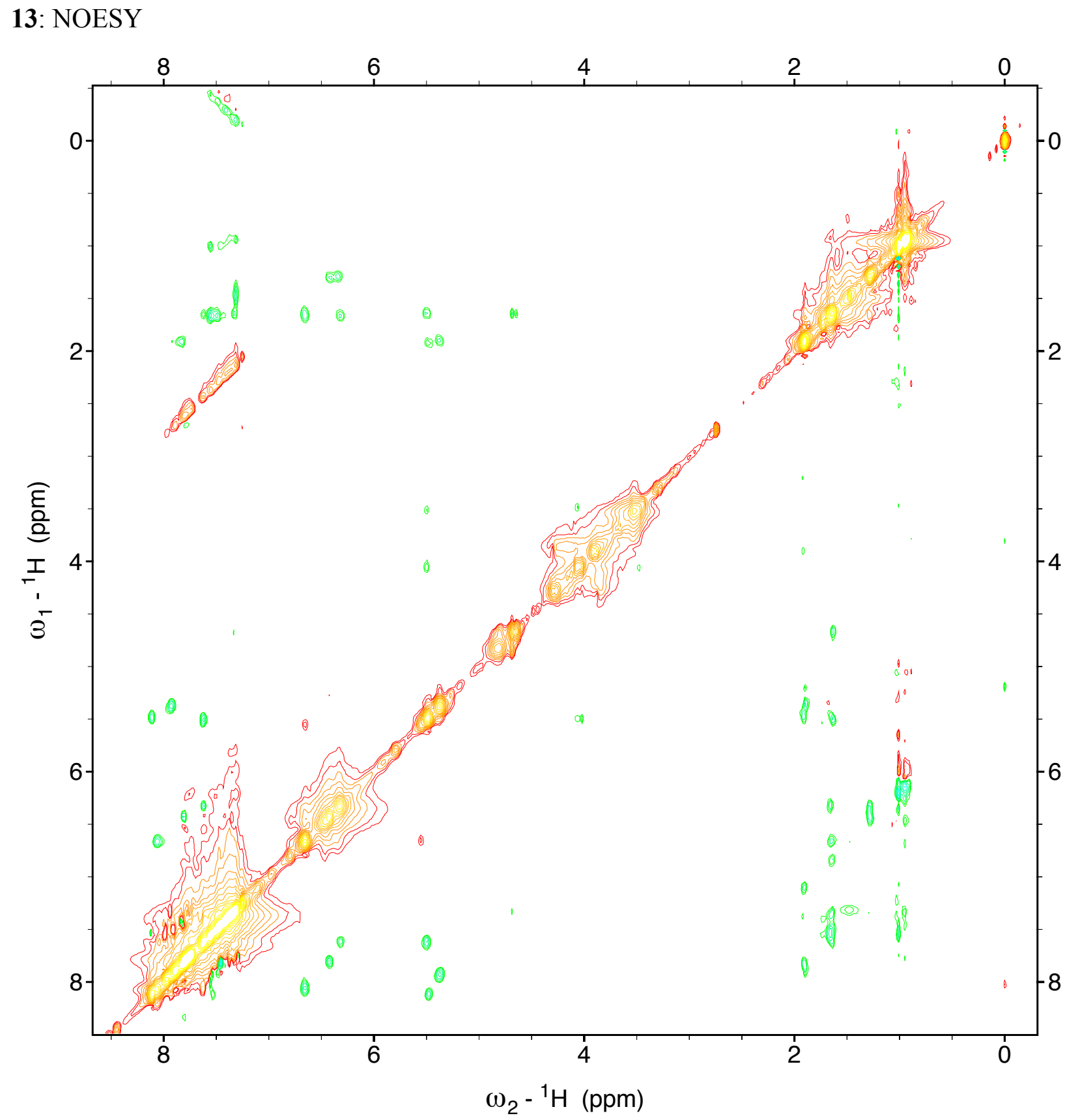




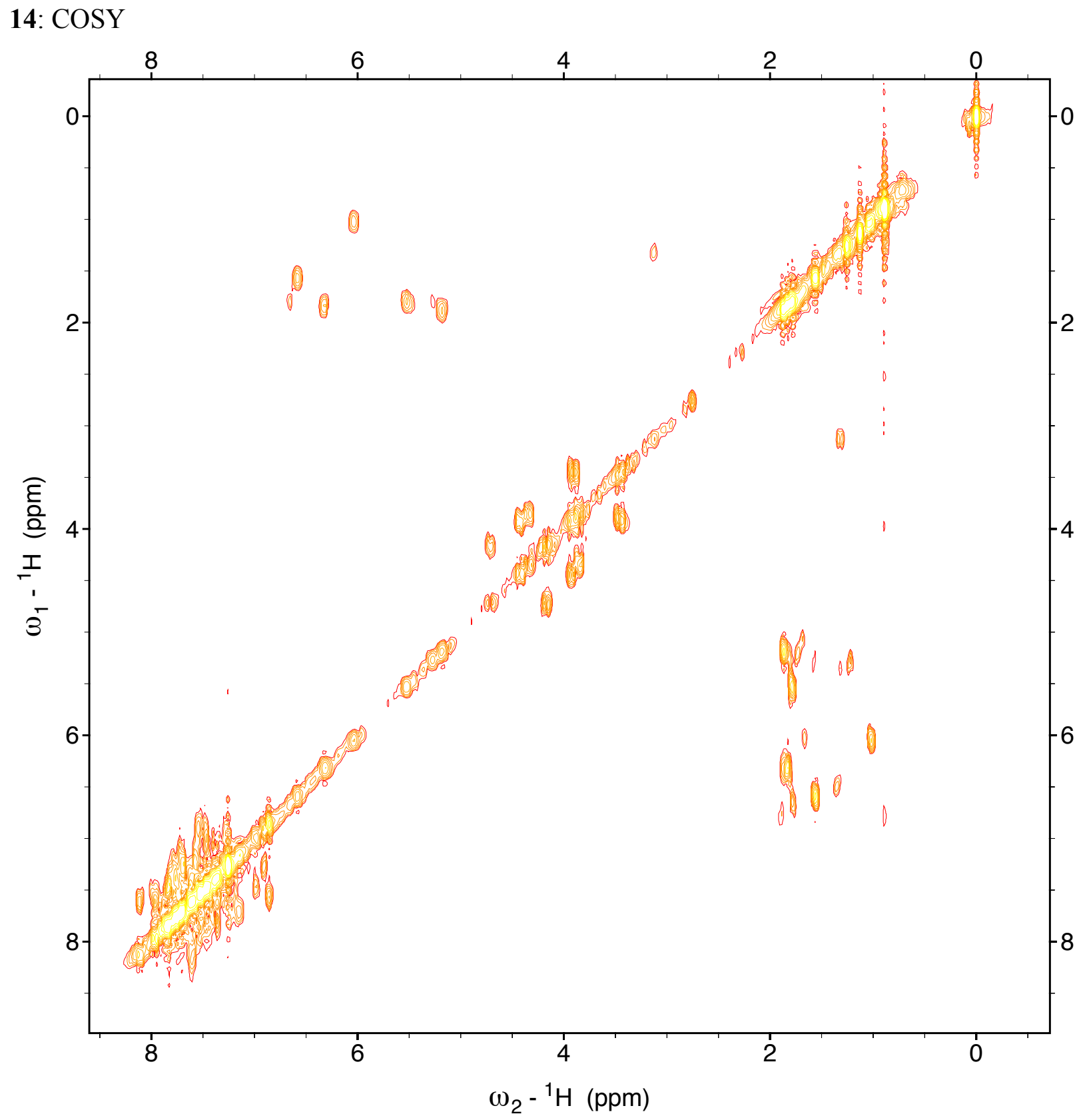

S- 61 


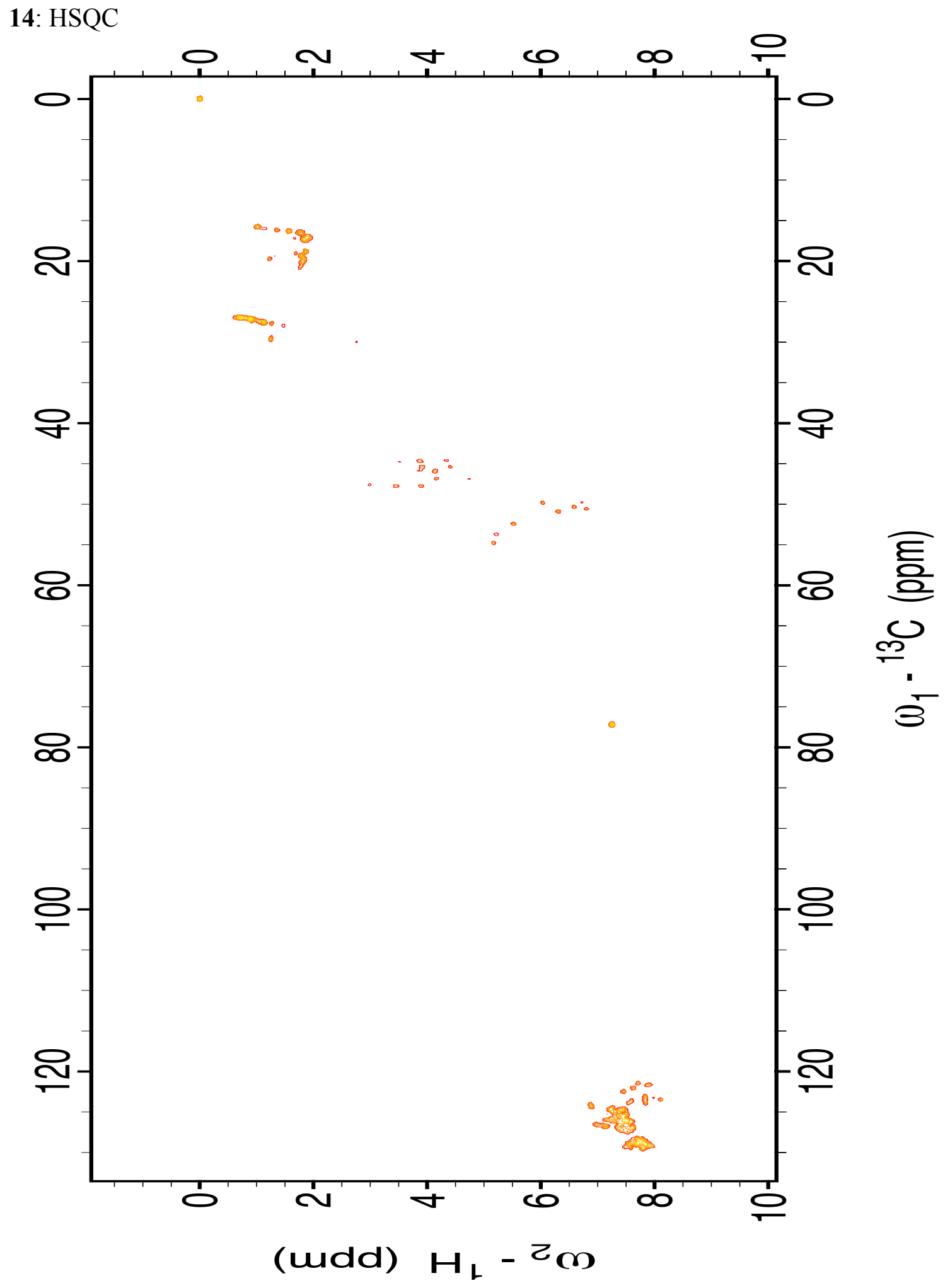




\section{4: NOESY}

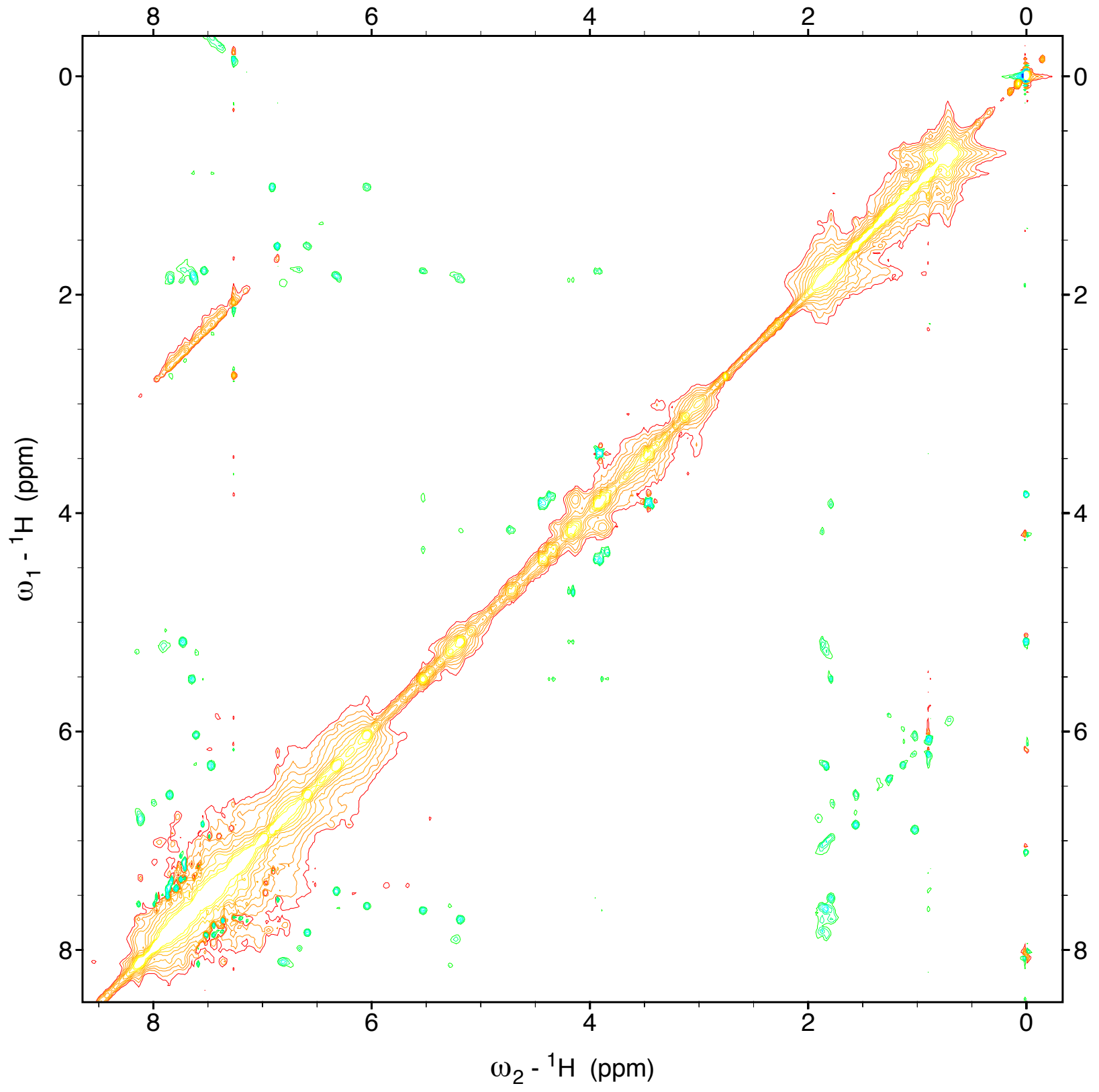


X-ray crystallography. For each structure the selected crystal in Paratone-N oil was maneuvered onto the tip of a glass rod drawn out to a fiber. The X-ray intensity data were measured with phi and omega scans at $173 \mathrm{~K}$ on a Bruker SMART APEX CCD-based X-ray diffractometer system equipped with a Mo-target X-ray tube $(\lambda=0.71073 \AA)$. The detector was placed at a distance of $5.00 \mathrm{~cm}$ from the crystal. A total of 1850 frames were collected (a hemisphere of data). The frames were integrated with the Bruker SAINT software package using a narrow-frame integration algorithm. The structure was solved (direct methods) and refined using the Bruker APEX3 Software Package. Data were corrected for absorption effects using the multi-scan method (SADABS). Further details for each structure can be found in the .cif included as part of the supporting information. Molecular graphics were performed with the UCSF Chimera package. ${ }^{5}$ Chimera is developed by the Resource for Biocomputing, Visualization, and Informatics at the University of California, San Francisco (supported by NIGMS 9P41GM103311). 


\section{References}

(1) T. D. Goddard and D. G. Kneller, SPARKY 3, University of California, San Francisco.

(2) Gorske, B. C.; Jewell, S. A.; Guerard, E. J.; Blackwell, H. E. Org. Lett. 2005, 7 (8), 1521.

(3) Stringer, J. R.; Crapster, J. A.; Guzei, I. A.; Blackwell, H. E. J. Am. Chem. Soc. 2011, 133, 15559.

(4) Wu, C. W.; Kirshenbaum, K.; Sanborn, T. J.; Patch, J. A.; Huang, K.; Dill, K. A.; Zuckermann, R. N.; Barron, A. E. J. Am. Chem. Soc. 2003, 125 (44), 13525.

(5) Pettersen, E. F.; Goddard, T. D.; Huang, C. C.; Couch, G. S.; Greenblatt, D. M.; Meng, E. C.; Ferrin, T. E. J. Comput. Chem. 2004, 25 (13), 1605. 